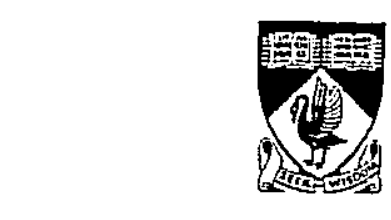

THE MATRIX APPROACH TO

EVALUATING DEMAND

by

Kenneth W. Clements

W. Yang

D. Chen

DISCUSSION PAPER 98.10

DEPARTMENT OF ECONOMICS

THE UNIVERSITY OF WESTERN AUSTRALIA

NEDLANDS, WESTERN AUSTRALIA 6907 


\title{
THE MATRIX APPROACH TO \\ EVALUATING DEMAND
}

\author{
by \\ Kenneth W. Clements \\ W. Yang \\ D. Chen \\ Economic Research Centre \\ Department of Economics \\ The University of Western Australia
}

DISCUSSION PAPER 98.10

DEPARTMENT OF EOCNOMICS

THE UNIVERSITY OF WESTERN AUSTRALIA

NEDLANDS, WESTERN AUSTRALIA 6907

ISSN 0811-6067

ISBN 0-86422-851-1

We would like to acknowledge the financial support of the ARC and the help of Jan Smith. 


\begin{abstract}
As there is a plethora of demand models, which one should be used to estimate income and price elasticities? The paper sheds light on this important practical problem by developing a matrix approach to simulating (MAS) demand equations to analyse their performance under idealised circumstances. Artificial data on the dependent variable are generated by one model, and these are then used for the estimation of another model. Using four popular models, a $4 \times 4$ matrix is generated which gives all pair-wise comparisons. The performance of the models is then evaluated on the basis of the quality of the income and own-price elasticity estimates. The overall results indicate that, among the four models, E. A. Selvanathan's (1985) model performs the best, followed by Working's model under substitution independence (Keller, 1984).
\end{abstract}




\section{TABLE OF CONTENTS}

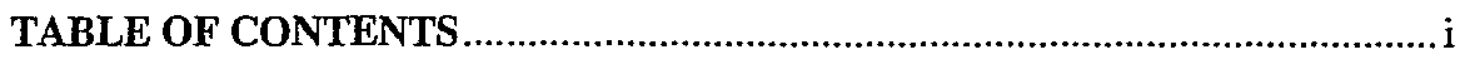

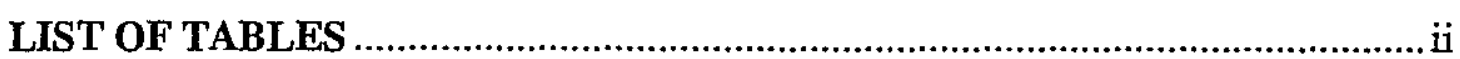

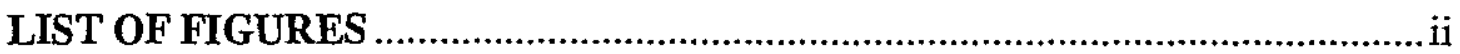

\section{SECTIONS}

1. INTRODUCTION

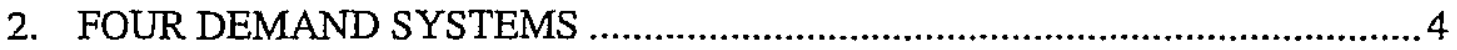

3. THE DATA

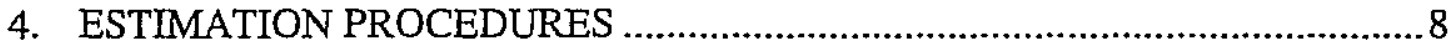

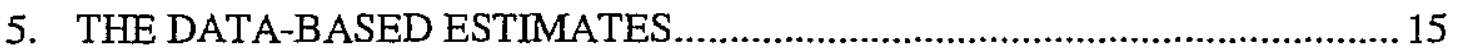

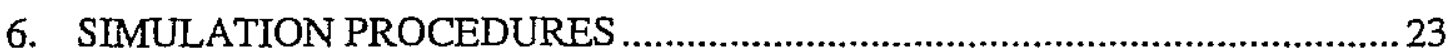

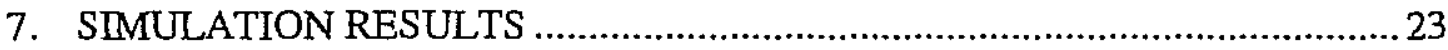

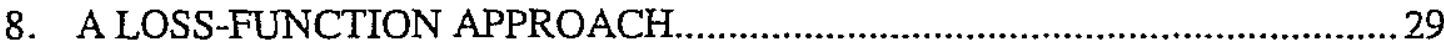

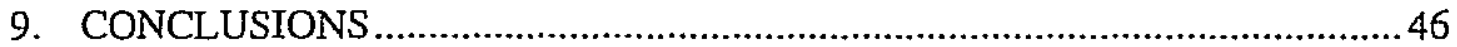

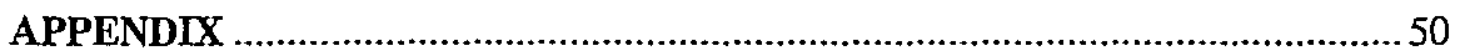

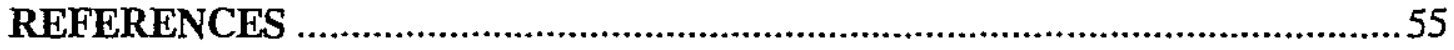




\section{LIST OF TABLES}

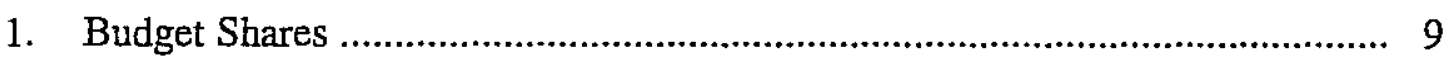

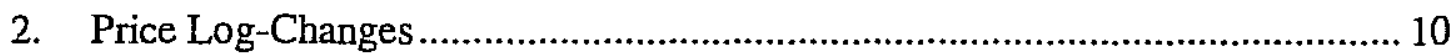

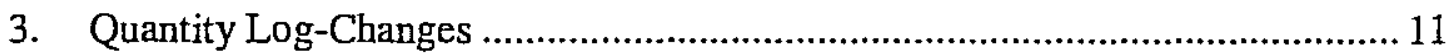

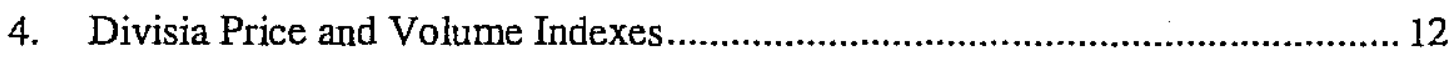

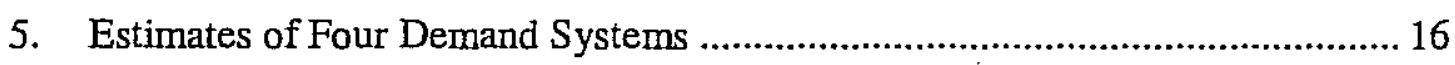

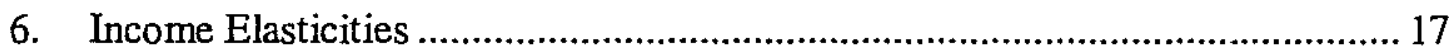

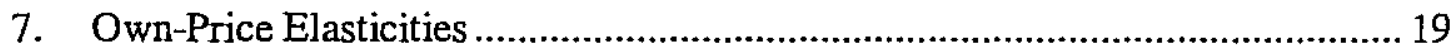

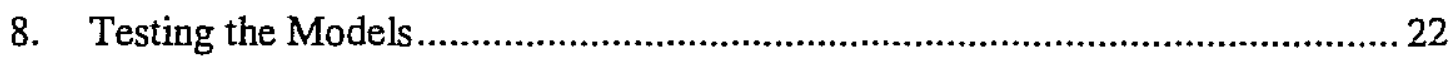

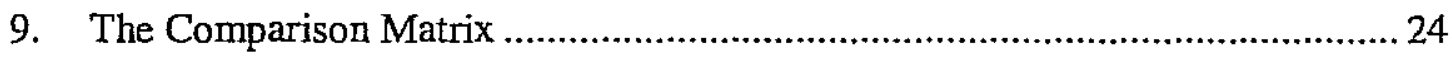

10. Simulation Procedures for $\mathrm{M}(\mathrm{k} \mid \mathrm{j})$.............................................................. 25

11. Biases for Income and Own-Price Elasticities................................................... 30

12. First Summary of Performance of Models .................................................... 32

13. Root-Mean-Square Errors for Income and Own-Price Elasticities...................... 33

14. Standard Errors for Income and Own-Price Elasticities .....................................35

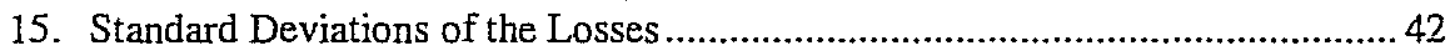

16. Second Summary of Performance of Models .................................................. 43

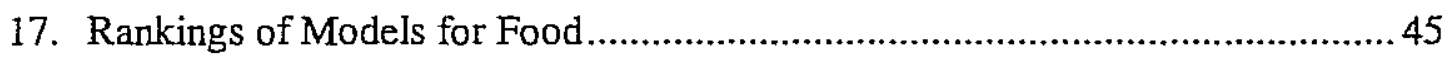

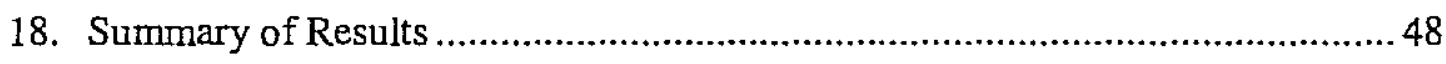

A1. Losses for Income and Own-Price Elasticities When $\alpha=.25 \ldots \ldots \ldots \ldots \ldots \ldots \ldots \ldots \ldots . . .51$

A2. Losses for Income and Own-Price Elasticities When $\alpha=.75 \ldots \ldots \ldots \ldots \ldots \ldots \ldots \ldots \ldots . . . .53$

\section{LIST OF FIGURES}

1. Losses for Income and Own-Price Elasticities ................................................ 38

2. Model Performance ....................................................................................... 44 


\section{INTRODUCTION}

In Australia, beer is much more heavily taxed than wine. A large part of a recent government inquiry into these taxation arrangements (Scales et al., 1995), which was said to have cost over $\$ 1$ million, involved the values of the demand elasticities for beer. There are numerous other instances whereby economists require numerical values of demand elasticities. For example, in industrial organisation, price elasticities are needed to define the size of the market in terms of which goods are closely competitive; complete sets of income and price elasticities are needed as essential inputs in applied general equilibrium models; and computations of optimal taxation and tax reform also use matrices of own- and cross-price elasticities.

How should demand elasticities be estimated? Within the system-wide framework, there are a large number of alternative models which can be used to estimate these elasticities. One way of choosing among these models is the extent to which they satisfy the three major implications of the utility-maximising theory of the consumer. That is, demand functions should conform with the budget constraint that the sum of expenditures on each good be equal to the exogenously-given total expenditure; they should also be homogeneous of degree zero in money income and prices; and the matrix of compensated price slopes should be symmetric negative semi-definite. ${ }^{1}$ But these conditions do not help too much as the number of demand models satisfying these conditions is still quite large.

Another way of proceeding is to use the concept of flexibility of demand models (Diewert, 1971). A demand model is said to be flexible if it is derived from a utility (or expenditure) function which is a second-order approximation to an arbitrary

\footnotetext{
'Strictly speaking, demand functions for an individual consumer should satisfy the last two conditions. Frequently, however, system-wide demand models are applied to aggregate data, notwithstanding the fact that the conditions under which individual demand functions can be aggregated exactly are quite restrictive. An alternative approach to aggregation is the convergence approach which involves the asymptotic behaviour of demand functions as the number of consumers tends to infinity. According to this approach, individual demand functions can be aggregated consistently under much weaker conditions. For details, see Barnett $(1979,1981)$, E. A. Selvanathan $(1991,1995)$ and Theil $(1971$, 1975/76).
} 
function. As Barten (1993) points out, the tendency to make the utility function a quadratic form gives rise to curvature problems over the full range of the exogenous variables. Barten goes on to discuss the empirical comparison of different models using the information inaccuracy, nested and non-nested tests. In the context of differential demand systems, he then proposes choosing between them by taking linear combinations and estimating the weights accorded to each model. He finds that the CBS model (Keller and van Driel, 1985) dominates the Rotterdam (Barten, 1964, Theil, 1965), the almost ideal demand system (Deaton and Muellbauer; 19.80) and Neves' (1994) model.

In this paper we propose an alternative way of choosing between demand models by focusing on their ability to provide satisfactory estimates of income and price elasticities. We do this in the context of a simulation framework in which each of several models has the same probability of being the true model. Each model is used in turn to simulate artificial data and then each model is estimated with these data, yielding a matrix of comparisons which reflects the ability of one model to yield acceptable estimates of the elasticities underlying the model that generated the data. In other words, if $i$ and $j$ denote the true and estimated model and if $n$ is the number of models, then the pairs of indices $i, j=1, \ldots, n$ define the $n^{2}$ cells of a comparison matrix. We christen this procedure the matrix approach to simulating (MAS) demand equations. In principle, MAS could be applied to any goodness-of-fit measure, such as the root-mean-square-error of the system, the information inaccuracy or likelihood value. However, we focus on the quality of the income and price elasticities as their values are frequently the objective of estimating a demand system. An example is the application of the theory of tax reform (Ahmad and Stern, 1984, 1987), in which price elasticities play a prominent role. ${ }^{2}$

The literature related to this paper is quite small. Kiefer and MacKinnon (1976) consider the problem of why estimates of elasticities seem to be heavily dependent on the underlying demand model and propose two possibilities. According

\footnotetext{
2 There is an emerging literature on the sensitivity of the results of tax reform computations to the choice of the demand model. See, e.g.. Decoster and Schokkaert $(1989,1990)$ and Madden (1996).
} 
to their "structuralist explanation", the functional forms of the models estimated simply do not adequately describe the data for whatever reasons. Alternatively, the functional forms, are satisfactory but the stochastic component of the model overwhelms the systematic one, causing imprecise elasticity estimates, which they dub the "stochastic explanation". They consider three demand models, the linear expenditure system (Stone, 1954), the generalised linear expenditure system (Wales, 1971) and the translog (Christensen et al., 1975). Using Monte Carlo simulations with one model generating the data (the "true" model) and another being estimated, Kiefer and MacKinnon examine the quality of the estimated elasticities. If the estimates of the true model are satisfactory, while those of the others are not, the evidence then supports the structuralist explanation and casts doubt on the stochastic explanation. Alternatively, an unsatisfactory performance of the true model (and, presumably, also the others) points to the stochastic explanation. A related paper by Byron (1984) analyses the performance of the Rotterdam model when the data are generated by demand equations associated with a quadratic utility function, the linear expenditure system, the quadratic expenditure system (Howe et al., 1979) and the translog. ${ }^{3}$

Finally, in the context of undersized samples, Chen (1995) uses a given demand system to investigate the performance of four different specifications of the covariance matrix of the error terms of the demand equations. He applies a timeseries version of Working's (1943) model and the covariance matrix is alternatively specified as (i) the ML estimate based on the residuals when the restriction of preference independence is imposed on the model; (ii) proportional to an augmented identity matrix (Deaton, 1975, Theil, 1987); (iii) such that the variances and covariances vary with the relative sizes of the commodities in question, as measured by the budget shares (Barten and Theil, 1964, S. Selvanathan, 1991); and (iv) that proposed by de Boer and Harkema (1986), with and without a bias correction. As Chen's analysis involves simulations revolving around a matrix with rows and

\footnotetext{
${ }^{3}$ See also Wales (1977) who carries out a similar study on the performance of the translog and the generalised Leontief (Diewert, 1971).
} 
columns corresponding to the true and estimated forms of the covariance matrix, this study can also be described as an application of MAS.

This paper is structured as follows. We set out in Section 2 four differential demand models in. which the own-relative price plays a prominent role in the substitution term. Section 3 presents some cross-country data that are used to estimate the models. The estimation procedures and the estimates are given in Sections 4 and 5. Section 6 sets out the application of MAS in considerable detail and the simulation results are presented in Section 7. As the results are expressed in terms of the bias and root-mean-squared error for each elasticity, in Section 8 these are combined into a scalar criterion via a loss function. Concluding comments are given in Section 9.

\section{FOUR DEMAND SYSTEMS}

In this section we present four differential demand systems. The common feature of these models is their simplicity in that each equation contains only the ownrelative price. These systems are not too demanding in terms of data requirements and are straightforward to estimate. Accordingly, these models are frequently used in practice, especially when there is little variability in relative prices and/or in situations when the quality of the data is likely to be low.

Let $p_{i}, q_{i}$ be the price and quantity demanded of good $i$, so that $M=\sum_{i=1}^{n} p_{i} q_{i}$ is total expenditure ("income" for short), where $n$ is the number of goods. Let $w_{i}=p_{i} q_{i} / M$ be the share of income devoted to good $i$, or the $i^{\text {th }}$ budget share. Furthermore, let $\bar{w}_{i}$ be the arithmetic average of $w_{i}$ in years $t$ and $t-1$, and $D p_{i}, D q_{i}$ be the log-change in the price and quantity of $i$ from $t-1$ to 
t. $^{4}$ Finally, define $D Q=\sum_{i=1}^{n} \bar{w}_{i} D_{i}$ as the Divisia volume index and let $y_{i}=\bar{w}_{i}\left(D q_{i}-D Q\right)$.

Working's Model under Substitution Independence

Working's (1943) model under substitution independence (Keller, 1984) takes the following form for good $\mathrm{i}$

$$
y_{i}=\alpha_{i}+\beta_{i} D Q+\lambda\left(\mu_{i}+\bar{w}_{i}\right)\left[D p_{i}-\sum_{j=1}^{n}\left(\mu_{j}+\bar{w}_{j}\right) D p_{j}\right],
$$

where $\alpha_{i}, \beta_{i}, \mu_{i}$ and $\lambda$ are coefficients. Keller (1984) calls equation (2.1) the CBS/SI model. As this equation is formulated in terms of changes, the intercept $\alpha_{i}$ reflects the residual trend in consumption of good $i$. Note that as $\sum_{j}\left(\mu_{j}+\bar{w}_{j}\right)=1$, the second term in the square brackets, $\sum_{j}\left(\mu_{j}+\bar{w}_{j}\right) D_{P_{j}}$, is a weighted-average of the $\mathrm{n}$ prices, or a price index. Accordingly, the whole term in the square brackets is interpreted as the change in the relative price of good $i$. Note also that only the own relative price appears in this equation. The coefficients satisfy the following addingup restrictions: $\quad \sum_{i=1}^{n} \alpha_{i}=0, \quad \sum_{i=1}^{n} \beta_{i}=0, \quad \sum_{i=1}^{n} \mu_{i}=0$. Dividing both sides of equation (2.1) by $\bar{w}_{i}$ and rearranging yields the following income and Slutsky ownprice elasticities for commodity i

$$
\eta_{i}=1+\frac{\beta_{i}}{\bar{w}_{i}}, \quad \eta_{i j}=\frac{\lambda\left(\mu_{i}+\bar{w}_{i}\right)\left[1-\left(\mu_{i}+\bar{w}_{i}\right)\right]}{\bar{w}_{i}} .
$$

\section{Working's Model under Preference Independence}

Working's model under preference independence can be expressed as (see, e.g., Theil and Clements, 1987, Sec. 1.15)

${ }^{4}$ That is, $D p_{i}=\log p_{i t}-\log p_{i, t-1}, D q_{i}=\log q_{i t}-\log q_{i, t-1}$. 


$$
y_{i}=\alpha_{i}+\beta_{i} D Q+\phi\left(\beta_{i}+\bar{w}_{i}\right)\left[D p_{i}-\sum_{j=1}^{n}\left(\beta_{j}+\bar{w}_{j}\right) D p_{j}\right]
$$

where $\phi$ is the income flexibility (the reciprocal of the income elasticity of the marginal utility of income). Keller and van Driel (1985) call equation (2.2) the CBS/PI model. The term in the square brackets is again the change in the $\mathrm{i}^{\text {th }}$ relative price, with a (slightly) different price index acting as the deflator. The adding-up restrictions are $\sum_{i=1}^{n} \alpha_{i}=0, \quad \sum_{i=1}^{n} \beta_{i}=0$. The income and Slutsky own-price elasticities are

$$
\eta_{i}=1+\frac{\beta_{i}}{\bar{w}_{i}}, \quad \eta_{i i}=\frac{\phi\left(\beta_{i}+\bar{w}_{i}\right)\left[1-\left(\beta_{i}+\bar{w}_{i}\right)\right]}{\bar{w}_{i}} .
$$

Note that if the coefficient $\mu_{\mathrm{i}}$ in equation (2.1) equals $\beta_{\mathrm{i}}$, then (2.1) becomes (2.2).

Selvanathan's Model

E. A. Selvanathan's (1985) model can be written as

$$
y_{i}=\alpha_{i}+\beta_{i} D Q+\gamma \bar{w}_{i}\left[D p_{i}-\sum_{j=1}^{n} \bar{w}_{j} D p_{j}\right]
$$

where $\gamma$ is a new coefficient, interpreted as the (common) elasticity of substitution. The adding-up restrictions are $\sum_{i=1}^{n} \alpha_{i}=0, \quad \sum_{i=1}^{n} \beta_{i}=0$. The income and Slutsky own-price elasticities are

$$
\eta_{i}=1+\frac{\beta_{i}}{\bar{w}_{i}}, \quad \eta_{i i}=\gamma\left(1-\bar{w}_{i}\right)
$$

If $\mu_{i}=0$ in equation (2.1), then that model becomes (2.3). 


\section{The Constant Elasticity Model}

The constant elasticity model takes the form

$$
D q_{i}=\alpha_{i}+\eta_{i} D Q+\psi_{i}\left[D p_{i}-\sum_{j=1}^{n} \bar{w}_{j} D p_{j}\right]
$$

where $\eta_{\mathrm{i}}$ is the income elasticity and $\psi_{\mathrm{i}}$ is a new coefficient. The Slutsky own-price elasticity for commodity $i$ is

$$
\eta_{\mathrm{ii}}=\psi_{\mathrm{i}}\left(1-\overline{\mathrm{w}}_{\mathrm{i}}\right)
$$

As is well known, equation (2.4) does not satisfy the budget constraint; but it is still used as a pragmatic model. Note that (2.4) is not a special case of (2.1).

Note that the income parts of models (2.1) to (2.3) are all of the Working (1943) form, which implies that the budget shares are linear functions of the logarithm of income. This functional form is quite popular ${ }^{5}$ and seems to be capable of successfully capturing the curvature of the consumption-income relation in many instances. $^{6}$

\section{THE DATA}

The data used for this study are cross country so that $\overline{\mathrm{w}}_{\mathrm{i}}$ refers to the sample mean over time of the arithmetic average of the budget share for country $c$, to be written as $\bar{w}_{\mathrm{ic}}$. Similarly, $\mathrm{Dp}$ ic and $\mathrm{Dq}_{\mathrm{ic}}$ are the means of the price and quantity log-

\footnotetext{
${ }^{5}$ For example, it underlies the almost ideal model of Deaton and Muellbauer (1980).

${ }^{6}$ For example, Chen (1993, Chapter 5) uses the data employed later in this paper to investigate the functional form of the Engle curve for food for a wide range of countries. On the basis of a Box-Cox analysis, he finds that Working's model dominates.
} 
changes for c. The data are from Chen (1993) with 42 countries and $n=8$ commodities and are presented in Tables 1-3 with countries listed in terms of decreasing GDP per capita. Table 4 contains the Divisia price and volume indexes for each country, $D P_{c}=\sum_{i=1}^{8} \bar{w}_{i c} D p_{i c}, D Q_{c}=\sum_{i=1}^{8} \bar{w}_{i c} D q_{i c}$.

\section{ESTIMATION PROCEDURES}

We apply equation (2.1) to country $c$ and add an error term $\left(\varepsilon_{\text {ic }}\right)$ :

(4.1) $y_{i c}=\alpha_{i}+\beta_{i} D Q_{c}+\lambda\left(\mu_{i}+\bar{w}_{i c}\right)\left[D p_{i c}-\sum_{j=1}^{8}\left(\mu_{j}+\bar{w}_{j c}\right) D p_{j c}\right]+\varepsilon_{i c} \cdot$

The relative price term here is $D p_{i c}-\sum_{j=1}^{8}\left(\mu_{j}+\bar{w}_{j c}\right) D p_{j c}$. As $\sum_{j=1}^{8} \mu_{j}=0$ and $\sum_{j=1}^{8} \bar{w}_{j c}=1$, this can be expressed as $D_{p_{i c}}^{*}-\sum_{j=1}^{7}\left(\mu_{j}+\bar{w}_{j c}\right) D p_{j c}^{*}$, where $\mathrm{DP}_{\mathrm{ic}}^{*}=\mathrm{Dp}_{\mathrm{ic}}-\mathrm{Dp}_{\mathrm{gc}}$ is the change in the price of commodity $\mathrm{i}$ in terms of commodity 8. Accordingly, equation (4.1) can be rewritten as

$$
y_{i c}=\beta_{i} D Q_{c}+\lambda_{z_{i c}}+\alpha_{i}+\varepsilon_{i c}
$$

where $z_{i c}=\left(\mu_{i}+\bar{w}_{i c}\right)\left[D p_{i c}^{*}-\sum_{j=1}^{7}\left(\mu_{j}+\bar{w}_{j c}\right) D p_{j c}^{*}\right]$.

Let $y_{i}=\left[y_{i, 1}, \ldots, y_{i, 42}\right]^{\prime}$ be a vector of the 42 observations on the dependent variable for commodity $i ; \quad X_{i}=\left[\mathbf{D Q}, \mathbf{O}, \mathbf{Z}_{\mathbf{i}}, 1\right]$ be a $42 \times 4$ matrix, with $\mathbf{D Q}=\left[\mathrm{DQ}_{1}, \ldots, \mathrm{DQ}_{42}\right]^{\prime}, \mathbf{O}$ a vector of zeros, $\mathrm{Z}_{\mathrm{i}}=\left[\mathrm{z}_{\mathrm{i}, 1}, \ldots, \mathrm{z}_{\mathrm{i}, 42}\right]^{\prime}$ and $\mathrm{i}$ a 
TABLE 1

BUDGET SHARES

\begin{tabular}{|c|c|c|c|c|c|c|c|c|}
\hline $\begin{array}{c}\text { Country } \\
\text { (1) }\end{array}$ & $\begin{array}{l}\text { Food } \\
\text { (2) }\end{array}$ & $\begin{array}{c}\text { Clothing } \\
\text { (3) }\end{array}$ & $\begin{array}{c}\text { Housing } \\
\text { (4) }\end{array}$ & $\begin{array}{c}\text { Durables } \\
\text { (5) }\end{array}$ & $\begin{array}{c}\text { Medicine } \\
\text { (6) }\end{array}$ & $\begin{array}{c}\text { Transport } \\
\text { (7) }\end{array}$ & $\begin{array}{c}\text { Recreation } \\
\text { (8) }\end{array}$ & $\begin{array}{l}\text { Other } \\
\text { (9) }\end{array}$ \\
\hline 1.USA & 17.6 & 7.6 & 19.3 & 6.9 & 10.6 & 16.4 & 8.4 & 13.2 \\
\hline 2. Switzerland & 32.2 & 6.8 & 18.1 & 7.8 & 6.5 & 11.0 & 9.4 & 8.3 \\
\hline 3. Canada & 22.6 & 8.4 & 18.9 & 8.7 & 3.8 & 15.0 & 8.3 & 14.2 \\
\hline 4. USA* & 26.7 & 9.5 & 22.7 & 7.3 & 8.1 & 15.2 & 5.5 & 4.9 \\
\hline 5. Australia & 26.9 & 8.5 & 16.6 & 8.3 & 6.2 & 15.5 & 6.8 & 11.1 \\
\hline 6. Sweden & 27.8 & 8.0 & 23.4 & 7.7 & 2.3 & 13.9 & 9.8 & 7.0 \\
\hline 7. Denmark & 28.3 & 7.0 & 20.9 & 9.0 & 1.9 & 15.0 & 8.7 & 9.2 \\
\hline 8. France & 25.9 & 8.2 & 14.4 & 10.2 & 10.5 & 12.0 & 6.3 & 12.4 \\
\hline 9. Norway & 31.0 & 10.0 & 14.5 & 8.9 & 42 & 13.3 & 8.2 & 9.9 \\
\hline 10. UK & 24.1 & 8.1 & 18.8 & 7.6 & .8 & 13.6 & 10.2 & 16.6 \\
\hline 11. Germany & 30.1 & 10.4 & 15.8 & 12.0 & 2.7 & 13.1 & 7.4 & 8.5 \\
\hline 12. Japan & 27.4 & 7.8 & 16.8 & 6.8 & 8.9 & 9.0 & 9.1 & 14.3 \\
\hline 13. Israel & 29.2 & 6.8 & 22.1 & 12.5 & 3.5 & 11.1 & 5.6 & 9.0 \\
\hline 14. Italy & 35.2 & 9.4 & 13.0 & 7.0 & 3.9 & 11.0 & 7.4 & 13.1 \\
\hline 15. Belgium & 30.1 & 7.5 & 15.7 & 14.1 & 6.7 & 10.7 & 4.2 & 10.9 \\
\hline 16. Austria & 29.3 & 11.4 & 12.0 & 8.9 & 3.5 & 13.4 & 6.0 & 15.5 \\
\hline 17. Australia* & 33.3 & 11.0 & 12.7 & 7.8 & 5.7 & 13.1 & 4.3 & 12.1 \\
\hline 18. Hong Kong & 29.1 & 18.8 & 14.4 & 10.1 & 5.4 & 75 & 8.1 & 6.6 \\
\hline 19. Sweden* & 36.6 & 11.5 & 15.9 & 7.0 & 3.7 & 14.2 & 8.9 & 2.4 \\
\hline 20. Netherlands & 32.6 & 14.8 & 10.8 & 125 & 6.7 & 6.7 & 6.5 & 9.5 \\
\hline 21. Finland & 36.9 & 8.3 & 14.4 & 7.5 & 2.5 & 13.6 & 7.4 & 9.5 \\
\hline 22. Iceland & 31.4 & 10.7 & 19.1 & 10.0 & 6.1 & 12.1 & 5.7 & 5.0 \\
\hline 23. $\mathrm{UK}^{*}$ & 39.7 & 10.7 & 18.3 & 6.6 & 2.3 & 10.9 & 7.5 & 4.0 \\
\hline 24. Singapore & 28.7 & 8.9 & 9.6 & 8.5 & 2.7 & 12.4 & 11.0 & 18.3 \\
\hline 25. Spain & 37.6 & 10.6 & 14.0 & 8.5 & 4.6 & 9.5 & 6.3 & 8.9 \\
\hline 26. Mexico & 38.9 & 11.2 & 9.9 & 12.2 & 3.6 & 9.0 & 5.0 & 10.1 \\
\hline 27. Israel & 31.9 & 9.4 & 19.2 & 7.4 & 6.6 & 7.3 & 8.1 & 10.0 \\
\hline 28. Italy* & 46.3 & 10.1 & 16.6 & 3.2 & 6.3 & 8.1 & 75 & 1.8 \\
\hline 29. Puerto Rico & 31.0 & 9.9 & 14.0 & 8.4 & 4.4 & 14.8 & 6.6 & 10.8 \\
\hline 30. Malta & 36.0 & 8.7 & 6.8 & 10.9 & 3.9 & 13.9 & 6.5 & 13.2 \\
\hline 31. Puerto Rico* & 35.6 & 10.6 & 15.3 & 6.9 & 6.9 & 12.3 & 8.8 & 3.7 \\
\hline 32. Ireland & 49.2 & 10.0 & 12.8 & 5.7 & 1.3 & 8.8 & 6.3 & 5.9 \\
\hline 33. South Africa & 36.8 & 11.8 & 16.7 & 7.8 & 4.8 & 13.2 & 4.7 & 4.1 \\
\hline 34. Colombia & 39.4 & 7.6 & 12.4 & 5.7 & 5.4 & 12.7 & 5.4 & 11.5 \\
\hline 35. Taiwan & 48.5 & 5.3 & 16.2 & 4.8 & 4.6 & 3.9 & 9.5 & 7.1 \\
\hline 36. Korea & 50.8 & 8.6 & 8.9 & 4.7 & 3.2 & 8.3 & 7.3 & 8.3 \\
\hline 37. Greece & 46.8 & 12.2 & 18.3 & 3.9 & 3.6 & 6.9 & 6.1 & 2.3 \\
\hline 38. Panama & 455 & 7.4 & 16.7 & 6.4 & 4.7 & 9.4 & 7.5 & 2.3 \\
\hline 39. Thailand & 52.6 & 9.5 & 6.8 & 5.7 & 5.0 & 9.1 & 6.0 & 5.2 \\
\hline 40. Sri Lanka & 64.2 & 65 & 6.4 & 4.4 & 2.2 & 9.0 & 4.8 & 2.4 \\
\hline 41. Thailand* & 57.6 & 8.4 & 8.0 & 3.2 & 5.6 & 7.9 & 7.5 & 1.8 \\
\hline 42. Korea* & 59.9 & 10.4 & 11.4 & 2.8 & 4.2 & 4.7 & 4.4 & 2.2 \\
\hline Mean & 36.2 & 9.5 & 15.0 & 7.8 & 4.8 & 11.2 & 7.1 & 8.5 \\
\hline
\end{tabular}

Note: An asterisk $\left(^{*}\right)$ indicates a second period for a country. All entries are to be divided by 100 .

Source: Chen (1993) 
TABLE 2

PRICE LOG-CHANGES

\begin{tabular}{|c|c|c|c|c|c|c|c|c|}
\hline $\begin{array}{c}\text { Country } \\
\text { (1) }\end{array}$ & $\begin{array}{l}\text { Food } \\
\text { (2) }\end{array}$ & $\begin{array}{c}\text { Clothing } \\
\text { (3) }\end{array}$ & $\begin{array}{c}\text { Housing } \\
\text { (4) }\end{array}$ & $\begin{array}{c}\text { Durabies } \\
\text { (5) }\end{array}$ & $\begin{array}{c}\text { Medicine } \\
\text { (6) }\end{array}$ & $\begin{array}{c}\text { Transport } \\
\text { (7) }\end{array}$ & $\begin{array}{c}\text { Recreation } \\
\text { (8) }\end{array}$ & $\begin{array}{l}\text { Other } \\
\text { (9) }\end{array}$ \\
\hline 1. USA & 6.79 & 3.80 & 6.64 & 5.85 & 7.45 & 6.88 & 5.06 & 7.15 \\
\hline 2. Switzerland & 4.02 & 3.39 & 5.43 & 3.86 & 6.49 & 4.55 & 4.10 & 5.47 \\
\hline 3. Canada & 5.71 & 3.87 & 5.18 & 4.49 & 4.96 & 4.72 & 4.92 & 6.73 \\
\hline 4. USA* & 1.85 & 1.62 & 1.70 & 55 & 3.04 & 1.52 & 2.35 & 4.31 \\
\hline 5. Australia & 7.95 & 8.12 & 8.89 & 6.23 & 10.41 & 8.40 & 8.78 & 9.40 \\
\hline 6. Sweden & 8.14 & 5.52 & 9.30 & 8.28 & 6.78 & 8.60 & 7.37 & 9.95 \\
\hline 7. Denmark & 8.09 & 6.57 & 11.43 & 8.55 & 8.91 & 8.79 & 7.78 & 9.30 \\
\hline 8. France & 7.15 & 6.74 & 8.80 & 6.76 & 6.99 & 8.13 & 6.25 & 8.28 \\
\hline 9. Norway & 6.96 & 6.74 & 7.20 & 7.02 & 8.25 & 7.32 & 5.55 & 7.91 \\
\hline 10. UK & 10.65 & 8.39 & 13.04 & 10.35 & 11.34 & 12.22 & 10.76 & 11.56 \\
\hline 11. Germany & 3223 & 3.67 & 5.57 & 3.04 & 5.58 & 4.13 & 3.74 & 4.61 \\
\hline 12. Japan & 7.61 & 7.99 & 7.58 & 6.71 & 7.36 & 8.70 & 7.35 & 8.88 \\
\hline 13. Israel & 54.06 & 50.21 & 54.70 & 50.42 & 54.87 & 53.40 & 54.87 & 54.72 \\
\hline 14. Italy & 11.42 & 12.88 & 12.88 & 13.24 & 10.42 & 13.32 & 11.16 & 14.21 \\
\hline 15. Belgium & 4.59 & 3.89 & 5.76 & 4.36 & 6.02 & 5.28 & 4.97 & 6.88 \\
\hline 16. Austria & 4.18 & 3.67 & 7.49 & 3.59 & 8.94 & 6.12 & 4.18 & 6.53 \\
\hline 17. Australia* & 2.32 & 1.39 & 4.87 & .48 & 3.15 & 1.96 & 2.92 & 3.02 \\
\hline 18. Hong Kong & 8.62 & 8.99 & 8.51 & 6.53 & 9.54 & 9.61 & 8.33 & 10.40 \\
\hline 19. Sweden* & 4.00 & 2.25 & 3.85 & 1.79 & 2.85 & 3.46 & 4.22 & 3.70 \\
\hline 20. Netherlands & 3.50 & 3.48 & 5.08 & 2.29 & 7.73 & 3.38 & 5.71 & 4.80 \\
\hline 21. Finland & 7.46 & 6.09 & 5.90 & 6.25 & 6.22 & 7.16 & 6.83 & 8.17 \\
\hline 22. Iceland & 13.23 & 9.69 & 11.66 & 10.13 & 14.10 & 11.25 & 12.66 & 11.48 \\
\hline 23. UK* & 2.11 & 1.70 & 4.68 & 1.69 & 3.09 & 2.93 & 4.62 & 3.80 \\
\hline 24. Singapore & 4.38 & 2.85 & 4.18 & 4.01 & 5.22 & 3.85 & 1.19 & 3.90 \\
\hline 25. Spain & 959 & 11.24 & 8.58 & 11.07 & 11.69 & 7.89 & 12.62 & 10.87 \\
\hline 26. Mexico & 21.38 & 22.34 & 19.62 & 22.60 & 22.11 & 23.97 & 22.14 & 23.99 \\
\hline 27. Israel & 4.85 & 4.65 & 7.81 & 1.94 & 6.46 & 8.06 & 5.97 & 7.22 \\
\hline 28. Italy* & 2.52 & 2.17 & 3.65 & -.16 & 4.24 & 2.27 & 4.07 & 4.19 \\
\hline 29. Puerto Rico & 6.95 & 3.97 & 6.20 & 4.39 & 6.46 & 5.61 & 3.67 & 5.46 \\
\hline 30. Malta & 5.81 & 1.10 & 1.98 & 4.71 & 4.07 & 8.27 & 4.42 & 6.60 \\
\hline 31. Puerto Rico* & 2.62 & 1.44 & 1.27 & .83 & 5.76 & 2.38 & 3.20 & 2.46 \\
\hline 32. Ireland & 3.18 & .89 & 3.98 & 3.15 & 1.33 & 3.13 & 3.63 & 4.24 \\
\hline 33. South Africa & 2.27 & .20 & 350 & 32 & 3.90 & 2.40 & 2.49 & 3.40 \\
\hline 34. Colombia & 20.80 & 20.73 & 21.74 & 19.78 & 22.01 & 20.97 & 21.10 & 21.78 \\
\hline 35. Taiwan & 6.03 & 4.07 & 5.75 & 7.02 & 6.16 & 5.01 & 7.05 & 6.44 \\
\hline 36. Korea & 15.38 & 13.02 & 17.71 & 15.08 & 11.33 & 16.28 & 19.92 & 1758 \\
\hline 37. Greece & 2.57 & 1.20 & 1.59 & .67 & 2.48 & 1.87 & 55 & .69 \\
\hline 38. Panama & 1.95 & .59 & 54 & .11 & 2.24 & .04 & .97 & .00 \\
\hline 39. Thailand & 7.46 & 9.42 & 7.52 & 7.15 & 6.44 & 9.49 & 6.12 & 6.43 \\
\hline 40. Sri Lanka & 8.85 & 2.83 & 6.07 & 7.51 & 6.62 & 9.82 & 8.23 & 8.39 \\
\hline 41. Thailand* & 3.24 & .19 & -2.63 & -1.64 & 1.33 & .06 & 2.43 & 2.02 \\
\hline 42. Korea* & 13.28 & 14.75 & 11.27 & 12.54 & 16.70 & 12.69 & 13.91 & 14.80 \\
\hline Mean & 8.02 & 6.86 & 8.25 & 6.99 & 8.60 & 8.24 & 8.05 & 8.85 \\
\hline
\end{tabular}

See note to Table 1.

Source: Chen (1993). 
TABLE 3

QUANTITY LOG-CHANGES

\begin{tabular}{|c|c|c|c|c|c|c|c|c|}
\hline $\begin{array}{c}\text { Country } \\
\text { (1) }\end{array}$ & $\begin{array}{l}\text { Food } \\
\text { (2) }\end{array}$ & $\begin{array}{c}\text { Clothing } \\
\text { (3) }\end{array}$ & $\begin{array}{l}\text { Housing } \\
\text { (4) }\end{array}$ & $\begin{array}{c}\text { Durables } \\
\text { (5) }\end{array}$ & $\begin{array}{c}\text { Medicine } \\
\text { (6) }\end{array}$ & $\begin{array}{c}\text { Transport } \\
\text { (7) }\end{array}$ & $\begin{array}{l}\text { Recreation } \\
\text { (8) }\end{array}$ & $\begin{array}{l}\text { Other } \\
\text { (9) }\end{array}$ \\
\hline 1. USA & .59 & 2.55 & 2.81 & .61 & 4.31 & 2.06 & 3.21 & 1.13 \\
\hline 2. Switzerland & 1.68 & .75 & 2.08 & .62 & 2.85 & 3.63 & 2.98 & 2.79 \\
\hline 3. Canada & 1.41 & 3.12 & 3.17 & 3.02 & .22 & 4.17 & 5.83 & 2.79 \\
\hline 4. USA* & .99 & 2.42 & 2.79 & 3.70 & 3.90 & 2.78 & 2.86 & 3.63 \\
\hline 5. Australia & 1.24 & .49 & 3.74 & 5.04 & 1.22 & 2.92 & 2.25 & 1.77 \\
\hline 6. Sweden & .22 & 3.30 & 2.31 & 1.06 & 2.95 & 1.64 & 4.38 & -1.41 \\
\hline 7. Denmark & 54 & .59 & 3.23 & -1.01 & 1.50 & 1.49 & 3.87 & .75 \\
\hline 8. France & 1.57 & 1.71 & 4.89 & 3.31 & 6.68 & 4.65 & 5.24 & 3.20 \\
\hline 9. Norway & 1.51 & 1.40 & 3.43 & 3.21 & 2.14 & 4.06 & 5.24 & 2.17 \\
\hline 10. UK & .53 & 2.67 & 1.63 & 1.70 & 1.50 & 2.44 & 3.20 & 1.90 \\
\hline 11. Germany & 2.02 & 2.36 & 3.24 & 3.51 & 1.45 & 5.69 & 3.90 & 3.77 \\
\hline 12. Japan & 150 & 1.47 & 4.93 & 1.58 & 6.07 & 4.10 & 3.26 & 3.40 \\
\hline 13. Israel & 1.42 & .49 & 3.50 & 4.06 & 4.04 & 4.83 & 1.40 & 2.31 \\
\hline 14. Italy & 1.78 & 2.16 & 2.46 & 3.76 & 6.43 & 4.53 & 3.89 & 3.15 \\
\hline 15. Belgium & 1.76 & 2.76 & 2.80 & 4.20 & 5.13 & 4.52 & 3.92 & 2.98 \\
\hline 16. Austria & 2.00 & 4.26 & 4.29 & 3.61 & 2.63 & 5.58 & 4.22 & 1.98 \\
\hline 17. Australia* & .78 & .66 & 2.34 & 3.14 & 4.09 & 3.12 & .10 & 1.89 \\
\hline 18. Hong Kong & 2.04 & 591 & 6.36 & 10.26 & 6.26 & 4.55 & 5.97 & 7.41 \\
\hline 19. Sweden* & 151 & 1.76 & 2.77 & 5.25 & 5.62 & 5.32 & 2.28 & 4.03 \\
\hline 20. Netherlands & 2.69 & 3.19 & 3.93 & 6.91 & 5.53 & 752 & 4.54 & 5.20 \\
\hline 21. Finland & 2.54 & 1.62 & 3.60 & 5.41 & 6.43 & 6.48 & 5.03 & 5.58 \\
\hline 22. Iceland & 2.42 & 5.65 & 2.54 & 8.12 & 7.20 & 8.59 & 5.84 & 856 \\
\hline 23. $\mathrm{UK}^{*}$ & 1.62 & 2.44 & 1.98 & 3.08 & 3.75 & 5.53 & 1.37 & 6.48 \\
\hline 24. Singapore & 3.07 & 5.32 & 5.62 & 5.69 & 5.77 & 6.88 & 9.72 & 6.72 \\
\hline 25. Spain & 2.93 & 3.00 & 3.79 & 3.44 & 9.24 & 10.66 & 4.97 & 5.73 \\
\hline 26. Mexico & 1.50 & 1.17 & 1.09 & 1.30 & 5.84 & 3.33 & 2.13 & 1.81 \\
\hline 27. Istael & 4.62 & 6.87 & 5.41 & 11.67 & 5.96 & 9.24 & 8.58 & 5.18 \\
\hline 28. Italy* & 4.09 & 4.62 & 4.62 & 8.51 & 5.96 & 9.46 & 4.28 & 4.47 \\
\hline 29. Puerto Rico & -.14 & 3.03 & 3.37 & .93 & 4.00 & 3.66 & 5.47 & 3.11 \\
\hline 30. Malta & 2.16 & 2.42 & 4.45 & 1.47 & 3.10 & .88 & 2.59 & 8.43 \\
\hline 31. Puerto Rico* & -.28 & 3.91 & 3.25 & 4.45 & 1.40 & 5.13 & 4.89 & 5.89 \\
\hline 32. Ireland & 1.91 & 4.88 & 2.51 & 5.85 & 2.71 & 5.32 & 3.72 & 2.47 \\
\hline 33. South Africa & 1.04 & 2.20 & .57 & 3.21 & 1.81 & 3.32 & 1.22 & 2.48 \\
\hline 34. Colombia & 1.55 & -1.05 & 1.96 & 1.72 & 1.36 & 4.21 & 1.10 & 3.52 \\
\hline 35. Taiwan & 3.45 & 6.89 & 6.62 & 7.01 & 6.04 & 11.35 & 8.53 & 4.69 \\
\hline 36. Korea & 3.39 & 3.55 & 4.30 & 6.41 & 9.74 & 8.19 & 5.31 & 5.84 \\
\hline 37. Greece & 4.14 & 7.92 & 5.99 & 6.41 & 5.67 & 8.94 & 8.08 & 6.48 \\
\hline 38. Panama & 2.59 & 3.23 & 1.92 & 5.06 & 2.55 & 2.01 & 436 & 2.84 \\
\hline 39. Thailand & 2.62 & 4.08 & 3.23 & 4.98 & 6.84 & 5.07 & 5.49 & 3.92 \\
\hline 40. Sí Lanka & -.03 & 8.38 & 3.29 & 3.62 & 6.92 & 8.43 & 4.42 & 1.24 \\
\hline 41. Thailand* & 2.15 & 5.30 & 1.06 & 10.16 & 3.70 & 6.55 & 5.59 & 7.16 \\
\hline 42. Korea* & 1.94 & 2.35 & 2.27 & 6.88 & 4.95 & 11.55 & 5.69 & 3.31 \\
\hline Mean & 1.83 & 3.14 & 3.34 & 4.36 & 4.42 & 5.34 & 4.31 & 3.83 \\
\hline
\end{tabular}

See note to Table 1.

Source: Chen (1993). 
TABLE 4

DIVISIA PRICE AND VOLUME INDEXES

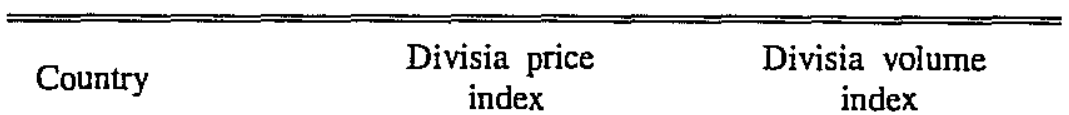

(1)

(2)

(3)

\begin{tabular}{|c|c|c|}
\hline 1. USA & 6.46 & 2.09 \\
\hline 2. Switzerland & 4.57 & 2.11 \\
\hline 3. Canada & 5.25 & 2.96 \\
\hline 4. USA* & 1.89 & 2.47 \\
\hline 5. Australia & 8.42 & 2.30 \\
\hline 6. Sweden & 8.30 & 1.58 \\
\hline 7. Denmark & 8.93 & 1.44 \\
\hline 8. France & 7.50 & 3.58 \\
\hline 9. Norway & 7.06 & 2.67 \\
\hline 10. UK & 11.27 & 1.77 \\
\hline 11. Germany & 3.96 & 3.18 \\
\hline 12. Japan & 7.81 & 3.15 \\
\hline 13. Israel & 53.54 & 2.70 \\
\hline 14. Italy & 12.39 & 2.86 \\
\hline 15. Belgium & 5.12 & 3.09 \\
\hline 16. Austria & 5.25 & 3.31 \\
\hline 17. Australia* & 2.51 & 1.75 \\
\hline 18. Hong Kong & 8.68 & 5.31 \\
\hline 19. Sweden* & 3.52 & 2.83 \\
\hline 20. Netherlands & 4.06 & 4.29 \\
\hline 21. Finland & 6.98 & 3.94 \\
\hline 22. Iceland & 11.93 & 4.90 \\
\hline 23. UK* & 2.88 & 2.52 \\
\hline 24. Singapore & 3.71 & 5.68 \\
\hline 25. Spain & 9.99 & 4.50 \\
\hline 26. Mexico & 22.02 & 1.78 \\
\hline 27. Israel & 5.85 & 6.30 \\
\hline 28. Italy* & 2.82 & 4.94 \\
\hline 29. Puerto Rico & 5.74 & 2.22 \\
\hline 30. Malta & 5.31 & 2.97 \\
\hline 31. Puerto Rico* & 2.40 & 2.49 \\
\hline 32. Ireland & 3.11 & 2.97 \\
\hline 33. South Africa & 2.23 & 1.67 \\
\hline 34. Colombia & 21.07 & 1.94 \\
\hline 35. Taiwan & 6.02 & 5.32 \\
\hline 36. Korea & 15.83 & 4.57 \\
\hline 37. Greece & 1.93 & 5.71 \\
\hline 38. Panama & 1.21 & 2.76 \\
\hline 39. Thailand & 7.63 & 3.61 \\
\hline 40. Sri Lanka & 8.22 & 2.06 \\
\hline 41. Thailand* & 1.91 & 3.36 \\
\hline 42. Korea* & 13.36 & 2.93 \\
\hline Mean & 8.06 & 3.20 \\
\hline
\end{tabular}

See note to Table 1.

Source: Chen (1993). 
vector of 42 unit elements. Equation (4.2) for $c=1, \ldots, 42$ can then be expressed in vector form as

$$
\mathbf{y}_{\mathrm{i}}=\mathbf{X}_{\mathrm{i}} \theta_{\mathrm{i}}+\varepsilon_{\mathrm{i}},
$$

where $\theta_{i}=\left[\beta_{i} \mu_{i} \lambda \alpha_{i}\right]^{\prime}$ is a vector of parameters; and $\varepsilon_{i}=\left[\varepsilon_{i, 1}, \ldots, \varepsilon_{i, 42}\right]^{\prime}$ is a vector of disturbances for commodity $i$. As one equation is redundant, we consider (4.3) for $i=1, \ldots, 7$ and write it as

$$
y=X \theta+\varepsilon,
$$

where $\mathbf{y}=\left[\mathbf{y}_{1}^{\prime}, \ldots, \mathbf{y}_{7}^{\prime}\right]^{\prime} ; \mathbf{X}=\operatorname{diag}\left[\mathbf{X}_{1}, \ldots, \mathbf{X}_{7}\right] ; \theta=\left[\theta_{1}^{\prime}, \ldots, \theta_{7}^{\prime}\right]^{\prime}$ and $\varepsilon=\left[\varepsilon_{1}^{\prime}, \ldots, \varepsilon_{7}^{\prime}\right]^{\prime}$.

Let $\varepsilon^{\mathrm{c}}=\left[\varepsilon_{1 \mathrm{c}}, \ldots, \varepsilon_{7 \mathrm{c}}\right]^{\prime}$ be a vector of the 7 disturbances for country c. If $\varepsilon^{1}, \ldots, \varepsilon^{42}$ are independent normal vectors with zero mean and non-singular covariance matrix $\Sigma$, the log-likelihood function for model (4.4) is then

$$
\ell(\theta, \Sigma ; y)=-\frac{42 \times 7}{2} \ln (2 \pi)-\frac{42}{2} \ln |\Sigma|-\frac{1}{2}(y-X \theta)^{\prime}\left(\Sigma^{-1} \otimes I\right)(y-X \theta),
$$

with

$$
\frac{\partial \ell}{\partial \theta^{\prime}}=(\mathbf{y}-\mathbf{X} \theta)^{\prime}\left(\Sigma^{-1} \otimes \mathbf{I}\right) \frac{\partial(\mathbf{X} \theta)}{\partial \theta^{\prime}}
$$

The $(i, j)^{\text {th }}$ block of the matrix $\partial(\mathbf{X} \theta) / \partial \theta^{\prime}$ is

$$
\frac{\partial\left(\mathbf{X}_{\mathrm{i}} \theta_{\mathrm{i}}\right)}{\partial \theta_{\mathrm{j}}^{\prime}}=\left[\delta_{\mathrm{ij}} \mathbf{D Q}, \lambda \frac{\partial \mathbf{z}_{\mathrm{i}}}{\partial \mu_{\mathrm{j}}}, \mathbf{z}_{\mathrm{i}}, \delta_{\mathrm{ij}} \mathrm{l}\right] \text {, }
$$


with $\partial \mathbf{z}_{\mathrm{i}} / \partial \mu_{\mathrm{j}}=\left[\partial \mathrm{z}_{\mathrm{ic}} / \partial \mu_{\mathrm{j}}\right]$ and

$$
\frac{\partial \mathrm{z}_{\mathrm{ic}}}{\partial \mu_{\mathrm{j}}}=-\left(\mu_{\mathrm{i}}+\overline{\mathrm{w}}_{\mathrm{ic}}\right) \mathrm{D} \mathrm{p}_{\mathrm{jc}}^{*}+\delta_{\mathrm{ij}}\left[\mathrm{D}_{\mathrm{ic}}^{*}-\sum_{\mathrm{k}=1}^{7}\left(\mu_{\mathrm{k}}+\overline{\mathrm{w}}_{\mathrm{kc}}\right) \mathrm{D} \mathrm{p}_{\mathrm{kc}}^{*}\right]
$$

where $\delta_{\mathrm{ij}}$ is the Kronecker delta. By setting $\partial \ell / \partial \theta^{\prime}=\mathbf{0}$, we obtain the ML estimate of the vector of parameters, $\hat{\theta}$, by using Newton's method.

As the information matrix of the likelihood function is block-diagonal with $\mathbf{E}(\mathbf{y}-\mathbf{X} \theta)=\mathbf{E}(\varepsilon)=\mathbf{0}$, the asymptotic covariance matrix of $\hat{\theta}$ is

$$
\left[-E\left[\frac{\partial^{2} \ell}{\partial \theta \partial \theta^{\prime}}\right]_{\substack{\theta=\bar{\theta} \\ \Sigma=\bar{\Sigma}}}\right]^{-1}=\left[\frac{\partial(\mathbf{X} \theta)^{\prime}}{\partial \theta}\left(\Sigma^{-1} \otimes \mathbf{I}\right) \frac{\partial(\mathbf{X} \theta)}{\partial \theta^{\prime}}\right]_{\substack{\theta=\hat{\theta} \\ \Sigma=\bar{\Sigma}}}^{-1},
$$

where $\hat{\Sigma}$ is the ML estimate of $\Sigma$, that is $\hat{\Sigma}=(1 / 42)\left[\mathbf{e}_{1}, \ldots, \mathbf{e}_{7}\right]^{\prime}\left[\mathbf{e}_{1}, \ldots, \mathbf{e}_{7}\right]$, with $\mathbf{e}_{\mathbf{i}}=\mathbf{y}_{\mathbf{i}}-\mathbf{X}_{\mathbf{i}} \hat{\theta}_{i}$, the vector of 42 residuals for commodity $i$.

As models (2.2) and (2.3) are special cases of model (2.1), they can be estimated in a similar way by imposing appropriate restrictions on the parameter vector $\theta$. The estimation procedure for model (2.4) involves a simple modification by redefining the terms $y_{i c}=D q_{i c}, \quad z_{i c}=D p_{i c}-\sum_{j=1}^{8} \bar{w}_{j c} D p_{j c}, \quad \theta_{i}=\left[\begin{array}{ll}\eta_{i} & \psi_{i} \\ \alpha_{j}\end{array}\right]^{\prime}$ and $\mathbf{X}_{i}=\left[\mathbf{D Q}, \mathbf{Z}_{\mathrm{i}}, \mathbf{l}\right]$. The vector form of equation (2.4) for $\mathrm{c}=1, \ldots, 42$ and $\mathrm{i}=1, \ldots, 8$ can then be written as equation (4.4). ${ }^{7}$ Since model (2.4) is linear, the term $\partial(\mathbf{X} \theta) / \partial \theta^{\prime}$ in the derivative of the likelihood function becomes simply $\mathbf{X}$.

\footnotetext{
${ }^{7}$ Unlike the other models, equation (2.4) for $i=1, \ldots, 8$ does not satisfy the budget constraint. Accordingly, as the disturbance covariance matrix is not singular, we retain all 8 equations in estimation.
} 


\section{THE DATA-BASED ESTIMATES}

Table 5 contains the $\mathrm{ML}$ estimates of each of the four demand systems. As can be seen, the estimates of models (2.1)-(2.3) display some similarities. The intercepts are not significant for three of the eight commodities -- Medicine, Recreation and Other. The exception to this rule is Medicine in the Working's model under preference independence. Also, the income coefficients are not significant for four of the eight commodities -- Clothing, Medicine, Transport and Other. This implies that the corresponding income elasticities are not significantly different from one. The estimates of $\mu_{i}$ in the Working's model under substitution independence are significantly different from zero for two of the eight commodities -- Housing and Durables. The estimates of $\lambda, \phi$ and $\gamma$ are not too dissimilar and it is to be noted that the $\phi$-estimate is insignificantly different from $-1 / 2$, the central-tendency value of prior estimates of the income flexibility. ${ }^{8}$ Further, most of the elasticity estimates in the constant elasticity model are significant.

The income and own-price elasticities implied by the estimates are given in Tables 6 and 7. As can be seen, Food and Housing are necessities in the 42 countries under all of the four models, while the other six commodities are luxuries. In general, the elasticities are similar across the four models. The only exception is the income elasticity estimate for Housing implied by Working's model under preference independence, which displays some erratic behaviour for some of the low-income countries. There are also some problems with the own-price elasticities for Housing for Working's model under substitution independence and Working's model under preference independence, where this elasticity is positive in some countries.

\footnotetext{
${ }^{8}$ For a survey, see Clements and S. Selvanathan (1994).
} 
TABLE 5

ESTIMATES OF FOUR DEMAND SYSTEMS

(Asymptotic standard errors in parentheses)

\begin{tabular}{|c|c|c|c|c|c|c|c|c|}
\hline $\begin{array}{l}\text { Coefficient } \\
\text { (1) }\end{array}$ & $\begin{array}{l}\text { Food } \\
\text { (2) }\end{array}$ & $\begin{array}{l}\text { Clothing } \\
\text { (3) }\end{array}$ & $\begin{array}{l}\text { Housing } \\
\text { (4) }\end{array}$ & $\begin{array}{c}\text { Durables } \\
\text { (5) }\end{array}$ & $\begin{array}{c}\text { Medicine } \\
(6)\end{array}$ & $\begin{array}{c}\text { Transport } \\
\text { (7) }\end{array}$ & $\begin{array}{l}\text { Recreation } \\
\text { (8) }\end{array}$ & $\begin{array}{l}\text { Other } \\
\text { (9) }\end{array}$ \\
\hline \multicolumn{9}{|c|}{ Working's Model under Substitution Independence } \\
\hline $\begin{array}{l}\text { Intercept } \\
\alpha_{i} \times 100\end{array}$ & $\begin{array}{l}-.190 \\
(.090)\end{array}$ & $\begin{array}{l}-.126 \\
(.054)\end{array}$ & $\begin{array}{l}.157 \\
(.062)\end{array}$ & $\begin{array}{l}-.144 \\
(.044)\end{array}$ & $\begin{array}{l}.066 \\
(.039)\end{array}$ & $\begin{array}{l}.151 \\
(.063)\end{array}$ & $\begin{array}{l}-.006 \\
(.039)\end{array}$ & $\begin{array}{c}.082 \\
(.065)\end{array}$ \\
\hline $\begin{array}{c}\text { Income } \\
\text { coefficient } \\
\beta_{1} \times 10\end{array}$ & $\begin{array}{l}-.879 \\
(.261)\end{array}$ & $\begin{array}{c}.209 \\
(.146)\end{array}$ & $\begin{array}{l}-.379 \\
(.178)\end{array}$ & $\begin{array}{l}.441 \\
(.124)\end{array}$ & $\begin{array}{c}.045 \\
(.112)\end{array}$ & $\begin{array}{c}.212 \\
(.181)\end{array}$ & $\begin{array}{c}.263 \\
(.113)\end{array}$ & $\begin{array}{c}.087 \\
(.172)\end{array}$ \\
\hline$\mu_{\mathrm{i}}$ & $\begin{array}{l}-.065 \\
(.097)\end{array}$ & $\begin{array}{c}.031 \\
(.031)\end{array}$ & $\begin{array}{l}-.179 \\
(.027)\end{array}$ & $\begin{array}{c}.110 \\
(.042)\end{array}$ & $\begin{array}{l}-.001 \\
(.020)\end{array}$ & $\begin{array}{c}.004 \\
(.045) \\
\end{array}$ & $\begin{array}{c}.018 \\
(.028) \\
\end{array}$ & $\begin{array}{c}.081 \\
(.064)\end{array}$ \\
\hline \multicolumn{9}{|c|}{$\lambda=-.433(.066)$} \\
\hline \multicolumn{9}{|c|}{ Working's Model under Preference Independence } \\
\hline $\begin{array}{l}\text { Intercept } \\
\alpha_{i} \times 100\end{array}$ & $\begin{array}{l}-.216 \\
(.085)\end{array}$ & $\begin{array}{l}-.126 \\
(.053)\end{array}$ & $\begin{array}{c}.266 \\
(.058)\end{array}$ & $\begin{array}{l}-.168 \\
(.042)\end{array}$ & $\begin{array}{c}.067 \\
(.033)\end{array}$ & $\begin{array}{l}.130 \\
(.056)\end{array}$ & $\begin{array}{c}.016 \\
(.037)\end{array}$ & $\begin{array}{c}.032 \\
(.051)\end{array}$ \\
\hline $\begin{array}{c}\text { Income } \\
\text { coefficient } \\
\beta_{i} \times 10\end{array}$ & $\begin{array}{l}-.807 \\
(.245)\end{array}$ & $\begin{array}{c}.218 \\
(.139)\end{array}$ & $\begin{array}{r}-.683 \\
(.167)\end{array}$ & $\begin{array}{l}.578 \\
(.113)\end{array}$ & $\begin{array}{c}.042 \\
(.098)\end{array}$ & $\begin{array}{c}.275 \\
(.165)\end{array}$ & $\begin{array}{c}.227 \\
(.107)\end{array}$ & $\begin{array}{l}.152 \\
(.159)\end{array}$ \\
\hline \multicolumn{9}{|c|}{$\phi=-.424(.053)$} \\
\hline \multicolumn{9}{|c|}{ Selvanathan's Model } \\
\hline $\begin{array}{l}\text { Intercept } \\
\alpha_{i} \times 100\end{array}$ & $\begin{array}{l}-.197 \\
(.085)\end{array}$ & $\begin{array}{l}-.097 \\
(.051)\end{array}$ & $\begin{array}{l}.175 \\
(.073)\end{array}$ & $\begin{array}{l}-.119 \\
(.047)\end{array}$ & $\begin{array}{c}.064 \\
(.038)\end{array}$ & $\begin{array}{c}.136 \\
(.062)\end{array}$ & $\begin{array}{c}.007 \\
(.040)\end{array}$ & $\begin{array}{c}.031 \\
(.060)\end{array}$ \\
\hline $\begin{array}{c}\text { Income } \\
\text { coefficient } \\
\beta_{i} \times 10\end{array}$ & $\begin{array}{r}-.896 \\
(.246)\end{array}$ & $\begin{array}{c}.159 \\
(.147)\end{array}$ & $\begin{array}{l}-.401 \\
(.210)\end{array}$ & $\begin{array}{c}.502 \\
(.135)\end{array}$ & $\begin{array}{c}.040 \\
(.111)\end{array}$ & $\begin{array}{c}.231 \\
(.181)\end{array}$ & $\begin{array}{l}.246 \\
(.115)\end{array}$ & $\begin{array}{c}.119 \\
(.174)\end{array}$ \\
\hline \multicolumn{9}{|c|}{$\gamma=-.386(.058)$} \\
\hline \multicolumn{9}{|c|}{ Constant Elasticity Model } \\
\hline $\begin{array}{l}\text { Intercept } \\
\alpha_{i} \times 100\end{array}$ & $\begin{array}{l}-.656 \\
(.230)\end{array}$ & $\begin{array}{l}-1.359 \\
(.634)\end{array}$ & $\begin{array}{c}.778 \\
(.453)\end{array}$ & $\begin{array}{l}-1.661 \\
(.607)\end{array}$ & $\begin{array}{l}1.303 \\
(.706)\end{array}$ & $\begin{array}{l}.664 \\
(.764)\end{array}$ & $\begin{array}{l}-.067 \\
(.482)\end{array}$ & $\begin{array}{l}.538 \\
(.698)\end{array}$ \\
\hline $\begin{array}{c}\text { Income } \\
\text { elasticity } \\
\eta_{i}\end{array}$ & $\begin{array}{l}.773 \\
(.067)\end{array}$ & $\begin{array}{l}1.194 \\
(.174)\end{array}$ & $\begin{array}{c}.814 \\
(.132)\end{array}$ & $\begin{array}{l}1.569 \\
(.175)\end{array}$ & $\begin{array}{l}1.080 \\
(.205)\end{array}$ & $\begin{array}{l}1.484 \\
(.221)\end{array}$ & $\begin{array}{l}1.322 \\
(.140)\end{array}$ & $\begin{array}{l}1.138 \\
(.192)\end{array}$ \\
\hline$\psi_{i}$ & $\begin{array}{l}-.319 \\
(.103)\end{array}$ & $\begin{array}{l}-.559 \\
(.119)\end{array}$ & $\begin{array}{l}-.259 \\
(.067)\end{array}$ & $\begin{array}{l}-.920 \\
(.150)\end{array}$ & $\begin{array}{l}-.655 \\
(.123)\end{array}$ & $\begin{array}{l}-.441 \\
(.223)\end{array}$ & $\begin{array}{l}-.487 \\
(.111)\end{array}$ & $\begin{array}{l}-.453 \\
(.226)\end{array}$ \\
\hline
\end{tabular}


TABLE 6

INCOME ELASTICITIES

\begin{tabular}{|c|c|c|c|c|c|c|c|c|c|c|c|c|c|c|c|c|}
\hline \multirow{2}{*}{ Country } & \multicolumn{4}{|c|}{ Food } & \multicolumn{4}{|c|}{ Clothing } & \multicolumn{4}{|c|}{ Housing } & \multicolumn{4}{|c|}{ Durables } \\
\hline & SI & PI & SM & CEM & SI & PI & SM & CEM & SI & PI & $S M$ & CEM & SI & PI & SM & CEM \\
\hline 1. USA & .50 & 54 & .49 & .77 & 1.27 & 1.29 & 1.21 & 1.19 & .80 & .65 & .79 & .81 & 1.64 & 1.84 & 1.73 & 1.57 \\
\hline 2. Switzerland & .73 & .75 & .72 & .77 & 1.31 & 1.32 & 1.24 & 1.19 & .79 & .62 & .78 & .81 & 1.57 & 1.74 & 1.64 & 1.57 \\
\hline 3. Canada & .61 & .64 & .60 & .77 & 1.25 & 1.26 & 1.19 & 1.19 & .80 & .64 & .79 & .81 & 1.51 & 1.66 & 1.57 & 1.57 \\
\hline 4. USA & .67 & .70 & .66 & .77 & 1.22 & 1.23 & 1.17 & 1.19 & .83 & .70 & .82 & .81 & 1.60 & 1.79 & 1.69 & 1.57 \\
\hline 5. Australia & .67 & .70 & .67 & .77 & 1.24 & 1.26 & 1.19 & 1.19 & .77 & 59 & .76 & .81 & 1.53 & 1.70 & 1.60 & 1.57 \\
\hline 6. Sweden & .68 & .71 & .68 & .77 & 1.26 & 1.27 & 1.20 & 1.19 & .84 & .71 & .83 & .81 & 1.57 & 1.75 & 1.65 & 1.57 \\
\hline 7. Denmark & .69 & .71 & .68 & .77 & 1.30 & 1.31 & 1.23 & 1.19 & .82 & .67 & .81 & .81 & 1.49 & 1.64 & 1.56 & 1.57 \\
\hline 8. France & .66 & .69 & .65 & .77 & 1.25 & 1.27 & 1.19 & 1.19 & .74 & .53 & .72 & .81 & 1.43 & 1.56 & 1.49 & 1.57 \\
\hline 9. Norway & .72 & .74 & .71 & .77 & 1.21 & 1.22 & 1.16 & 1.19 & .74 & .53 & .72 & .81 & 150 & 1.65 & 1.57 & 1.57 \\
\hline 10. UK & .64 & .67 & .63 & .77 & 1.26 & 1.27 & 1.20 & 1.19 & .80 & .64 & .79 & .81 & 1.58 & 1.76 & 1.66 & 1.57 \\
\hline 11. Germany & .71 & .73 & .70 & .77 & 1.20 & 1.21 & 1.15 & 1.19 & .76 & .57 & .75 & .81 & 1.37 & 1.48 & 1.42 & 1.57 \\
\hline 12. Japan & .68 & .71 & .67 & .77 & 1.27 & 1.28 & 1.21 & 1.19 & .77 & .59 & .76 & .81 & 1.65 & 1.86 & 1.74 & 1.57 \\
\hline 13. Israel & .70 & .72 & .69 & .77 & 1.30 & 1.32 & 1.23 & 1.19 & .83 & .69 & .82 & .81 & 1.35 & 1.46 & 1.40 & 157 \\
\hline 14. Italy & .75 & .77 & .75 & .77 & 1.22 & 123 & 1.17 & 1.19 & .71 & .48 & 69 & .81 & 1.63 & 1.83 & 1.72 & 1.57 \\
\hline 15. Belgium & .71 & .73 & .70 & .77 & 1.28 & 129 & 1.21 & 1.19 & .76 & .57 & .74 & .81 & 1.31 & 1.41 & 1.36 & 1.57 \\
\hline 16. Austria & .70 & .72 & .69 & .77 & 1.18 & 1.19 & 1.14 & 1.19 & .68 & .43 & .67 & .81 & 1.50 & 1.65 & 1.56 & 1.57 \\
\hline 17. Australia* & .74 & .76 & .73 & .77 & 1.19 & 1.20 & 1.14 & 1.19 & .70 & .46 & .68 & .81 & 1.57 & 1.74 & 1.64 & 1.57 \\
\hline 18. Hong Kong & .70 & .72 & .69 & .77 & 1.11 & 1.12 & 1.08 & 1.19 & .74 & .53 & .72 & .81 & 1.44 & 1.57 & 1.50 & 1.57 \\
\hline 19. Sweden* & .76 & .78 & .76 & .77 & 1.18 & 1.19 & 1.14 & 1.19 & .76 & .57 & .75 & .81 & 1.63 & 1.83 & 1.72 & 1.57 \\
\hline 20. Netherlands & .73 & .75 & .73 & .77 & 1.14 & 1.15 & 1.11 & 1.19 & .65 & .37 & .63 & .81 & 1.35 & 1.46 & 1.40 & 1.57 \\
\hline 21. Finland & .76 & .78 & .76 & .77 & 125 & 126 & 1.19 & 1.19 & .74 & .52 & .72 & .81 & 159 & 1.77 & 1.67 & 1.57 \\
\hline 22. Iceland & .72 & .74 & .71 & .77 & 1.20 & 1.20 & 1.15 & 1.19 & .80 & .64 & .79 & .81 & 1.44 & 158 & 1.50 & 1.57 \\
\hline 23. UK* & .78 & .80 & .77 & .77 & 1.19 & 120 & 1.15 & 1.19 & .79 & .63 & .78 & .81 & 1.67 & 1.88 & 1.76 & 1.57 \\
\hline 24. Singapore & .69 & .72 & .69 & .77 & 1.23 & 1.25 & 1.18 & 1.19 & .61 & .29 & .58 & .81 & 1.52 & 1.68 & 1.59 & 1.57 \\
\hline 25. Sраіл & .77 & .79 & .76 & .77 & 1.20 & 1.21 & 1.15 & 1.19 & .73 & .51 & .71 & .81 & 1.52 & 1.68 & 1.59 & 1.57 \\
\hline 26. Mexico & .77 & .79 & .77 & .77 & 1.19 & 1.20 & 1.14 & 1.19 & .62 & .31 & .60 & .81 & 1.36 & 1.47 & 1.41 & 1.57 \\
\hline 27. Israel & .72 & .75 & .72 & .77 & 1.22 & 1.23 & 1.17 & 1.19 & .80 & .64 & .79 & .81 & 1.60 & 1.78 & 1.68 & 1.57 \\
\hline 28. Italy* & .81 & .83 & .81 & .77 & 1.21 & 122 & 1.16 & 1.19 & .77 & .59 & .76 & .81 & 2.38 & 2.81 & 2.57 & 1.57 \\
\hline 29. Puerto Rico & .72 & .74 & .71 & .77 & 1.21 & 1.22 & 1.16 & 1.19 & .73 & 51 & .71 & .81 & 1.52 & 1.69 & 1.60 & 1.57 \\
\hline 30. Malta & .76 & .78 & .75 & .77 & 1.24 & 1.25 & 1.18 & 1.19 & .44 & -.01 & .41 & .81 & 1.40 & 1.53 & 1.46 & 1.57 \\
\hline 31. Puerto Rico* & .75 & .77 & .75 & .77 & 1.20 & 1.21 & 1.15 & 1.19 & .75 & .55 & .74 & .81 & 1.64 & 1.84 & 1.73 & 1.57 \\
\hline 32. Ireland & .82 & .84 & .82 & .77 & 1.21 & 122 & 1.16 & 1.19 & .70 & .47 & .69 & .81 & 1.77 & 2.01 & 1.88 & 1.57 \\
\hline 33. South Africa & .76 & .78 & .76 & .77 & 1.18 & 1.18 & 1.14 & 1.19 & .77 & .59 & .76 & .81 & 1.57 & 1.74 & 1.64 & 1.57 \\
\hline 34. Colombia & .78 & .80 & .77 & .77 & 1.28 & 1.29 & 1.21 & 1.19 & .69 & .45 & .68 & .81 & 1.78 & 2.01 & 1.88 & 1.57 \\
\hline 35. Taiwan & .82 & .83 & .82 & .77 & 1.39 & 1.41 & 1.30 & 1.19 & .77 & 58 & .75 & .81 & 1.93 & 2.22 & 2.06 & 1.57 \\
\hline 36. Korea & .83 & .84 & .82 & .77 & 1.24 & 1.25 & 1.19 & 1.19 & .57 & .23 & .55 & .81 & 1.95 & 2.24 & 2.08 & 1.57 \\
\hline 37. Greece & .81 & .83 & .81 & .77 & 1.17 & 1.18 & 1.13 & 1.19 & .79 & .63 & .78 & .81 & 2.13 & 2.48 & 2.29 & 1.57 \\
\hline 38. Panzma & .81 & .82 & .80 & .77 & 1.28 & 1.29 & 1.22 & 1.19 & .77 & 59 & .76 & .81 & 1.69 & 1.90 & 1.78 & 1.57 \\
\hline 39. Thailand & .83 & .85 & .83 & .77 & 122 & 1.23 & 1.17 & 1.19 & .44 & -.01 & .41 & .81 & 1.77 & 2.01 & 1.88 & 1.57 \\
\hline 40. Sri Lanka & .86 & .87 & .86 & .77 & 1.32 & 1.33 & 1.24 & 1.19 & .41 & -.07 & .37 & $.8 \mathrm{I}$ & 2.00 & 2.30 & 2.13 & 1.57 \\
\hline 41. Thailand* & .85 & .86 & .84 & .77 & 1.25 & 1.26 & 1.19 & 1.19 & .53 & .15 & 50 & .81 & 2.38 & 2.81 & 2.57 & 1.57 \\
\hline 42. Korea* & .85 & .87 & .85 & .77 & 1.20 & 1.21 & 1.15 & 1.19 & .67 & .40 & .65 & .81 & 2.58 & 3.06 & 2.79 & 1.57 \\
\hline Mean & .74 & .76 & .73 & .77 & 1.23 & 1.24 & 1.18 & 1.19 & .72 & .49 & .70 & .81 & 1.65 & 1.85 & 1.74 & 1.57 \\
\hline
\end{tabular}


TABLE 6 (continued)

\section{INCOME ELASTICITIES}

\begin{tabular}{|c|c|c|c|c|c|c|c|c|c|c|c|c|c|c|c|c|}
\hline \multirow{2}{*}{ Country } & \multicolumn{4}{|c|}{ Medicine } & \multicolumn{4}{|c|}{ Transport } & \multicolumn{4}{|c|}{ Recreation } & \multicolumn{4}{|c|}{ Other } \\
\hline & SI & PI & SM & CEM & SI & PI & SM & CEM & SI & PI & SM & CEM & SI & PI & SM & CEM \\
\hline 1. USA & 1.04 & 1.04 & 1.04 & 1.08 & 1.13 & 1.17 & 1.14 & 1.48 & 1.31 & 1.27 & 1.29 & 1.32 & 1.07 & 1.12 & 1.09 & 1.14 \\
\hline 2. Switzerland & 1.07 & 1.06 & 1.06 & 1.08 & 1.19 & 1.25 & 1.21 & 1.48 & 1.28 & 1.24 & 1.26 & 1.32 & 1.10 & 1.18 & 1.14 & 1.14 \\
\hline 3. Canadin & 1.12 & 1.11 & 1.10 & 1.08 & 1.14 & 1.18 & 1.15 & 1.48 & 1.32 & 1.27 & 1.30 & 1.32 & 1.06 & 1.11 & 1.08 & 1.14 \\
\hline 4. USA* & 1.06 & 1.05 & 1.05 & 1.08 & 1.14 & 1.18 & 1.15 & 1.48 & 1.48 & 1.41 & 1.45 & 1.32 & 1.18 & 1.31 & 1.24 & 1.14 \\
\hline 5. Australia & 1.07 & 1.07 & 1.06 & 1.08 & 1.14 & 1.18 & 1.15 & 1.48 & 1.38 & 1.33 & 1.36 & 1.32 & 1.08 & 1.14 & 1.11 & 1.14 \\
\hline 6. Sweden & 1.20 & 1.19 & 1.18 & 1.08 & 1.15 & 1.20 & 1.17 & 1.48 & 1.27 & 1.23 & 1.25 & 1.32 & 1.12 & 1.22 & 1.17 & 1.14 \\
\hline 7. Denmark & 1.24 & 1.22 & 1.21 & 1.08 & 1.14 & 1.18 & 1.15 & 1.48 & 1.30 & 1.26 & 1.28 & 1.32 & 1.09 & 1.17 & 1.13 & 1.14 \\
\hline 8. France & 1.04 & 1.04 & 1.04 & 1.08 & 1.18 & 1.23 & 1.19 & 1.48 & 1.42 & 1.36 & 1.39 & 1.32 & 1.07 & 1.12 & 1.10 & 1.14 \\
\hline 9. Norway & 1.11 & 1.10 & 1.09 & 1.08 & 1.16 & 1.21 & 1.17 & 1.48 & 1.32 & 1.28 & 1.30 & 1.32 & 1.09 & 1.15 & 1.12 & 1.14 \\
\hline 10. UK & 1.55 & 1.51 & 1.48 & 1.08 & 1.16 & 1.20 & 1.17 & 1.48 & 1.26 & 1.22 & 1.24 & 1.32 & 1.05 & 1.09 & 1.07 & 1.14 \\
\hline 11. Germany & 1.17 & 1.16 & 1.15 & 1.08 & 1.16 & 1.21 & 1.18 & 1.48 & 1.35 & 1.30 & 1.33 & 1.32 & 1.10 & 1.18 & 1.14 & 1.14 \\
\hline 12. Japan & 1.05 & 1.05 & 1.04 & 1.08 & 1.24 & 1.31 & 1.26 & 1.48 & 1.29 & 1.25 & 1.27 & 1.32 & 1.06 & 1.11 & 1.08 & 1.14 \\
\hline 13. Istael & 1.13 & 1.12 & 1.11 & 1.08 & 1.19 & 1.25 & 1.21 & 1.48 & 1.47 & 1.40 & 1.44 & 1.32 & 1.10 & 1.17 & 1.13 & 1.14 \\
\hline 14. Italy & 1.12 & 1.11 & 1.10 & 1.08 & 1.19 & 1.25 & 1.21 & 1.48 & 1.36 & 1.31 & 1.33 & 1.32 & 1.07 & 1.12 & 1.09 & 1.14 \\
\hline 15. Belgium & 1.07 & 1.06 & 1.06 & 1.08 & 1.20 & 1.26 & 1.22 & 1.48 & 1.63 & 1.54 & 1.58 & 1.32 & 1.08 & 1.14 & 1.11 & 1.14 \\
\hline 16. Austrin & 1.13 & 1.12 & 1.11 & 1.08 & 1.16 & 1.21 & 1.17 & 1.48 & 1.44 & 1.38 & 1.41 & 1.32 & 1.06 & 1.10 & 1.08 & 1.14 \\
\hline 17. Australia* & 1.08 & 1.07 & 1.07 & 1.08 & 1.16 & 1.21 & 1.18 & 1.48 & 1.61 & 1.53 & 1.57 & 1.32 & 1.07 & 1.13 & 1.10 & 1.14 \\
\hline 18. Hong Kong & 1.08 & 1.08 & 1.07 & 1.08 & 1.28 & 1.36 & $1.3 i$ & & & 1.28 & 1.30 & & 1.13 & 1.23 & 1.18 & 1.14 \\
\hline 19. Sweden* & 1.12 & 1.11 & 1.11 & 1.08 & 1.15 & 1.19 & 1.16 & 1.48 & 1.30 & 1.25 & 1.28 & 1.32 & 1.36 & 1.63 & 1.50 & 1.14 \\
\hline 20. Netherlands & 1.07 & 1.06 & 1.06 & 1.08 & 1.32 & 1.41 & 1.35 & 1.48 & 1.40 & 1.35 & 1.38 & 1.32 & 1.09 & 1.16 & 1.13 & 1.14 \\
\hline 21. Finland & 1.18 & 1.16 & 1.16 & 1.08 & 1.16 & 1.20 & 1.17 & 1.48 & 1.36 & 1.31 & 1.33 & 1.32 & 1.09 & 1.16 & 1.13 & 1.14 \\
\hline 22. Iceland & 1.07 & 1.07 & 1.07 & 1.08 & 1.18 & 1.23 & 1.19 & 1.48 & 1.46 & 1.40 & 1.43 & 1.32 & 1.17 & 1.30 & 1.24 & 1.14 \\
\hline 23. UK* & 1.20 & 1.18 & 1.17 & 1.08 & 1.19 & 1.25 & 1.21 & 1.48 & 1.35 & 1.30 & 1.33 & 1.32 & 122 & 1.38 & 1.30 & 1.14 \\
\hline 24. Singapore & 1.17 & 1.16 & 1.15 & 1.08 & 1.17 & 122 & 1.19 & 1.48 & 1.24 & 1.21 & 1.22 & 1.32 & 1.05 & 1.08 & 1.07 & 1.14 \\
\hline 25. Spair & 1.10 & 1.09 & 1.09 & 1.08 & & 129 & 1.24 & 1.48 & 1.42 & 1.36 & 1.39 & & 1.10 & 1.17 & 1.13 & 1.14 \\
\hline 26. Mexico & 1.13 & 1.12 & 1.11 & 1.08 & 1.24 & 1.30 & 1.26 & 1.48 & 1.52 & 1.45 & 1.49 & 1.32 & 1.09 & 1.15 & 1.12 & 1.14 \\
\hline 27. Israel & 1.07 & 1.06 & 1.06 & 1.08 & 1.29 & 1.38 & 132 & 1.48 & 1.33 & 1.28 & 1.30 & 1.32 & 1.09 & 1.15 & 1.12 & 1.14 \\
\hline 28. Italy* & 1.07 & 1.07 & 1.06 & 1.08 & 1.26 & 1.34 & 1.29 & 1.48 & 1.35 & 1.30 & 1.33 & 1.32 & 1.48 & 1.84 & 1.66 & 1.14 \\
\hline 29. Puerto Rico & 1.10 & 1.10 & 1.09 & 1.08 & 1.14 & 1.19 & 1.16 & 1.48 & 1.40 & 1.34 & 1.37 & 1.32 & 1.08 & 1.14 & 1.11 & 1.14 \\
\hline 30. Malta & 1.12 & 1.11 & 1.10 & 1.08 & 1.15 & 120 & 1.17 & 1.48 & 1.40 & 1.35 & 1.37 & 1.32 & 1.07 & 1.12 & 1.09 & 1.14 \\
\hline 31. Puerto Rico* & 1.07 & 1.06 & 1.06 & 1.08 & 1.17 & 1.22 & 1.19 & 1.43 & 1.30 & 1.26 & 128 & 1.32 & 1.23 & 1.41 & 1.32 & 1.14 \\
\hline 32. Ireland & 1.35 & 1.32 & 1.31 & 1.08 & 1.24 & 1.31 & 1.26 & 1.48 & 1.42 & 1.36 & 1.39 & 1.32 & 1.15 & 1.26 & 1.20 & 1.14 \\
\hline 33. Sauth Africa & 1.09 & 1.09 & 1.08 & 1.08 & 1.16 & 1.21 & 1.18 & 1.48 & 1.56 & 1.48 & 1.52 & 1.32 & 1.21 & 1.37 & 1.29 & 1.14 \\
\hline 34. Colombia & 1.08 & 1.08 & 1.07 & 1.08 & 1.17 & 1.22 & 1.18 & 1.48 & 1.49 & 1.42 & 1.46 & 1.32 & 1.08 & 1.13 & 1.10 & 1.14 \\
\hline 35. Taiwan & 1.10 & 1.09 & 1.09 & 1.08 & 1.55 & 1.71 & 1.60 & 1.48 & 1.28 & 124 & 1.26 & 1.32 & 1.12 & 1.21 & 1.17 & 1.14 \\
\hline 36. Korea & 1.14 & 1.13 & 1.12 & 1.08 & 1.26 & 1.33 & 1.28 & 1.48 & 1.36 & 1.31 & 1.34 & 1.32 & 1.10 & 1.18 & 1.14 & 1.14 \\
\hline 37. Greece & 1.13 & 1.12 & 1.11 & 1.08 & 1.31 & 1.40 & 1.34 & 1.48 & 1.43 & 1.37 & 1.40 & 1.32 & 1.38 & 1.66 & 1.52 & 1.14 \\
\hline 38. Рanama & 1.10 & 1.09 & 1.08 & 1.08 & 1.23 & 1.29 & 1.25 & 1.48 & 1.35 & 1.30 & 1.33 & 1.32 & 1.38 & 1.66 & 1.52 & 1.14 \\
\hline 39. Thailand & 1.09 & 1.08 & 1.08 & 1.08 & 1.23 & 1.30 & 1.25 & 1.48 & 1.44 & 1.38 & 1.41 & 1.32 & 1.17 & 1.29 & 1.23 & 1.14 \\
\hline 40. Sri Lanka & 1.20 & 1.19 & 1.18 & 1.08 & 1.24 & 1.31 & 1.26 & 1.48 & 1.55 & 1.47 & 1.51 & 1.32 & 1.36 & 1.62 & 1.49 & 1.14 \\
\hline 41. Thailand* & 1.08 & 1.07 & 1.07 & 1.08 & 1.27 & 1.35 & 1.29 & 1.48 & 1.35 & 1.30 & 1.33 & 1.32 & 1.48 & 1.84 & 1.66 & 1.14 \\
\hline 42. Korea* & 1.11 & 1.10 & 1.09 & 1.08 & 1.45 & 1.58 & 1.49 & 1.48 & 1.60 & 151 & 1.56 & 1.32 & 1.39 & 1.69 & 1.54 & 1.14 \\
\hline Mean & 1.12 & 1.12 & 1.11 & 1.08 & 121 & 1.27 & 1.23 & 1.48 & 1.39 & 1.34 & 1.37 & 1.32 & 1.16 & 1.27 & 1.21 & 1.14 \\
\hline
\end{tabular}

Notes: 1 . SI $=$ Working's model under substitution independence, $\mathrm{PI}=$ Working's model under preference independence, $\mathrm{SM}=$ Selvanathan's model, and CEM = Constant elasticity model.

2. An asterisk $\left({ }^{*}\right)$ indicates a second period for a country. 
TABLE 7

OWN-PRICE ELASTICITIES

\begin{tabular}{|c|c|c|c|c|c|c|c|c|c|c|c|c|c|c|c|c|}
\hline \multirow{2}{*}{ Country } & \multicolumn{4}{|c|}{ Food } & \multicolumn{4}{|c|}{ Clothing } & \multicolumn{4}{|c|}{ Housing } & \multicolumn{4}{|c|}{ Durables } \\
\hline & SI & PI & SM & CEM & SI & PI & SM & CEM & SI & $\mathrm{PI}$ & SM & CEM & SI & PI & SM & CEM \\
\hline 1. USA & -.24 & -21 & -.32 & -.26 & -.55 &. .49 & -.36 & -.52 & -.03 & -.24 & -.31 & -21 & -.93 & -.68 & -.36 & -.86 \\
\hline 2. Switzerland & -.26 & -.24 & -.26 & -.22 & -.57 & -.51 & -.36 & -.52 & .00 & -.23 & -.32 & -.21 & -.85 & -.64 & -.36 & -.85 \\
\hline 3. Canada & -.26 & -.23 & -.30 & -.25 & -53 & -.48 & -.35 & -51 & -.02 & -24 & -31 & -.21 & -.79 & -.60 & -.35 & -.84 \\
\hline 4. USA & -.26 & -.24 & -.28 & -.23 & -.50 & -.46 & -.35 & -.51 & -.09 &. .25 & -.30 & -.20 & -.89 & -.66 & -.36 & -.85 \\
\hline 5. Australia & -.26 & -.24 & -.28 & -.23 & .52 & -.48 & -.35 & -51 & .03 & -.23 & -.32 &. .22 & -.81 & -.62 & -.35 & -.84 \\
\hline 6. Sweden & -.26 & -.24 & -.28 & -.23 & -.53 &. .48 & -.36 & -.51 & -.10 &. .25 & -30 & -.20 & -.86 & -.64 & -.36 & -.85 \\
\hline 7. Denmark & -.26 & -.24 & -.28 & -.23 & -.56 & -.51 & -.36 & -.52 & -.06 & -.25 & -.31 & -.20 & -.77 & -59 & -.35 & -.84 \\
\hline 8. France & -.26 & -.24 & -.29 & -.24 &. .53 & -.48 & -.35 & -51 & .11 & -.21 & -.33 & -.22 & -.71 & -.56 & -.35 & -.83 \\
\hline 9. Norway & -26 & -.24 & -.27 & -.22 & -.49 & -.45 & -.35 & -50 & .10 & -.21 & -.33 & -.22 & -.78 & -.60 & -.35 & -.84 \\
\hline 10. UK & -.26 & -.24 & -.29 & -24 & .53 & -.48 & -.36 & -.51 & -.02 & .24 & -31 & -.21 & -.86 & -.65 & -.36 & -.85 \\
\hline 11. Germany & -.26 & -.24 & -.27 &. .22 & -.49 & -.45 & -.35 & -.50 & .06 & -.22 & -.33 & -.22 & -.64 & -.52 & -.34 & -.81 \\
\hline 12. Japan & -.26 &. .24 & -.28 & -.23 & -.54 & -.49 & -.36 & -.52 & .03 & -.23 & -.32 & -.22 & -.94 & -.69 & -.36 & -.86 \\
\hline 13. Israel & -.26 & -.24 & -.27 & -.23 & .57 & -.51 & -.36 & -.52 & -.08 & -.25 & -.30 & -.20 & -.62 & -51 & -.34 & -.81 \\
\hline 14. Italy & -.25 & -.24 & -.25 & -.21 & -.50 & -.46 & -.35 & -.51 & .17 & -.19 & -.34 & -.23 & -.92 & -.68 & -.36 & -.86 \\
\hline 15. Belgium & -.26 & -.24 & -.27 & -.22 & .55 & -.49 & -.36 & -.52 & .06 & -.22 & -.33 & -.22 & -.58 & -.48 & -.33 & -.79 \\
\hline 16. Austria & -.26 & -.24 & -.27 & -23 & -.47 & -.44 & -.34 & -.50 & .23 & -.17 & -.34 & -.23 & -.78 & -.60 & -.35 & -.84 \\
\hline 17. Australia * & -.26 & -.24 & -.26 & -.21 & -.48 & -.44 & -.34 & -.50 & .19 & -.18 & -34 & -.23 & -.85 & -.64 & -.36 & -.85 \\
\hline 18. Hong Kong & -.26 & -.24 & -.27 & -.23 & -.39 & -.37 & -.31 & -.45 & .11 & -21 & -.33 & -.22 & -.72 & -.56 & -.35 & -.83 \\
\hline 19. Sweden* & -.25 & -.24 & -.25 & -.20 & -.47 & -.44 & -.34 & -.49 & .06 & -.22 & -.33 & -.22 & -.92 & -.68 & -.36 & -.86 \\
\hline 20. Netheriands & -.26 & -24 & -.26 & -.22 & -.43 & -.40 & -.33 & -.48 & .30 & -.15 & -.34 & -.23 & -.62 & -.51 & -.34 & -.81 \\
\hline 21. Finland & -.25 & -24 & -.24 & -.20 & .53 &. .48 & -.35 & -.51 & .11 & -.21 & -.33 & -.22 & -.87 & -.65 & -.36 & -.85 \\
\hline 22. Iceland & -.26 & -24 & -.27 & -.22 & -.48 & -.45 & -.35 & -.50 & -.03 & -.24 & -.31 & -.21 & -.72 & -.56 & -.35 & -.83 \\
\hline 23. UK* & -.24 & -.23 & -.23 & -.19 & -.48 & -.45 & -.35 & -.50 & -.01 & -.24 & -.32 & -.21 & -.95 & -.70 & -.36 & -.86 \\
\hline 24. Singapore & -.26 & -.24 & -.28 &. .23 &. .52 & -.47 & -.35 & -.51 & .41 & -.12 & -.35 & -.23 & -.80 & -.61 & -.35 & -.84 \\
\hline 25. Spain & -.25 & -.23 & -.24 & -.20 & -.48 & -.45 & -.35 & -.50 & .13 & -.20 & -.33 & -.22 & -.80 & -.61 & -.35 & -.84 \\
\hline 26. Mexico & -.24 & -.23 & -.24 & -.19 & -.48 & -.44 & -.34 &. .50 & .37 & -.13 & -35 & -.23 & -.63 & -51 & -.34 & -.81 \\
\hline 27. Israel & -.26 & -.24 & -26 &. .22 & -.51 &. .46 & -.35 & -51 & -.03 & -.24 & -.31 & -.21 & -.88 & -.66 & -.36 & -.85 \\
\hline 28. Italy* & -.22 & -.22 & -.21 & -.17 & -.49 & -.45 & -.35 & -50 & .03 & -.23 & -.32 & -.22 & -1.65 & -1.08 & -.37 & -.89 \\
\hline 29. Puerto Rico & -.26 & -.24 & -.27 & -.22 & -.50 & -.46 & -.35 & -.50 & .12 & .20 & -.33 & -.22 & -.81 & -.61 & -.35 & -.84 \\
\hline 30. Maith & -.25 & -.24 & -.25 & -.20 & -.52 & -.47 & -.35 & -.51 & .79 & .00 & -.36 & -.24 & -.68 & -.54 & -.34 & -.82 \\
\hline 31. Puerto Rico* & -.25 & -.24 & -25 & -.21 & -.48 & -.45 & -.35 & -.50 & .08 & -.22 & -.33 & -.22 & -.92 & -.68 & -.36 & -.86 \\
\hline 32. Ireland & -.22 & -.21 & -.20 & -.16 & -.49 & -.45 & -.35 & -.50 & .18 & -.19 & -.34 & -.23 & -1.06 & -.76 & -.36 & -.87 \\
\hline 33. South Africa & -.25 & -.24 & -.24 & -.20 & -.47 & -.43 & -.34 & -.49 & .03 & -.23 & -.32 & -.22 & -.85 & -.64 & -.36 & -.85 \\
\hline 34. Colombia & -.24 & -.23 & -23 & -.19 & -.55 & -.49 & -.36 & -52 & .20 & -.18 & -.34 & -23 & -1.06 & -.76 & -.36 & -.87 \\
\hline 35. Taiwan & -.22 & -.21 & -.20 & -.16 & -.63 & -.55 & -.37 & -.53 & .05 & -.22 & -.32 & -.22 & -1.21 & -.84 & -.37 & -.88 \\
\hline 36. Korea & -.21 & -.20 & -.19 & -.16 & -.52 & -.47 & -.35 & -.51 & .48 &. .10 & -35 & -.24 & -1.23 & -.85 & -.37 & -.88 \\
\hline 37. Greece & -.22 & -.22 & -.21 & -.17 & -.46 & -.43 & -.34 & -.49 & -.01 & -.24 & -32 & -.21 & -1.41 & -.95 & -.37 & -.88 \\
\hline 38. Panama & -.23 & -.22 & -.21 & -.17 & -.55 & -.50 & -.36 & -.52 & .03 & -.23 & -.32 & -.22 & -.97 & -.71 & -.36 & -.86 \\
\hline 39. Thailand & -.20 & -.20 & -.18 & -.15 & -.50 & -.46 & -.35 & -.51 & .79 & .01 & -.36 & -24 & -1.06 & -.76 & -.36 & -.87 \\
\hline 40. Sri Lanka & -.16 & -.16 & -.14 & -.11 & -.58 & -.52 & -.36 & -.52 & .87 & .03 & -.36 & -.24 & -1.28 & -.88 & -.37 & -.88 \\
\hline 41. Thailand* & -.19 & -.18 & -.16 & -.14 & -.53 & -.48 & -.35 & -.51 & .59 & -.06 & -.36 & -.24 & -1.65 & -1.08 & -.37 & -.89 \\
\hline 42. Korea* & -.18 & -.18 & -.16 & -.13 & -.49 & -.45 & -.35 & -.50 & .26 & -.16 & -34 & -23 & -1.85 & -1.19 & -.38 &. .89 \\
\hline Mean & -.24 & -.23 &.-25 & -.20 &. .51 & -.47 & -.35 & -.51 & .15 & -.19 & -.33 & -.22 & -.93 & -.68 & -.36 & -.85 \\
\hline
\end{tabular}

(Continued on next page) 
TABLE 7 (continued)

OWN-PRICE ELASTICITIES

\begin{tabular}{|c|c|c|c|c|c|c|c|c|c|c|c|c|c|c|c|c|}
\hline \multirow{2}{*}{ Country } & \multicolumn{4}{|c|}{ Medicine } & \multicolumn{4}{|c|}{ Transport } & \multicolumn{4}{|c|}{ Recreation } & \multicolumn{4}{|c|}{ Other } \\
\hline & SI & PI & SM & CEM & SI & PI & SM & CEM & SI & PI & $S M$ & CEM & SI & $\mathrm{PI}$ & SM & CEM \\
\hline 1. USA & -.38 & -.39 & -.35 & -.59 & -.37 & -.40 & -.32 & -.37 & -.47 &. .48 & -.35 & -.45 & -.55 & -.40 & -.34 & -39 \\
\hline 2. Switzerland & -.40 & -.42 & -.36 & -.61 & -.40 & -.46 & -.34 & -.39 & -.46 & -.47 & -.35 & -.44 & -.71 & -.45 & -.35 & -.42 \\
\hline 3. Canada & -.41 & -.45 & -.37 & -.63 & -.38 & -.41 & -.33 & -.37 & -.48 & -.48 & -.35 & -.45 & -.53 & -.40 & -.33 & -.39 \\
\hline 4. USA & -.39 & -41 & -.36 & -.60 & -.38 & -.41 & -.33 & -.37 & -.54 & -.55 & -.37 & -.46 & -1.00 & -.52 & -.37 & -.43 \\
\hline 5. Australia & -.40 & -.42 & -.36 & -.61 & -.37 & -.41 & -.33 & -.37 & -.50 & -.51 & -.36 & -.45 & -.61 & -.42 & -.34 & -.40 \\
\hline 6. Sweden & -.41 & -.49 & -38 & -.64 & -.38 & -.42 & -.33 & -.38 & -.45 &. .46 & -.35 & -.44 & -.79 & -.47 & -.36 & -.42 \\
\hline 7. Denmark & -.41 & -.51 & -.38 & -.64 & -.38 & -.41 & -.33 & -.37 & -.47 & -.48 & -.35 & -.44 & -.67 & -.44 & -.35 & -.41 \\
\hline 8. France & -.38 & -.39 & -.35 & .59 & -.39 & -.44 & -.34 & -.39 & -.51 & -.53 & -.36 & -.46 & -.57 & -.41 & -.34 & -.40 \\
\hline 9. Norway & -.41 & -.45 & -.37 & -.63 & -.39 & -.43 & -.34 & -.38 & -.48 & -.48 & -.35 & -.45 & -.65 &. .43 & -.35 & -.41 \\
\hline 10. UK & -.38 & -.63 & -.38 & -.65 & -.38 & -.43 & -.33 & -.38 & -.45 & -.45 & -.35 & -.44 & -.48 &. .38 & -32 & -.38 \\
\hline 11. Germany & -.41 & -.48 & -.38 & -.64 & -.39 & -.43 & -.34 & -.38 &. .49 & -.50 & -.36 & -.45 & -.70 &. .45 & -.35 & -.41 \\
\hline 12. Japan & -.39 & -.40 & -.35 & -.60 & -.41 & -.49 & -.35 & -.40 & -.46 & -.47 & -.35 &. .44 & -.53 & -.40 & -.33 & -.39 \\
\hline 13. Israel & -.41 & -.46 & -.37 & .63 & -.40 & -.46 & -.34 & -.39 & -.53 & -.55 & -.36 & -.46 & -.68 & -.44 & -.35 & -.41 \\
\hline 14. Italy & -.41 & -.45 & -.37 & -.63 & -.40 & -.46 & -.34 & -.39 & -.49 & -.50 & -.36 & -.45 & -.55 & -.40 & -.34 & -.39 \\
\hline 15. Belgium & -.40 & -.42 & -36 & -.61 & -.40 & -.46 & -.35 & -.39 & .58 &. .61 & -.37 & -.47 & -.61 & -.42 & -.34 & -.40 \\
\hline 16. Austria & -.41 & -.46 & -.37 & -.63 & -.39 & -.43 & -.33 & -.38 & -.52 & -.54 & -.36 & -.46 & -.50 & -.39 & -.33 & -.38 \\
\hline 17. Australia* & -.40 & -.43 & -.36 & -.62 & -.39 & -.43 & -.34 & -.38 & -.58 & -.61 & -.37 & -.47 & -.58 & -.41 & -.34 & -.40 \\
\hline 18. Hong Kong & -.40 & -.43 & -.37 & -.62 & -.42 & -.52 & -.36 & -.41 & -.48 &. .49 & -.36 & -.45 & -.83 & -.48 & -.36 & -.42 \\
\hline 19. Sweden * & -.41 & -.45 & -.37 & -.63 & -.38 & -.42 & -.33 & -.38 & -.47 &. .47 & -.35 & -.44 & -1.69 & -.67 & -.38 & -.44 \\
\hline 20. Netherlands & -.40 & -.42 & -.36 & -.61 & -.43 & -.54 & .36 & -.41 & -.51 & -.52 & -.36 & -.46 & -.66 & -.44 & -.35 & -.41 \\
\hline 21. Finland & -41 &. .48 & -.38 & -.64 & -.38 & -.43 & -.33 & -.38 & -.49 & .50 & -.36 & -.45 & -.66 & -.44 & -.35 & -.41 \\
\hline 22. Iceland & -.40 & -.42 & -.36 & -.62 & -39 & -.44 & -.34 & -39 &. .53 & -55 & -.36 & -.46 & -.98 & -.52 & -.37 & -.43 \\
\hline 23. UK* & -.41 & -.49 & -.38 & -.64 & -.40 & -.46 & -.34 & -.39 & -.49 & -50 & -.36 & -.45 & -1.15 & -.55 & -.37 & -.43 \\
\hline 24. Singapore & -.41 & -.48 & -.38 & -.64 & -.39 & -.44 &. .34 & -.39 & -.44 & -.44 & -.34 & -.43 & -.46 & -.37 & -.32 & -.37 \\
\hline 25. Spain & -.41 & -.44 & -.37 & -.62 & -.41 & -.48 & .35 & -.40 & -.51 & -.53 & -.36 & -.46 & -.69 &. .45 & -.35 & -.41 \\
\hline 26. Mexica & -.41 & -.45 & -.37 & -.63 & -.41 & -.49 & -.35 & -.40 & -.55 & -.57 & -.37 & -.46 & -.64 & -.43 & -35 & -.41 \\
\hline 27. Israel & -.40 & -.42 & -.36 & -.61 & -.42 & -.53 & -.36 & -.41 & -.48 & -.49 & -.36 & -.45 & -.64 & -.43 & -.35 & -.41 \\
\hline 28. Italy* & -.40 & -.42 & -.36 & -.61 & -.42 & -.51 & -.36 & -.41 & -.49 & -.50 & -.36 & -.45 & -2.14 & -.76 & -.38 & -.44 \\
\hline 29. Puerto Rico & -.41 & -.44 & -.37 & -.63 & -.38 & -.42 & -.33 & -.38 & -.51 & -52 & -.36 & -.45 & -.61 & -.42 & -.34 &. .40 \\
\hline 30. Malta & -.41 & -.45 & -.37 & -.63 & -.38 & -.42 & -.33 & -.38 & -.51 & .52 & -.36 & -.46 & -.55 & -.40 & -.34 & -.39 \\
\hline 31. Puerto Rico* & -.40 & -.42 & -.36 & -.61 & -.39 & -.44 & -.34 & -39 & -.47 & -.47 & -.35 & -.44 & -1.22 & -.57 & -.37 & -.44 \\
\hline 32. Ireland & -.40 & -.55 & -.38 & -.65 & -.41 & -.49 & -.35 &. .40 & -.51 &. .53 & -.36 & -.46 & -.88 & -.49 & -.36 & -.43 \\
\hline 33. South Africa & -.41 & -.44 & -.37 & -.62 & -.39 & -.43 & -.34 & -.38 & -56 & -59 & -.37 & -.46 & -1.13 & -.55 & -.37 & -.43 \\
\hline 34. Colombia & -.40 & -.43 & -.37 & -.62 & -.39 & -.44 & -34 & -38 & -.54 & -.56 & -.37 & -.46 & -.59 & -.42 & -34 & -.40 \\
\hline 35. Taiwan & -.41 & -.44 & -.37 & -.62 & -.46 & -.68 & -.37 & -.42 & -.46 & -.46 & -.35 & -.44 & -.78 & -.47 & -.36 & -.42 \\
\hline 36. Korea & -.41 & -.46 & -37 & -.63 & -.42 & -.50 & -.35 & -.40 & -.49 & -.50 & -.36 & -.45 & -.72 & -.45 & -.35 & -.42 \\
\hline 37. Greece & -.41 & -.45 & -.37 & -.63 & -.43 & -.54 & -.36 & -.41 & -.52 & .53 & -.36 & -.46 & -1.75 & -.68 & -.38 & -.44 \\
\hline 38. Panama & -.41 & -.44 & -.37 & -.62 & -.41 & -.48 & -.35 & -.40 & -.49 & -.50 & -.36 & -.45 & -1.75 & -.68 & -.38 & -.44 \\
\hline 39. Thuiland & -.40 & -.44 & -.37 & -.62 & -.41 & -.49 & -.35 & -.40 & -.52 & -.54 & -.36 & -.46 & -.96 & -.51 & -37 & -.43 \\
\hline 40. Sri Larka & -.41 & -.49 & -.38 & -.64 & -.41 & -.49 & -.35 & -.40 & -.56 & -.58 & -.37 & -.46 & -1.68 & -.66 & -.38 & -.44 \\
\hline 41. Thailand* & -.40 & -.43 & -.36 & -.62 & -.42 & -.51 & -.36 & -.41 & -.49 & -.50 & -.36 & -.45 & -2.14 & -.76 & -.38 & -.44 \\
\hline 42. Korea ${ }^{*}$ & -.41 & -.45 & -.37 & -.63 & -.45 & -.62 & -.37 & -.42 & -.58 & -.60 & -.37 & -.47 & -1.82 & -.69 & -.38 & -.44 \\
\hline Mean & -.40 & -.45 & -.37 & -.62 & -.40 & -.47 & -.34 & -39 & -.50 & -.51 & -.36 & -.45 & -.90 & -.49 & -.35 & -.41 \\
\hline
\end{tabular}

See notes to Table 6. 
As explained in Section 2, models (2.2) and (2.3) are nested within model (2.1). That is, when $\mu_{j}=\beta_{j}(j=1, \ldots, 8)$, then (2.1) becomes (2.2); and when $\mu_{\mathrm{j}}=0,(2.1)$ becomes (2.3). Accordingly, these restrictions can be tested by using the likelihood ratio test statistic $-2 \log \lambda=-2\left(L_{r}-L_{u}\right)$, where $L_{r}$ and $L_{u}$ are the restricted and unrestricted $\log$-likelihood values. Asymptotically, $-2 \log \lambda$ is distributed $\chi^{2}(7)$. Column 2 of Table 8 contains the data-based values of the test statistic. As the 5 percent critical value of $\chi^{2}(7)=14.07$, both models are rejected relative to (2.1).

Since the likelihood ratio test has only an asymptotic justification, its power may be influenced by the sample size. Accordingly, we proceed with a Monte Carlo procedure, whereby the data-based value of the test statistic is compared with its simulated values under the null, rather than the critical value of an asymptotic distribution (S. Selvanathan, 1987, 1993, Taylor et al., 1986, Theil et al., 1985). ${ }^{9}$ Column 5 of Table 8 gives the results from the Monte Carlo test in the form of the rank of the data-based test statistic among the 1,000 simulated values. As the rank for PI is 880 , which is less than 950 (the "critical value" of the empirical distribution at the 5 percent level), we cannot reject this model against (2.1). However, as the rank for SM is 993 , this model is rejected relative to (2.1). Note that this test procedure involves normal disturbances, which is arguable in some circumstances. We relax the normality assumption by using the bootstrap procedure to generate disturbances (Efron, 1979) and the results are shown in columns 7 and 8. As can be seen, these results are similar to those under normality.

\footnotetext{
${ }^{9}$ The Monte Carlo test involves both the unrestricted model, (2.1), and the restricted model, (2.2) or (2.3). The procedure is as follows: (1) Generate disturbances from a multivariate normal distribution with zero mean vector and covariance matrix $\hat{\Sigma}$, the mean squares and cross-products of the restricted residuals; (2) generate simulated values of the dependent variable of the restricted model by using (a) the data-based estimates of its coefficients, (b) the observed values of the independent variables, and (c) the generated disturbances; (3) estimate both the restricted and unrestricted models with the generated values of the dependent variable and the observed values of the independent variables; (4) compute the value of the likelihood ratio test statistic; (5) repeat 1,000 times steps 1-4 to generate the simulated distribution of the test statistic under the null; (6) reject the null at the 5 percent level if the observed value of the test statistic is larger than 950 simulated values.
} 
TABLE 8

TESTING THE MODELS

\begin{tabular}{|c|c|c|c|c|c|c|c|}
\hline \multirow{3}{*}{$\begin{array}{c}\text { Null } \\
\text { hypothesis } \\
\text { (SI = alternative) }\end{array}$} & \multirow{3}{*}{$\begin{array}{l}\text { Observed value of } \\
\text { likelihood ratio test } \\
\text { statistic }\end{array}$} & \multirow{2}{*}{\multicolumn{2}{|c|}{ Likelihood ratio test }} & \multicolumn{4}{|c|}{ Monte Carlo test with } \\
\hline & & & & \multicolumn{2}{|c|}{ Normal errors } & \multicolumn{2}{|c|}{ Bootstrap errors } \\
\hline & & $\begin{array}{l}\text { Critical value of } \\
\chi^{2}(7) \text { at } 5 \% \text { level }\end{array}$ & $\begin{array}{l}\text { Reject } \\
\text { the null? }\end{array}$ & $\begin{array}{l}\text { Rank of } \\
\text { test statistic }\end{array}$ & $\begin{array}{l}\text { Reject } \\
\text { the null ? }\end{array}$ & $\begin{array}{l}\text { Rank of } \\
\text { test statistic }\end{array}$ & $\begin{array}{l}\text { Reject } \\
\text { the null? }\end{array}$ \\
\hline (1) & (2) & (3) & (4) & (5) & (6) & (7) & (8) \\
\hline $\mathrm{PI}$ & 14.77 & 14.07 & Yes & 880 & No & 873 & No \\
\hline SM & 26.19 & 14.07 & Yes & 993 & Yes & 989 & Yes \\
\hline
\end{tabular}

Note: $\mathrm{SI}=$ Working's model under substitution independence; PI = Working's model under preference independence; and $S M=$ Selvanathan's model. 


\section{SIMULATION PROCEDURES}

This section sets out the methodology of the matrix approach to simulation. We use the observed data set to estimate model $j$ and provisionally regard it as the true model. The implied income and own-price elasticities are calculated and regarded as the true elasticities. Artificial data for the dependent variable are then generated from model $\mathrm{j}$, by using the estimated parameters, the observations of the independent variables and simulated disturbances. These artificial data are then used to estimate model $k$. For all possible pairs $k, j=1,2,3,4$, this procedure yields a $4 \times 4$ comparison matrix.

To describe this procedure in more detail, we write $M(k \mid j)$ to denote the situation when model $k$ is estimated given that the true model is $j$. This $M(k \mid j)$ lies behind the $(k, j)^{\text {th }}$ cell of the $4 \times 4$ comparison matrix, as shown in Table 9 . Table 10 gives the detailed simulation procedures for $M(k \mid j)$. By repeating Steps 1-9 of Table 10, with appropriate modifications for the true and the estimated models, we obtain all entries of Table 9.

\section{SIMULATION RESULTS}

Table 11 contains the biases of the income and own-price elasticity estimates from the simulation. The table is organised into ten divisions comprising the eight commodities and the two versions of the overall mean. Each division contains two $5 \times 5$ matrices corresponding to the income and own-price elasticity. Each $5 \times 5$ matrix comprises the $4 \times 4$ comparison matrix bordered with a row and column containing the means. 
TABLE 9

THE COMPARISON MATRIX

\begin{tabular}{|c|c|c|c|c|}
\hline \multirow{2}{*}{ Estimated model } & \multicolumn{4}{|c|}{ True model } \\
\hline & $j=1$ & $j=2$ & $j=3$ & $j=4$ \\
\hline$k=1$ & $\begin{array}{l}M(1 \mid 1) \text {, both the true and } \\
\text { the estimated models are } \\
\text { model }(2.1) \text {. }\end{array}$ & $\begin{array}{l}\text { M }(1 \mid 2) \text {, estimate model } \\
\text { (2.1) given that the true model } \\
\text { is model }(2.2) \text {. }\end{array}$ & $\begin{array}{l}\text { M ( } 1 \mid 3) \text {, estimate model } \\
(2.1) \text { given that the true model } \\
\text { is model }(2.3) \text {. }\end{array}$ & $\begin{array}{l}\text { M ( } 1 \mid 4) \text {, estimate model } \\
\text { (2.1) given that the true model } \\
\text { is model } \quad(2.4) \text {. A } \\
\text { transformation procedure is } \\
\text { needed. }\end{array}$ \\
\hline$k=2$ & $\begin{array}{l}\text { M ( } 2 \mid 1) \text {, estimate model } \\
(2.2) \text { given that the true model } \\
\text { is model }(2.1) \text {. }\end{array}$ & $\begin{array}{l}\mathrm{M}(2 \mid 2) \text {, both the true and } \\
\text { the estimated models are } \\
\text { model }(2.2) \text {. }\end{array}$ & $\begin{array}{l}\text { M ( } 2 \mid 3) \text {, estimate model } \\
\text { (2.2) given that the true model } \\
\text { is model }(2.3) \text {. }\end{array}$ & $\begin{array}{l}\text { M }(2 \mid 4) \text {, estimate model } \\
(2.2) \text { given that the true model } \\
\text { is model }(2.4) \text { A A } \\
\text { transformation procedure is } \\
\text { needed. }\end{array}$ \\
\hline $\mathrm{k}=3$ & $\begin{array}{l}\text { M ( } 3 \mid 1 \text { ), estimate model } \\
\text { (2.3) given that the true model } \\
\text { is model }(2.1) \text {. }\end{array}$ & $\begin{array}{l}\mathrm{M}(3 \mid 2) \text {, estimate model } \\
\text { (2.3) given that the true model } \\
\text { is model }(2.2) \text {. }\end{array}$ & $\begin{array}{l}M(3 \mid 3) \text {, both the true and } \\
\text { the estimated models are } \\
\text { model }(2.3) \text {. }\end{array}$ & $\begin{array}{l}M(3 \mid 4) \text {, estimate model } \\
(2.3) \text { given that the true model } \\
\text { is model }(2.4) \text {. A } \\
\text { transformation procedure is } \\
\text { needed. }\end{array}$ \\
\hline$k=4$ & $\begin{array}{l}\text { M ( } 4 \mid 1 \text { ), estimate model } \\
\text { (2.4) given that the true model } \\
\text { is model }(2.1) . \quad A \\
\text { transformation procedure is } \\
\text { needed. }\end{array}$ & 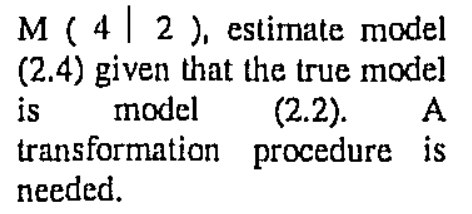 & $\begin{array}{l}\text { M ( } 4 \mid 3) \text {, estimate model } \\
\text { (2.4) given that the true model } \\
\text { is model } \quad(2.3) \text { A A } \\
\text { transformation procedure is } \\
\text { needed. }\end{array}$ & $\begin{array}{l}M(4 \mid 4) \text {, both the true and } \\
\text { the estimated models are } \\
\text { model }(2.4) \text {. }\end{array}$ \\
\hline
\end{tabular}


TABLE 10

SIMULATION PROCEDURES FOR $M(k \mid j)$

Step Description

Step $1 \quad$ Define the true model

Let the true model be model $\mathrm{j}$. Estimate model $\mathrm{j}$ with the observed data to yield estimates of the coefficient vector, $\hat{\theta}$, and the disturbance covariance matrix, $\hat{\Sigma}$. Then compute the implied income and own-price elasticities for commodity $i$ in country $c$, to be denoted by $\eta_{\mathrm{ic}}^{*}$ and $\eta_{\mathrm{iic}}^{*}$. These are interpreted as the true elasticities.

Step 2 Generate artificial data from the true model

First, generate a pseudo-normal random error vector $\varepsilon^{*}$ with zero mean and covariance matrix $\hat{\Sigma}$. Second, use (i) the estimates of the coefficients $\hat{\theta}$; (ii) the $\mathrm{X}$ matrix; and (iii) the simulated error vector $\varepsilon^{*}$ to generate artificial data on the dependent variable from model $\mathrm{j}: \mathbf{z}^{*}=\mathbf{X} \hat{\theta}+\varepsilon^{*}$.

\section{Step 3 Transform the dependent variable}

When the dependent variables differ for the true and estimated models, transform the artificial data from the true model $j, z^{*}=\left[z_{i c}^{*}\right]$, to be compatible with the estimated model $k$. For $k=4$ and $j=1,2,3$, the transformation takes the form $z_{i c}^{* *}=z_{i c}^{*} / \bar{w}_{i c}+D Q_{c}$, where $\bar{w}_{i c}$ and $D Q_{c}$ are the observed values of the budget share of good $i$ in country $c$ and the Divisia volume index for $\mathrm{c}$. For $\mathrm{j}=4$ and $\mathrm{k}=1,2,3$, the transformation takes the form $z_{i c}^{*}=\bar{w}_{i c}\left(z_{i c}^{*}-D Q_{c}\right)$. In all other cases, as the dependent variables coincide, the transformation is not needed.

Step 4 Define the estimated model

Let the estimated model be model $k$. Use $z^{*}$, or $z^{* *}=\left[z_{i c}^{* *}\right]$ if a transformation has taken place, together with the observed values of the independent variables, to estimate model $\mathrm{k}$ and compute the elasticities, to be denoted by $\eta_{\mathrm{ic}}^{\mathrm{s}}$ and $\eta_{\mathrm{iic}}^{\mathrm{s}}$. These are interpreted as the simulated elasticities. 
TABLE 10 (continued)

SIMULATION PROCEDURES FOR $M(k \mid j)$

\begin{tabular}{ll}
\hline \hline Step & Description \\
\hline Step 5 & Replication
\end{tabular}

Repeat Steps 2-4 1000 times and record the simulated elasticities, $\eta_{\mathrm{ic}}^{\mathrm{s}}$ and $\eta_{\text {iic }}^{\mathrm{s}}$, each time.

Step 6 Summarise the results over trials

Compute the biases (BIAS) and the root-mean-squared errors (RMSE) of the simulated elasticities for each commodity in each country:

$$
\begin{aligned}
& \mathrm{BLAS}_{\mathrm{ic}}=\left(\frac{1}{1000} \sum_{\mathrm{s}=1}^{1000} \eta_{\mathrm{ic}}^{\mathrm{s}}\right)-\eta_{\mathrm{ic}}^{*}, \quad \mathrm{BIAS}_{\mathrm{icc}}=\left(\frac{1}{1000} \sum_{\mathrm{s}=1}^{1000} \eta_{\mathrm{iic}}^{\mathrm{s}}\right)-\eta_{\mathrm{ic}}^{*} \\
& \operatorname{RMSE}_{\mathrm{ic}}=\sqrt{\frac{1}{1000} \sum_{\mathrm{s}=1}^{1000}\left(\eta_{\mathrm{ic}}^{\mathrm{s}}-\eta_{\mathrm{ic}}^{*}\right)^{2}}, \operatorname{RMSE}_{\mathrm{iic}}=\sqrt{\frac{1}{1000} \sum_{\mathrm{s}=1}^{1000}\left(\eta_{\mathrm{iic}}^{\mathrm{s}}-\eta_{\mathrm{iic}}^{*}\right)^{2}} .
\end{aligned}
$$

Compute the standard errors (SE) for the estimated income and own-price elasticities as:

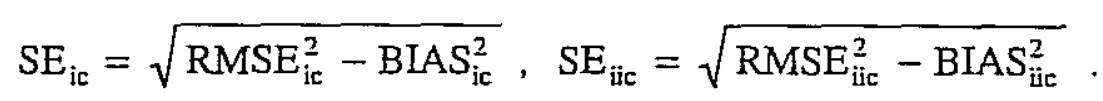

Step 7 Summarise the results over countries

Compute the means of the biases, the RMSEs and the SEs over the 42 countries:

$$
\begin{aligned}
& \mathrm{BIAS}_{\mathrm{i}}=\frac{1}{42} \sum_{\mathrm{c}=1}^{42} \mathrm{BIAS}_{\mathrm{ic}}, \quad \text { BIAS } \mathrm{S}_{\mathrm{ii}}=\frac{1}{42} \sum_{\mathrm{c}=1}^{42} \mathrm{BIAS}_{\mathrm{iic}}, \\
& \mathrm{RMSE}_{\mathrm{i}}=\frac{1}{42} \sum_{\mathrm{c}=1}^{42} \mathrm{RMSE}_{\mathrm{ic}}, \quad \mathrm{RMSE}_{\mathrm{ii}}=\frac{1}{42} \sum_{\mathrm{c}=1}^{42} \mathrm{RMSE}_{\mathrm{ic}}, \\
& \mathrm{SE}_{\mathrm{i}}=\frac{1}{42} \sum_{\mathrm{c}=1}^{42} \mathrm{SE}_{\mathrm{ic}}, \quad \mathrm{SE}_{\mathrm{ii}}=\frac{1}{42} \sum_{\mathrm{c}=1}^{42} \mathrm{SE}_{\mathrm{ic}} .
\end{aligned}
$$


TABLE 10 (continued)

SIMULATION PROCEDURES FOR $M(k \mid j)$

Step Description

Step 8 Summarise the results over commodities: Unweighted

Compute the corresponding means over the eight commodities:

$$
\begin{aligned}
& \text { BIAS }^{I}=\frac{1}{8} \sum_{i=1}^{8} \mid \text { IIAS }_{i} \mid, \quad \text { BIAS }^{\mathrm{P}}=\frac{1}{8} \sum_{\mathrm{i}=1}^{8}\left|\mathrm{BIAS}_{\mathrm{ii}}\right|, \\
& \mathrm{RMSE}^{1}=\frac{1}{8} \sum_{\mathrm{i}=1}^{8} \mathrm{RSME}_{\mathrm{i}}, \quad \operatorname{RMSE}^{\mathrm{P}}=\frac{1}{8} \sum_{\mathrm{i}=1}^{8} \operatorname{RMSE}_{\mathrm{ii}}, \\
& \mathrm{SE}^{\mathrm{I}}=\frac{1}{8} \sum_{\mathrm{i}=1}^{8} \mathrm{SE}_{\mathrm{i}}, \quad \mathrm{SE}^{\mathrm{P}}=\frac{1}{8} \sum_{\mathrm{i}=1}^{8} \mathrm{SE}_{\mathrm{ii}} .
\end{aligned}
$$

\section{Step 9 Summarise the results over commodities: Weighted}

Let $\bar{w}_{i}$ be the budget share of commodity $i$ averaged over the 42 countries; these budget shares are given in the last row of Table 1 . Compute the budget-share-weighted means as:

$$
\begin{aligned}
& \text { WBIAS }^{1}=\sum_{i=1}^{8} \bar{w}_{i} \mid \text { BIAS }_{i} \mid, \quad \text { WBIAS }^{P}=\sum_{i=1}^{8} \bar{w}_{i} \mid \text { BIAS }_{i j} \mid, \\
& \text { WRMSE }^{I}=\sum_{i=1}^{8} \bar{w}_{i} \text { RMSE }_{i}, \quad \text { WRMSE }^{P}=\sum_{i=1}^{8} \bar{w}_{i} \text { RMSE }_{i j}, \\
& \text { WSE }^{I}=\sum_{i=1}^{8} \bar{w}_{i} S_{i}, \quad \text { WSE }^{P}=\sum_{i=1}^{8} \bar{w}_{i} S E_{i i} .
\end{aligned}
$$

Note: Regarding Step 3, the dependent variable of model (2.4) is $\mathrm{Dq}_{\mathrm{ic}}$, while that of models (2.1)-(2.3) is $y_{i c}=\bar{w}_{i c}\left(D q_{i c}-D Q_{r}\right)$. Accordingly, Step 3 of the simulation procedure for $M(4 \mid 1)$, $M(4 \mid 2)$ and $M(4 \mid 3)$ transforms $y_{i c}$ into $D q_{i c}$; while this step for $M(1 \mid 4), M(2 \mid 4)$ and $M(3 \mid 4)$ transforms $D q_{i c}$ into $y_{i c}$. In the notation of Step 3, this transformation implies that $z_{i c}^{*}$ is the artificial value of $D q_{i c}$ when $k=4$ and $j=1,2,3$; and $\ddot{z_{i c}}$ is the artificial value of $y_{i c}$ when $k=1,2,3$ and $j=4$. 
Consider the leading $4 \times 4$ block of the first $5 \times 5$ matrix of Table 11 referring to the biases of the income elasticity for food. The $(k, j)^{\text {th }}$ element here refers to the bias of this elasticity associated with $M(k \mid j)$, that is, the estimates of model $\mathrm{k}$, given that the true model is $\mathrm{j}$. The elements on the main diagonal of this block refer to the biases when the estimated and true models coincide, while the offdiagonal elements are the biases when the estimated and true models differ. As the element $(1,1)$ in this block is $.04\left(\times 10^{-2}\right)$, the bias of the estimated food income elasticity is $.04 \times 10^{-2}$ when model (2.1) is both the estimated and true model. As the values of the other three diagonal elements of this block $(.14, .15$ and .03 , all $\times 10^{-2}$ ) are also small, we can safely conclude that the estimated elasticities are unbiased when the "correct" model is estimated.

We now proceed across the first row of the first block. The value of $.09\left(\times 10^{-2}\right)$ in element $(1,2)$ tells us that the bias of the estimated income elasticity for food is about $.1 \times 10^{-2}$ when model (2.1) is estimated, given that the true model is (2.2). As element $(1,3)$ is $.12\left(\times 10^{-2}\right)$, the bias of the estimated income elasticity is also approximately $.1 \times 10^{-2}$ when model (2.1) is estimated, given that the true model is $(2.3)$. Element $(1,4)$ indicates that the bias jumps to $6 \times 10^{-2}$ when model (2.1) is estimated, given that the true model is (2.4). Note that as the first element in this row is the smallest, the bias is a minimum when the true and estimated models coincide, which makes intuitive sense. This property holds in the vast majority (about 80 percent) of cases in Table $11^{10}$

Since we usually do not know the form of the true model in practice, we can eliminate the role of the true model by averaging. If each of the four alternatives is equiprobable, then an unweighted average over the four alternative true models is the

\footnotetext{
${ }^{10}$ The same is true regarding the root-mean-square errors and the standard errors, to be considered in Tables 13 and 14.
} 
appropriate way to summarise the results. Column 5 of the first $5 \times 5$ matrix gives the results for the income elasticity of food. As can be seen from the first element in this column, the mean bias when model (2.1) is estimated is $1.6 \times 10^{-2}$. As this value is also the lowest in this column for the first block, we conclude that model (2.1) performs the best in terms of the bias of the estimated income elasticity for food. The interpretation of the other results in Table 11 is exactly the same. The shaded cells indicate the lowest means and thus identify the best model for each commodity and elasticity. Column 2 of Table 12 summarises the results for the biases. As can be seen from rows $1-4$, in 88 percent of the cases regarding the income elasticities, model (2.1), Working's model under substitution independence, is the best in terms of the biases. For the price elasticities (rows 6-9 of column 2), the same model dominates 75 percent of the time. Rows 11-14 of column 2 indicate that for the income and price elasticities combined, this model is ranked first 81 percent of the time.

The RMSEs and the standard errors of the elasticities are presented in Tables 13 and 14, which are organised in the same way as Table 11 . These results are summarised in columns 3 and 4 of Table 12. On the basis of the RMSEs, model (2.1) dominates with respect to the income elasticities, while (2.3), Selvanathan's model, wins regarding the price elasticities, as well as for the income and price elasticities combined. Finally, the standard errors given in column 4 reveal that models (2.2) and (2.3) dominate on the basis of the income elasticities and price elasticities, respectively; and (2.3) wins again for the income and price elasticities combined.

\section{A LOSS-FUNCTION APPROACH}

We have considered the performance of the models on the basis of the biases, the RMSEs and the standard errors. It seems natural now to combine this information into a scalar measure by way of a loss function. 
TABLE 11

BIASES FOR INCOME AND OWN-PRICE ELASTICITIES

\begin{tabular}{|c|c|c|c|c|c|c|c|c|c|c|c|c|c|c|c|c|c|c|c|c|}
\hline \multirow{2}{*}{$\begin{array}{l}\text { True } \\
\text { model }\end{array}$} & \multicolumn{5}{|c|}{ Income elasticity } & \multicolumn{5}{|c|}{ Own-price elasticity } & \multicolumn{5}{|c|}{ Income elasticity } & \multicolumn{5}{|c|}{ Own-price elasticity } \\
\hline & $\begin{array}{l}\text { SI } \\
(1)\end{array}$ & $\begin{array}{l}\mathrm{PI} \\
(2)\end{array}$ & $\begin{array}{l}S M \\
\text { (3) }\end{array}$ & $\begin{array}{l}\text { CEM } \\
(4)\end{array}$ & $\begin{array}{c}\text { Menn } \\
(5)\end{array}$ & $\begin{array}{l}\text { SI } \\
(6)\end{array}$ & $\begin{array}{l}\text { PI } \\
\text { (7) }\end{array}$ & $\begin{array}{l}S M \\
(8)\end{array}$ & $\begin{array}{l}\text { CEM } \\
\text { (9) }\end{array}$ & $\begin{array}{c}\text { Mean } \\
(10)\end{array}$ & $\begin{array}{r}\text { SI } \\
(11)\end{array}$ & $\begin{array}{c}\text { PI } \\
(12)\end{array}$ & $\begin{array}{l}S M \\
(13)\end{array}$ & $\begin{array}{l}\text { CEM } \\
\text { (14) }\end{array}$ & $\begin{array}{c}\text { Mean } \\
(15)\end{array}$ & $\begin{array}{r}\text { SI } \\
(16)\end{array}$ & $\begin{array}{r}\text { PI } \\
(17)\end{array}$ & $\begin{array}{l}S M \\
(18)\end{array}$ & $\begin{array}{r}\text { CEM } \\
\text { (19) }\end{array}$ & $\begin{array}{r}\text { Mean } \\
(20)\end{array}$ \\
\hline & \multicolumn{10}{|c|}{ Food } & \multicolumn{10}{|c|}{ Clothing } \\
\hline 1. SI & .04 & .09 & .12 & 6.03 & 4 & .74 & .93 & .97 & 7.43 & 2.52 & .35 & .13 & .42 & .10 & M & .12 & .19 & .25 & 2.72 & wy \\
\hline 2. PI & 5.83 & .14 & .89 & 5.31 & 3.04 & 1.26 & .05 & 6.53 & 3.96 & 2.95 & .10 & .05 & 3.37 & 2.74 & 1.57 & 1.31 & .08 & 32 & 2.44 & 1.04 \\
\hline 3. $S M$ & .17 & .39 & .15 & 6.23 & 1.74 & 3.67 & 5.71 & .13 & 11.93 & 5.36 & 4.10 & 2.11 & .19 & 2.70 & 2.28 & 11.36 & 6.01 & .19 & 4.80 & 5.59 \\
\hline 4. CEM & 6.37 & 6.71 & 6.73 & .03 & 4.96 & 4.24 & 1.87 & 1.17 & .28 & $\begin{array}{l}m 189 \\
H_{10}\end{array}$ & .12 & .34 & 1.07 & .39 & .48 & 3.41 & 3.36 & 1.83 & .09 & 2.17 \\
\hline \multirow[t]{2}{*}{ 5. Mean } & 3.10 & 1.83 & 1.97 & 4.40 & 2.83 & 2.48 & 2.14 & 2.20 & 5.90 & 3.18 & 1.17 & .66 & 1.26 & 1.48 & 1.14 & 4.05 & 2.41 & .65 & 2.51 & 2.41 \\
\hline & \multicolumn{10}{|c|}{ Housing } & \multicolumn{10}{|c|}{ Durables } \\
\hline 6. SI & .09 & .15 & .05 & 6.77 & 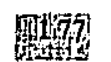 & .37 & .19 & .54 & .02 & the & .18 & .02 & .00 & 5.07 & 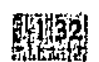 & .11 & .36 & .54 & 1.95 & 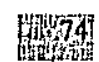 \\
\hline 7. PI & 33.65 & .13 & 7.66 & 13.15 & 13.65 & 30.91 & .00 & 9.62 & 6.12 & 11.66 & 24.83 & .04 & 8.72 & 11.42 & $\begin{array}{l}\text { Futhintitut } \\
11.25\end{array}$ & 17.80 & .06 & 13.96 & 15.79 & 11.90 \\
\hline B. SM & 2.29 & 1.00 & .31 & 7.97 & 2.89 & 52.74 & 19.24 & .18 & 21,01 & 23.29 & 8.08 & 3.93 & .19 & 9.00 & 5.30 & 52.82 & 27.02 & .19 & 38.19 & 29.56 \\
\hline 9. CEM & 11.52 & 7.19 & 2.47 & .17 & 5.34 & 1.54 & 9.56 & 12.20 & .31 & 5.90 & 7.08 & 7.26 & 6.00 & .05 & 5.10 & 7.57 & 6.68 & 6.20 & .33 & 5.20 \\
\hline \multirow[t]{2}{*}{ 10. Mean } & 11.89 & 2.12 & 2.62 & 7.02 & 5.91 & 21.39 & 7.25 & 5.64 & 6.87 & 10.28 & 10.04 & 2.81 & 3.73 & 6.39 & 5.74 & 19.58 & 8.53 & 5.22 & 14.07 & 11.85 \\
\hline & \multicolumn{10}{|c|}{ Medicine } & \multicolumn{10}{|c|}{ Transport } \\
\hline 11.SI & .46 & .27 & .07 & 11.98 & 㼛5 & .93 & 1.01 & 1.13 & 13.08 & Wtipd & .55 & .62 & .31 & 29.90 & 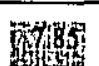 & .41 & .04 & .18 & 3.40 & Mot \\
\hline 12. PI & 3.43 & .14 & 1.63 & 10.01 & 3.80 & 6.97 & .01 & .45 & 10.56 & $4.50^{\circ}$ & 5.12 & .75 & 4.80 & 36.73 & 11.85 & 9.89 & .36 & .61 & 5.95 & 4.20 \\
\hline 13. SM & 1.36 & .61 & .01 & 11.23 & 3.30 & 1.52 & 2.23 & .20 & 14.25 & 4.55 & .64 & .21 & .95 & 30.21 & 8.00 & .90 & 6.77 & .19 & 5.71 & 3.39 \\
\hline 14. CEM & 27.76 & 27.83 & 26.30 & .70 & 20.65 & 29.92 & 29.45 & 27.80 & .61 & 21.95 & 35.22 & 36.39 & 34.56 & .91 & 26.77 & 15.47 & 12.56 & 11.12 & .94 & 10.02 \\
\hline 15. Metn & 8.25 & 7.21 & 7.00 & 8.48 & 7.74 & 9.84 & 8.18 & 7.40 & 9.63 & 8.76 & 10.38 & 9.49 & 10.16 & 24.44 & 13.62 & 6.67 & 4.93 & 3.03 & 4.00 & 4.66 \\
\hline
\end{tabular}


TABLE 11 (continued)

BIASES FOR INCOME AND OWN-PRICE ELASTICITIES

\begin{tabular}{|c|c|c|c|c|c|c|c|c|c|c|c|c|c|c|c|c|c|c|c|c|}
\hline \multirow[b]{2}{*}{ model } & \multicolumn{5}{|c|}{ Income elasticity } & \multicolumn{5}{|c|}{ Own-price elasticity } & \multicolumn{5}{|c|}{ Income elasticity } & \multicolumn{5}{|c|}{ Own-price elasticity } \\
\hline & $\begin{array}{l}\text { SI } \\
\text { (1) }\end{array}$ & $\begin{array}{l}\text { PI } \\
\text { (2) }\end{array}$ & $\begin{array}{l}S M \\
\text { (3) }\end{array}$ & $\begin{array}{c}\text { CEM } \\
\text { (4) }\end{array}$ & $\begin{array}{c}\text { Mean } \\
(5)\end{array}$ & $\begin{array}{l}S I \\
(6)\end{array}$ & $\begin{array}{l}\text { PI } \\
(7)\end{array}$ & $\begin{array}{l}S M \\
(8)\end{array}$ & $\begin{array}{c}\text { CEM } \\
(9)\end{array}$ & $\begin{array}{l}\text { Mean } \\
(10)\end{array}$ & $\begin{array}{c}S I \\
\text { (11) }\end{array}$ & $\begin{array}{l}\text { Pl } \\
(12)\end{array}$ & $\begin{array}{l}\text { SM } \\
\text { (13) }\end{array}$ & $\begin{array}{l}\text { CEM } \\
\text { (14) }\end{array}$ & $\begin{array}{c}\text { Mean } \\
(15)\end{array}$ & $\begin{array}{c}\text { SI } \\
(16)\end{array}$ & $\begin{array}{c}\text { PI } \\
\text { (17) }\end{array}$ & $\begin{array}{l}S M \\
(18)\end{array}$ & $\begin{array}{l}\text { CEM } \\
(19)\end{array}$ & $\begin{array}{c}\text { Mean } \\
(20)\end{array}$ \\
\hline & \multicolumn{10}{|c|}{ Recreation } & \multicolumn{10}{|c|}{ Other } \\
\hline 16. SI & .10 & .00 & .07 & 9.59 & 424 & .58 & .80 & .94 & 2.65 & 1024 & .40 & .13 & .50 & 17.51 & 4.64 & .45 & .73 & 2.02 & 105.06 & 27.07 \\
\hline 17. PI & 6.29 & .23 & 1.09 & 5.20 & 3.20 & 4.78 & .13 & 5.83 & 12.47 & 5.80 & 16.77 & .52 & 1.06 & 44.31 & 15.67 & 35.00 & .27 & 2.53 & 25.14 & 15.74 \\
\hline 18. SM & 1.80 & 1.83 & .08 & 8.87 & 3.15 & 9.52 & 9.82 & .19 & 1.72 & 5.31 & 5.24 & .92 & .06 & 23.25 & 7.37 & 49.96 & 7.83 & .19 & 4.79 & 4569 \\
\hline 19. CEM & 9.53 & 9.26 & 9.26 & .71 & 7.19 & 7.42 & 9.67 & 9.94 & .35 & 6.85 & 7.41 & 5.99 & 4.66 & .38 & 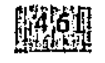 & 60.11 & 44.50 & 45.11 & .67 & 37.60 \\
\hline \multirow[t]{2}{*}{ 20. Mean } & 4.43 & 2.83 & 2.63 & 6.09 & 3.99 & 5.58 & 5.11 & 4.23 & 4.30 & 4.80 & 7.46 & 1.89 & 1.57 & 21.36 & 8.07 & 36.38 & 13.33 & 12.46 & 33.92 & 24.02 \\
\hline & \multicolumn{10}{|c|}{ Mean-Unweighted } & \multicolumn{10}{|c|}{ Mean-Weighted } \\
\hline 21. SI & .27 & .18 & .19 & 10,87 & Wab & .46 & .53 & .82 & 17.04 & 474 & .20 & .16 & .18 & 9.68 & (15) & .51 & .58 & .81 & 13.23 & 14 \\
\hline 22. PI & 12.00 & .25 & 3.65 & 16.11 & 8.00 & 13.49 & .12 & 4.98 & 10.30 & 7.22 & 11.70 & .23 & 3.25 & 13.76 & 7.24 & 11.35 & .11 & 5.64 & 8.00 & 6.28 \\
\hline 23. SM & 2.96 & 1.38 & .24 & 12.43 & 4,25 & 22.81 & 10.58 & .18 & 12.80 & 11.59 & 2.13 & 1.06 & .25 & 10.92 & 3.59 & 19.50 & 9.84 & .17 & 12.73 & 10.56 \\
\hline 24. CEM & 13.13 & 12.62 & 11.38 & .42 & 9.39 & 16.21 & 14.71 & 14.42 & .45 & 11.45 & 11.16 & 10.66 & 9.54 & .30 & 7.92 & 11.47 & 10.23 & 10.02 & .40 & 8.03 \\
\hline 25. Mean & 7.09 & 3.61 & 3.87 & 9.96 & 6.13 & 13.24 & 6.49 & 5.10 & 10.15 & 8.74 & 6.30 & 3.03 & 3.31 & 8.67 & 5.32 & 10.71 & 5.19 & 4.16 & 8.59 & 7.16 \\
\hline
\end{tabular}

Notes: 1 . SI = Working's model under substitution independence, PI = Working's model under preference independence, SM $=$ Selvanathan's model, and CEM = Constant elasticity model.

2. All entries are to be divided by 100 .

3. Shaded cells indicate the minimum of the relevant four entries of the column. 
TABLE 12

FIRST SUMMARY OF PERFORMANCE OF MODELS

(Percentage of cases when model is ranked first)

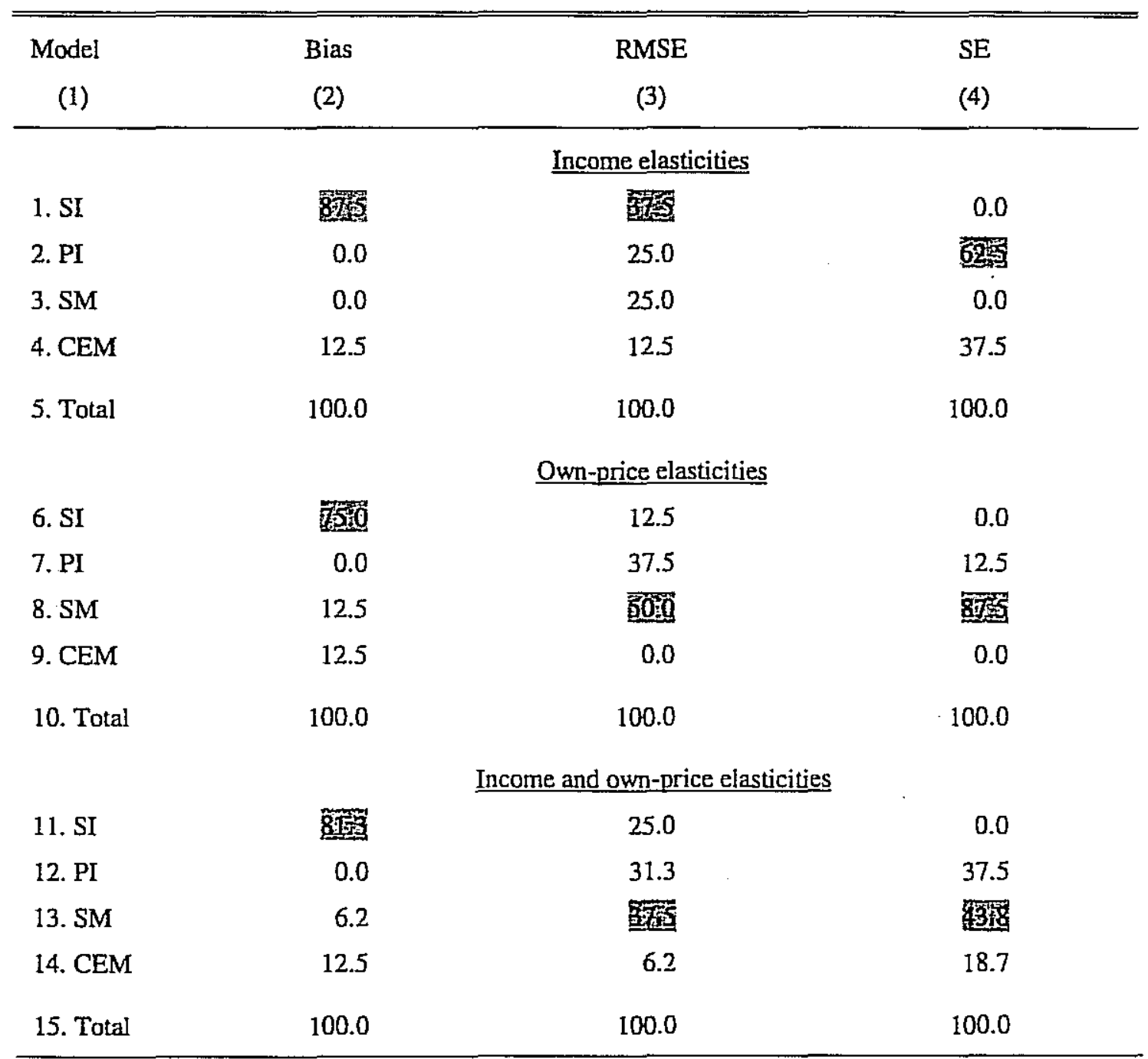

Notes: $1 . \mathrm{SI}=$ Working's model under substitution independence, $\mathrm{PI}=$ Working's model under preference independence, $\mathrm{SM}=$ Selvanathan's model, and CEM = Constant elasticity model.

2. Shaded cells indicate the maximum of the relevant four entries of the column. 
TABLE 13

ROOT-MEAN-SQUARE ERRORS FOR INCOME AND OWN-PRICE ELASTICITIES

\begin{tabular}{|c|c|c|c|c|c|c|c|c|c|c|c|c|c|c|c|c|c|c|c|c|}
\hline \multirow{2}{*}{$\begin{array}{l}\text { True } \\
\text { model }\end{array}$} & \multicolumn{5}{|c|}{ Income elasticity } & \multicolumn{5}{|c|}{ Own-price elasticity } & \multicolumn{5}{|c|}{ Income elasticity } & \multicolumn{5}{|c|}{ Own-price elasticity } \\
\hline & $\begin{array}{l}\text { SI } \\
\text { (1) }\end{array}$ & $\begin{array}{l}\text { PI } \\
(2)\end{array}$ & $\begin{array}{l}S M \\
(3)\end{array}$ & $\begin{array}{l}\text { CEM } \\
\text { (4) }\end{array}$ & $\begin{array}{c}\text { Mean } \\
\text { (5) }\end{array}$ & $\begin{array}{l}\text { SI } \\
(6)\end{array}$ & $\begin{array}{l}\text { PI } \\
\text { (7) }\end{array}$ & $\begin{array}{l}S M \\
(8)\end{array}$ & $\begin{array}{l}\text { CEM } \\
\text { (9) }\end{array}$ & $\begin{array}{c}\text { Mean } \\
(10)\end{array}$ & $\begin{array}{c}S I \\
(11)\end{array}$ & $\begin{array}{c}\text { PI } \\
\text { (12) }\end{array}$ & $\begin{array}{l}S M \\
\text { (13) }\end{array}$ & $\begin{array}{l}\text { CEM } \\
(14)\end{array}$ & $\begin{array}{c}\text { Mean } \\
(15)\end{array}$ & $\begin{array}{c}\text { SI } \\
(16)\end{array}$ & $\begin{array}{c}\text { PI } \\
(17)\end{array}$ & $\begin{array}{l}S M \\
(18)\end{array}$ & $\begin{array}{l}\text { CEM } \\
(19)\end{array}$ & $\begin{array}{c}\text { Mein } \\
(20)\end{array}$ \\
\hline & \multicolumn{10}{|c|}{ Food } & \multicolumn{10}{|c|}{ Clothing } \\
\hline 1. SI & 7.96 & 7.76 & 7.51 & 11.01 & the & 9.93 & 11.20 & 10.88 & 15.07 & 11.77 & 16.36 & 16.55 & 16.78 & 20.56 & 17.56 & 12.16 & 12.59 & 12.75 & 12.79 & 12.57 \\
\hline 2. PI & 10.70 & 7.60 & 7.60 & 10.80 & 9.18 & 5.06 & 3.95 & 7.64 & 6.50 & 45 & 16.11 & 15.81 & 16.69 & 18.92 & Mfis & 8.70 & 7.98 & 7.31 & 8.50 & $8[2]$ \\
\hline 3. SM & 7.97 & 7.70 & 7.56 & 11.09 & 8.58 & 6.26 & 7.32 & 4.12 & 12.75 & 7.61 & 16.68 & 16.44 & 16.48 & 20.70 & 17.58 & 13.35 & 8.67 & 5.85 & 8.01 & 8.97 \\
\hline 4. CEM & 11.80 & 11.52 & 11.70 & 6.62 & 10.41 & 11.48 & 10.11 & 9.20 & 7.26 & 9.51 & 17.78 & 17.88 & 17.67 & 17.56 & 17.72 & 15.00 & 14.21 & 13.39 & 11.75 & 13.59 \\
\hline \multirow[t]{2}{*}{ 5. Mean } & 9.61 & 8.65 & 8.59 & 9.88 & 9.18 & 8.18 & 8.15 & 7.96 & 10.40 & 8.67 & 16.73 & 16.67 & 16.91 & 19.44 & 17.44 & 12.30 & 10.86 & 9.83 & 10.26 & 10.81 \\
\hline & \multicolumn{10}{|c|}{ Housing } & \multicolumn{10}{|c|}{ Durables } \\
\hline 6. SI & 12.68 & 13.96 & 14.96 & 18.41 & mos & 9.80 & 11.04 & 11.67 & 15.16 & 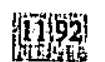 & 17.30 & 17.64 & 18.91 & 28.12 & 20 & 18.48 & 20.01 & 22.16 & 22.83 & 20.87 \\
\hline 7. PI & 36.16 & 12.18 & 15.65 & 19.98 & 20.99 & 31.38 & 5.47 & 11.28 & 10.63 & 14.69 & 29.57 & 15.79 & 19.64 & 27.80 & 23.20 & 21.64 & 9.96 & 17.01 & 22.40 & 1755 \\
\hline 8. SM & 12.81 & 13.90 & 14.90 & 18.91 & 15.13 & 53.18 & 20.16 & 5.49 & 21.85 & 25.17 & 18.60 & 17.72 & 18.54 & 28.82 & 20.92 & 53.36 & 27.87 & 5.96 & 38.75 & 31.49 \\
\hline 9. CEM & 18.14 & 20.82 & 17.63 & 13.26 & 17.46 & 23.53 & 18.20 & 19.22 & 6.11 & 16.77 & 31.46 & 37.12 & 34.75 & 17.07 & 30.10 & 34.69 & 28.08 & 25.39 & 14.91 & 25.77 \\
\hline \multirow[t]{2}{*}{ 10. Mean } & 19.95 & 15.22 & 15.79 & 17.64 & 17.15 & 29.47 & 13.72 & 11.92 & 13.44 & 17.14 & 24.23 & 22.07 & 22.96 & 25.45 & 23.68 & 32.04 & 21.48 & 17.63 & 24.72 & 23.97 \\
\hline & \multicolumn{10}{|c|}{ Medicine } & \multicolumn{10}{|c|}{ Transport } \\
\hline 11. SI & 30.71 & 30.82 & 30.23 & 31,23 & 30.75 & 24.07 & 25.22 & 26.03 & 26.00 & 25.33 & 17.98 & 17.87 & 18.11 & 39.12 & 23.27 & 17.60 & 18.98 & 20.68 & 21.48 & 19.69 \\
\hline 12. PI & 28.96 & 28.02 & 28.96 & 28.56 & {$\left[\begin{array}{l}38 \\
8\end{array}\right.$} & 15.52 & 12.81 & 11.74 & 17.83 & 14.48 & 18.58 & 16.83 & 17.99 & 43.70 & 24.28 & 13.23 & 7.91 & 7.52 & 10.36 & 9.76 \\
\hline 13. SM & 30.82 & 30.77 & 30.57 & 30.71 & 30.72 & 7.37 & 7.24 & 6.15 & 15.76 & 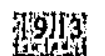 & 17.91 & 17.74 & 17.94 & 39.17 & $25 y$ & 6.79 & 9.31 & 5.74 & 8.50 & Trips \\
\hline 14. CEM & 47.26 & 47.26 & 46.17 & 20.25 & 40.24 & 40.84 & 40.72 & 39.23 & 13.05 & 33.46 & 41.75 & 42.99 & 41.33 & 21.53 & 36.90 & 28.68 & 27.02 & 25.92 & 22.85 & 26.12 \\
\hline 15. Mean & 34.44 & 34.22 & 33.98 & 27.69 & 32.58 & 21.95 & 21.50 & 20.79 & 18.16 & 20.60 & 24.06 & 23.86 & 23.84 & 35.88 & 26.91 & 16.58 & 15.81 & 14.97 & 15.80 & 15.79 \\
\hline
\end{tabular}




$$
\begin{aligned}
& \because \cdots \\
& \text { TABLE.13 (continued) }
\end{aligned}
$$

\begin{tabular}{|c|c|c|c|c|c|c|c|c|c|c|c|c|c|c|c|c|c|c|c|c|}
\hline \multirow{2}{*}{$\begin{array}{l}\text { True } \\
\text { model }\end{array}$} & \multicolumn{4}{|c|}{ Income elasticity } & \multirow{2}{*}{$\frac{}{\text { Mean }}$} & \multicolumn{5}{|c|}{ Own-price elasticity } & \multicolumn{5}{|c|}{ Income elasticity } & \multicolumn{5}{|c|}{ Own-price elasticity } \\
\hline & $\begin{array}{l}\text { SI } \\
\text { (1) }\end{array}$ & $\begin{array}{l}\text { PI } \\
\text { (2) }\end{array}$ & $\begin{array}{l}S M \\
(3)\end{array}$ & $\begin{array}{l}\text { CEM } \\
\text { (4) }\end{array}$ & & $\begin{array}{l}\text { SI } \\
\text { (6) }\end{array}$ & $\begin{array}{l}\text { PI } \\
(7)\end{array}$ & $\begin{array}{l}\text { SM } \\
(8)\end{array}$ & $\begin{array}{l}\text { CEM } \\
\text { (9) }\end{array}$ & $\begin{array}{l}\text { Mean } \\
(10)\end{array}$ & $\begin{array}{l}\text { Si } \\
\text { (11) }\end{array}$ & $\begin{array}{l}\text { PI } \\
(12)\end{array}$ & $\begin{array}{l}\text { SM } \\
\text { (13) }\end{array}$ & $\begin{array}{l}\text { CEM } \\
\text { (14) }\end{array}$ & $\begin{array}{c}\text { Mean } \\
\text { (15) }\end{array}$ & $\begin{array}{l}\text { SI } \\
(16)\end{array}$ & $\begin{array}{l}\mathrm{PI} \\
(17)\end{array}$ & $\begin{array}{l}S M \\
(18)\end{array}$ & $\begin{array}{l}\text { CEM } \\
(19)\end{array}$ & $\begin{array}{l}\text { Mean } \\
(20)\end{array}$ \\
\hline & \multicolumn{10}{|c|}{ Recreation } & \multicolumn{10}{|c|}{ Other } \\
\hline 16. SI & 16.46 & 16.84 & 17.12 & 20.82 & 17.81 & 14.99 & 15.18 & 15.20 & 15.20 & 15.14 & 31.32 & 31.77 & 31.81 & 82.02 & 44.23 & 41.71 & 49.82 & 49.99 & 146.54 & 72.02 \\
\hline 17. PI & 18.19 & 16.09 & 16.92 & 19.36 & 17.64 & 10.15 & 8.64 & 10.34 & 15.36 & 11.12 & 34.25 & 28.81 & 29.88 & 89.83 & 45.69 & 38.73 & 13.53 & 12.84 & 44.05 & 27.29 \\
\hline 18. SM & 16.57 & 16.66 & 16.94 & 20.22 & mpo & 11.92 & 11.76 & 6.00 & 6.80 & 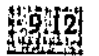 & 31.19 & 31.21 & 31.45 & 82.92 & 44.19 & 50.74 & 10.94 & 5.91 & 8.05 & Teg \\
\hline 19. CEM & 20.39 & 19.95 & 20.49 & 14.36 & 18.80 & 17.39 & 18.69 & 18.57 & 11.54 & 16.55 & 40.81 & 44.08 & $42.2 \mathrm{~B}$ & 18.87 & 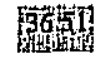 & 98.52 & 81.20 & 80.70 & 23.10 & 70.88 \\
\hline \multirow[t]{2}{*}{ 20. Menn } & 17.90 & 17.39 & 17.87 & 18.69 & 17.96 & 13.61 & 13.57 & 12.53 & 12.23 & 12.98 & 34.39 & 33.97 & 33.86 & 68.41 & 42.66 & 57.43 & 38,87 & 37.36 & 55.44 & 47.27 \\
\hline & \multicolumn{10}{|c|}{ Menn-Unweighted } & \multicolumn{10}{|c|}{ Mean-Weighted } \\
\hline $21 . \mathrm{SI}$ & 18.85 & 19.15 & 19.43 & 31.41 & 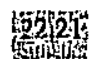 & 18.59 & 20.50 & 21.17 & 34.38 & 23.66 & 14.98 & 15.21 & 15.41 & 25.19 & 49 & 15.38 & 17.10 & 17.50 & 27.90 & 19.47 \\
\hline 22. PI & 24.07 & 17.64 & 19.17 & 32.37 & 23.31 & 18.05 & 8.78 & 10.71 & 16.95 & 1362 & 20.78 & 14.11 & 15.34 & 26.12 & 19.09 & 15.27 & 7.04 & 9.70 & 13.34 & Math \\
\hline 23. SM & 19.07 & 19.02 & 19.30 & 31.57 & 22.24 & 25.37 & 12.91 & 5.65 & 15.06 & 14.75 & 15.13 & 15.09 & 15.31 & 25.38 & 17.73 & 21.91 & 11.81 & 5.20 & 14.53 & 13.36 \\
\hline 24. CEM & 28.67 & 30.20 & $29.0 \mathrm{i}$ & 16.19 & 26.02 & 33.77 & 29.78 & 28.95 & 13.82 & 26.58 & 22.96 & 24.09 & 23.12 & 13.37 & 20.89 & 26.57 & 23.11 & 22.40 & 11.78 & 20.97 \\
\hline 25. Mean & 22.67 & 21.50 & 21.73 & 27.89 & 23.44 & 23.95 & 17.99 & 16.62 & 20.05 & 19.65 & 18.46 & 17.13 & 17.30 & 22.52 & 18.85 & 19.78 & 14.77 & 13.70 & 16.89 & 16.28 \\
\hline
\end{tabular}

$\therefore$ ROOTT-MEAN-SQUARE ERRORS FOR INCOME AND OWN-PRICE ELASTICITIES

See notes to Table 11 . 
TABLE 14

STANDARD ERRORS FOR INCOME AND OWN-PRICE ELASTICITIES

\begin{tabular}{|c|c|c|c|c|c|c|c|c|c|c|c|c|c|c|c|c|c|c|c|c|}
\hline \multirow{2}{*}{$\begin{array}{l}\text { True } \\
\text { model }\end{array}$} & \multicolumn{5}{|c|}{ Income elasticity } & \multicolumn{5}{|c|}{ Own-price elasticity } & \multicolumn{5}{|c|}{ Income elasticity } & \multicolumn{5}{|c|}{ Own-price elasticity } \\
\hline & $\begin{array}{l}\text { SI } \\
\text { (1) }\end{array}$ & $\begin{array}{l}\text { PI } \\
\text { (2) }\end{array}$ & $\begin{array}{l}S M \\
\text { (3) }\end{array}$. & $\begin{array}{c}\text { CEM } \\
\text { (4) }\end{array}$ & $\begin{array}{l}\text { Mean } \\
\text { (5) }\end{array}$ & $\begin{array}{l}\text { SI } \\
(6)\end{array}$ & $\begin{array}{l}\text { PI } \\
\text { (7) }\end{array}$ & $\begin{array}{l}S M \\
(8)\end{array}$ & $\begin{array}{c}\text { CEM } \\
(9)\end{array}$ & $\begin{array}{c}\text { Mean } \\
(10)\end{array}$ & $\begin{array}{c}\text { SI } \\
\text { (11) }\end{array}$ & $\begin{array}{c}\text { PI } \\
\text { (12) }\end{array}$ & $\begin{array}{l}\text { SM } \\
\text { (13) }\end{array}$ & $\begin{array}{l}\text { CEM } \\
(14)\end{array}$ & $\begin{array}{c}\text { Mean } \\
\text { (15) }\end{array}$ & $\begin{array}{c}\text { SI } \\
(16)\end{array}$ & $\begin{array}{c}\text { PI } \\
\text { (17) }\end{array}$ & $\begin{array}{l}S M \\
(18)\end{array}$ & $\begin{array}{l}\text { CEM } \\
(19)\end{array}$ & $\begin{array}{c}\text { Mean } \\
(20)\end{array}$ \\
\hline & \multicolumn{10}{|c|}{ Food } & \multicolumn{10}{|c|}{ Clothing } \\
\hline 1. SI & 7.96 & 7.76 & 7.51 & 7.19 & 7.61 & 9.91 & 11.16 & 10.84 & 12.16 & 11.02 & 16.35 & 16.55 & 16.78 & 20.10 & 17.45 & 12.16 & 12.58 & 12.73 & 12.49 & 12.49 \\
\hline 2. PI & 8.97 & 7.60 & 7.55 & 7.40 & 7.88 & 4.89 & 3.95 & 3.81 & 4.52 & 7429 & 16.11 & 15.81 & 16.35 & 18.25 & $y_{0}$ & 8.57 & 7.98 & 7,24 & 7.98 & 7.94 \\
\hline 3. SM & 7.97 & 7.69 & 7.56 & .7 .17 & 7.60 & 4.81 & 4.29 & 4.12 & 4.52 & 4.44 & 16.17 & 16.30 & 16.48 & 20.08 & 17.26 & 6.83 & 6.09 & 5.85 & 6.41 & 7630 \\
\hline 4. CEM & 8.15 & 7.86 & 7.72 & 6.62 & 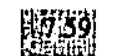 & 10.52 & 9.66 & 9.13 & 7.26 & 9.14 & 17.07 & 17.10 & 17.22 & 17.56 & 17.24 & 14.34 & 13.67 & 13.27 & 11.75 & 13.26 \\
\hline \multirow[t]{2}{*}{ 5. Mean } & 8.22 & 7.66 & 7.52 & 7.00 & 7,60 & 8.80 & 8.46 & 8.19 & 8.12 & 8.39 & 16.44 & 16.38 & 16.68 & 19.01 & 17.12 & 10.95 & 10.56 & 10.31 & 10.04 & 10.46 \\
\hline & \multicolumn{10}{|c|}{ Housing } & \multicolumn{10}{|c|}{ Durables } \\
\hline 6. SI & 12.68 & 13.96 & 14.96 & 16.13 & 14.43 & 9.79 & 11.03 & 11.64 & 11.36 & 10.96 & 17.30 & 17.64 & 18.91 & 19.33 & 18.30 & 18.48 & 20.00 & 22.13 & 18.99 & 19.90 \\
\hline 7. PI & 13.23 & 12.18 & 13.65 & 14.27 & 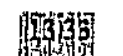 & 5.36 & 5.47 & 5.88 & 6.96 & 5.92 & 16.07 & 15.79 & 17.59 & 16.79 & $16{ }^{16}$ & 12.03 & 9.96 & 9.33 & 10.89 & 10.55 \\
\hline 8. SM & 12.61 & 13.86 & 14.89 & 16.21 & 14.39 & 6.41 & 5.72 & 5.49 & 6.02 & 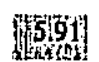 & 16.76 & 17.27 & 18.54 & 19.20 & 17.94 & 6.96 & 6.20 & 5.96 & 6.53 & 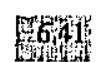 \\
\hline 9. CEM & 12.31 & 13.57 & 14.58 & 13.26 & 13.43 & 14.51 & 14.41 & 14.86 & 6.10 & 12.47 & 18.68 & 19.27 & 20.72 & 17.07 & 18.94 & 24.87 & 23.87 & 24.62 & 14.90 & 22.07 \\
\hline \multirow[t]{2}{*}{ 10. Mean } & 12.71 & 13.39 & 14.52 & 14.97 & 13.90 & 9.02 & 9.16 & 9.47 & 7.61 & 8.81 & 17.20 & 17.49 & 18.94 & 18.10 & 17.93 & 15.59 & 15.01 & 15.51 & 12.83 & 14.73 \\
\hline & \multicolumn{10}{|c|}{ Medicine } & \multicolumn{10}{|c|}{ Transport } \\
\hline $11 . S I$ & 30.71 & 30.82 & 30.23 & 28.52 & 30.07 & 24.04 & 25.19 & 25.97 & 22.23 & 24.36 & 17.97 & 17.86 & 18.11 & 22.77 & 19.18 & 17.59 & $18.9 B$ & 20.66 & 20.78 & 19.50 \\
\hline 12. PI & 28.76 & 28.02 & 28.92 & 26.40 & 2808 & $\cdot 13.85$ & $12.8 \mathrm{i}$ & 11.50 & 12.57 & 12,68 & 17.87 & 16.82 & 17.34 & 21.81 & 48 & 8.78 & 7.90 & 7.30 & 8.47 & 8.11 \\
\hline 13. SM & 30.79 & 30.76 & 30.57 & 28.26 & 30.10 & 7.18 & 6.41 & 6.15 & 6.74 & 䜊 & 17.90 & 17.74 & 17.91 & 22.51 & 19.02 & 6.70 & 5.98 & 5.74 & 6.29 & 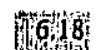 \\
\hline 14. CEM & 37.74 & $37.77^{\circ}$ & 37.53 & $20.24^{\circ}$ & $33.32^{\prime \prime}$ & 27.79 & $\cdot 28.01$ & 27.68 & 13.04 & 24.13 & 21.68 & 21.50 & 21.72 & 21.51 & 21.60 & 24.15 & 23.62 & 23.41 & 22.83 & 23.50 \\
\hline 15. Mean & 32.00 & 31.84 & 31.81 & 25.86 & 30.38 & 18.22 & 18.11 & 17.83 & 13.65 & 16.95 & 18.86 & 18.48 & 18.77 & 22.15 & 19.56 & 14.31 & 14.12 & 14.28 & 14.59 & 14.32 \\
\hline
\end{tabular}


TABLE 14 (continued)

STANDARD ERRORS FOR INCOME AND OWN-PRICE ELASTICITIES

\begin{tabular}{|c|c|c|c|c|c|c|c|c|c|c|c|c|c|c|c|c|c|c|c|c|}
\hline \multirow[b]{2}{*}{$\begin{array}{l}\text { Estimated } \\
\text { model }\end{array}$} & \multicolumn{5}{|c|}{ Income elasticity } & \multicolumn{5}{|c|}{ Own-price elasticity } & \multicolumn{5}{|c|}{ Income elasticity } & \multicolumn{5}{|c|}{ Own-price elasticity } \\
\hline & $\begin{array}{l}\text { SI } \\
\text { (1) }\end{array}$ & $\begin{array}{l}\text { PI } \\
\text { (2) }\end{array}$ & $\begin{array}{l}S M \\
(3)\end{array}$ & $\begin{array}{c}\text { CEM } \\
\text { (4) }\end{array}$ & $\begin{array}{c}\text { Mean } \\
\text { (5) }\end{array}$ & $\begin{array}{l}\text { SI } \\
(6)\end{array}$ & $\begin{array}{l}\text { PI } \\
\text { (7) }\end{array}$ & $\begin{array}{l}\text { SM } \\
(8)\end{array}$ & $\begin{array}{c}\text { CEM } \\
\text { (9) }\end{array}$ & $\begin{array}{c}\text { Mean } \\
(10)\end{array}$ & $\begin{array}{c}\text { SI } \\
\text { (11) }\end{array}$ & $\begin{array}{l}\text { PI } \\
(12)\end{array}$ & $\begin{array}{l}S M \\
(13)\end{array}$ & $\begin{array}{l}\text { CEM } \\
\text { (14) }\end{array}$ & $\begin{array}{c}\text { Mean } \\
(15)\end{array}$ & $\begin{array}{c}\text { SI } \\
\text { (16) }\end{array}$ & $\begin{array}{c}\text { PI } \\
\text { (17) }\end{array}$ & $\begin{array}{l}S M \\
(18)\end{array}$ & $\begin{array}{c}\text { CEM } \\
(19)\end{array}$ & $\begin{array}{c}\text { Menn } \\
(20)\end{array}$ \\
\hline & \multicolumn{10}{|c|}{ Recreation } & \multicolumn{10}{|c|}{ Other } \\
\hline 16. SI & 16.46 & 16.84 & 17.12 & 17.03 & 16.86 & 14.98 & 15.16 & 15.16 & 14.81 & 15.03 & 31.32 & 31.77 & 31.80 & 79.53 & 43.61 & 41.70 & 49.81 & 49.93 & 102.14 & 60.90 \\
\hline 17. PI & 17.07 & 16.09 & 16.88 & 17.04 & 16.77 & 8.96 & 8.64 & 8.38 & 8.88 & 8.72 & 29.86 & 28.80 & 29.86 & 77.64 & 41.54 & 16.01 & 13.53 & 12.08 & 36.15 & 19.44 \\
\hline 18. $S M$ & 16.47 & 16.56 & 16.93 & 16.67 & 16.66 & 7.00 & 6.25 & 6.00 & 6.58 & 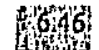 & 30.75 & 31.20 & 31.45 & 79.00 & 43.10 & 6.90 & 6.15 & 5.91 & 6.48 & 4636 \\
\hline 19. CEM & 16.10 & 16.21 & 16.59 & 14.34 & 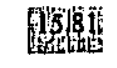 & 15.54 & 15.75 & 15.68 & 11.53 & 14.63 & 38.28 & 38.53 & 38.76 & 18.86 & 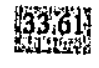 & 67.57 & 67.53 & 66.92 & 23.09 & 56.28 \\
\hline \multirow[t]{2}{*}{ 20. Menn } & 16.53 & 16.43 & 16.88 & 16.27 & 16.53 & 11.62 & 11.45 & 11.31 & 10.45 & 11.21 & 32.55 & 32.58 & 32.97 & 63.76 & 40.46 & 33.05 & 34.26 & 33.71 & 41.97 & 35.74 \\
\hline & \multicolumn{10}{|c|}{ Mean-Unweighted } & \multicolumn{10}{|c|}{ Mean-Weighted } \\
\hline 21.SI & 18.84 & 19.15 & 19.43 & 26.33 & 20.94 & 18.58 & 20.49 & 21.13 & 26.87 & 21.77 & 14.98 & 15.21 & 15.41 & 20.31 & 16.48 & 15.37 & 17.07 & 17.47 & 21.88 & 17.95 \\
\hline 22. PI & 18.49 & 17.64 & 18.52 & 24.95 & migor & 9.81 & 8.78 & 8.19 & 12.05 & 9.71 & 15.13 & 14.11 & 14.75 & 19.36 & Tras & 7.96 & 7.04 & 6.66 & 9.53 & 7.80 \\
\hline 23. SM & 18.68 & 18.92 & 19.29 & 26.14 & 20.76 & 6.60 & 5.89 & 5.65 & 6.20 & 管o & 14,86 & 15.03 & 15.31 & 20.19 & 16.35 & 6.07 & 5.41 & 5.19 & 5.69 & 55.59 \\
\hline 24. CEM & 21.25 & 21.48 & 21.86 & 16.18 & 20.19 & 24.91 & 24.57 & 24.45 & 13.81 & 21.94 & 16.49 & 16.63 & 16.91 & 13.37 & 15.85 & 20.15 & 19.65 & 19.44 & 11.77 & 17.75 \\
\hline 25. Menn & 19.32 & 19.30 & 19.78 & 23.40 & 20.45 & 14.98 & 14.93 & 14.86 & 14.73 & 14.87 & 15.37 & 15.25 & 15.60 & 18.31 & 16.13 & 12.39 & 12.29 & 12.19 & 12.22 & 12.27 \\
\hline
\end{tabular}

See notes to Table 11. 
Let this loss function take the general form $f(B, S)$, where $B$ is the bias and $S$ the standard error. It seems reasonable to postulate $f_{i} \geq 0$ for $i=B, S$ (where $\left.f_{i}=\partial f / \partial i\right)$, and $f_{i i} \geq 0$ (where $f_{i i}=\partial^{2} f / \partial i^{2}$ ). A simple functional form satisfying these properties is

$$
f(B, S)=\sqrt{\alpha B^{2}+(1-\alpha) S^{2}}
$$

where $0 \leq \alpha \leq 1$. According to (8.1), the square of the loss is a weighted average of the squared bias and the variance. It has the attraction of involving only one unknown parameter, $\alpha$. This function implies the following losses for various values of the parameter $\alpha$ :

$$
\frac{\alpha}{1}
$$$$
1 / 2
$$

0

\section{$\underline{\text { Loss }}$}

B

$(1 / \sqrt{2})$ RMSE

$S$

Accordingly, under (8.1) the numerical results in Tables 11,13 and 14 can be interpreted as values of the loss function when $\alpha$ is $1,1 / 2$, and 0 , respectively. ${ }^{11}$ Values of loss function (8.1) when $\alpha$ is .25 and .75 are given in the Appendix.

The relationship between the value of the loss function (8.1) and the parameter $\alpha$ for the four estimated models is shown in Figure 1. This figure is constructed from the means over the four alternative true models. As can be seen, when $\alpha$ increases

\footnotetext{
1 Strictly speaking, the RMSEs presented in Table 13 are proportional to the values of the loss function when $\alpha=1 / 2$.
} 
FIGURE 1

LOSSES FOR INCOME AND OWN-PRICE ELASTICITIES

Income elasticity

Own-price elasticity

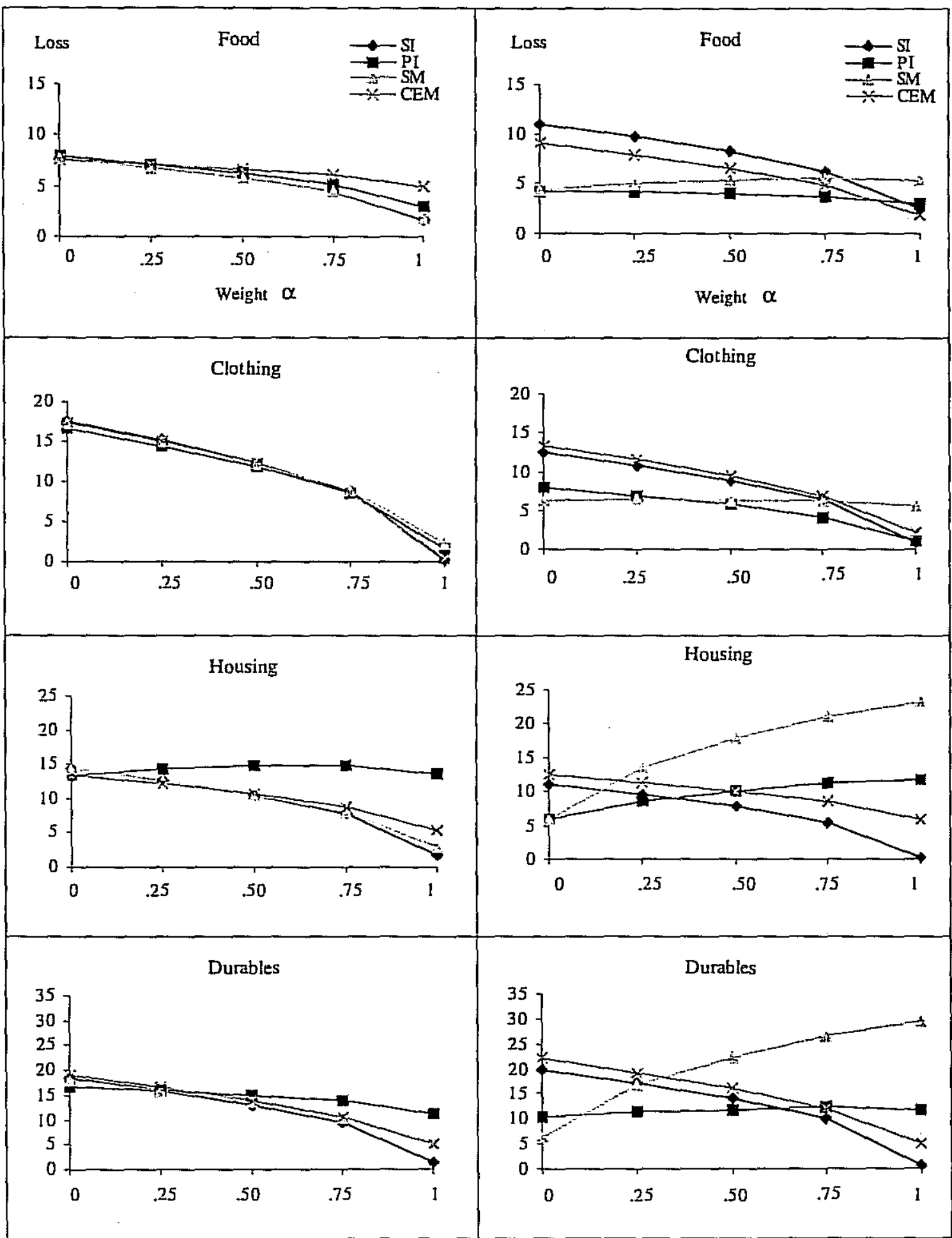

(Continued on next page) 
FIGURE 1 (continued)

LOSSES FOR INCOME AND OWN-PRICE ELASTICITIES

Income elasticity

Own-price elasticity

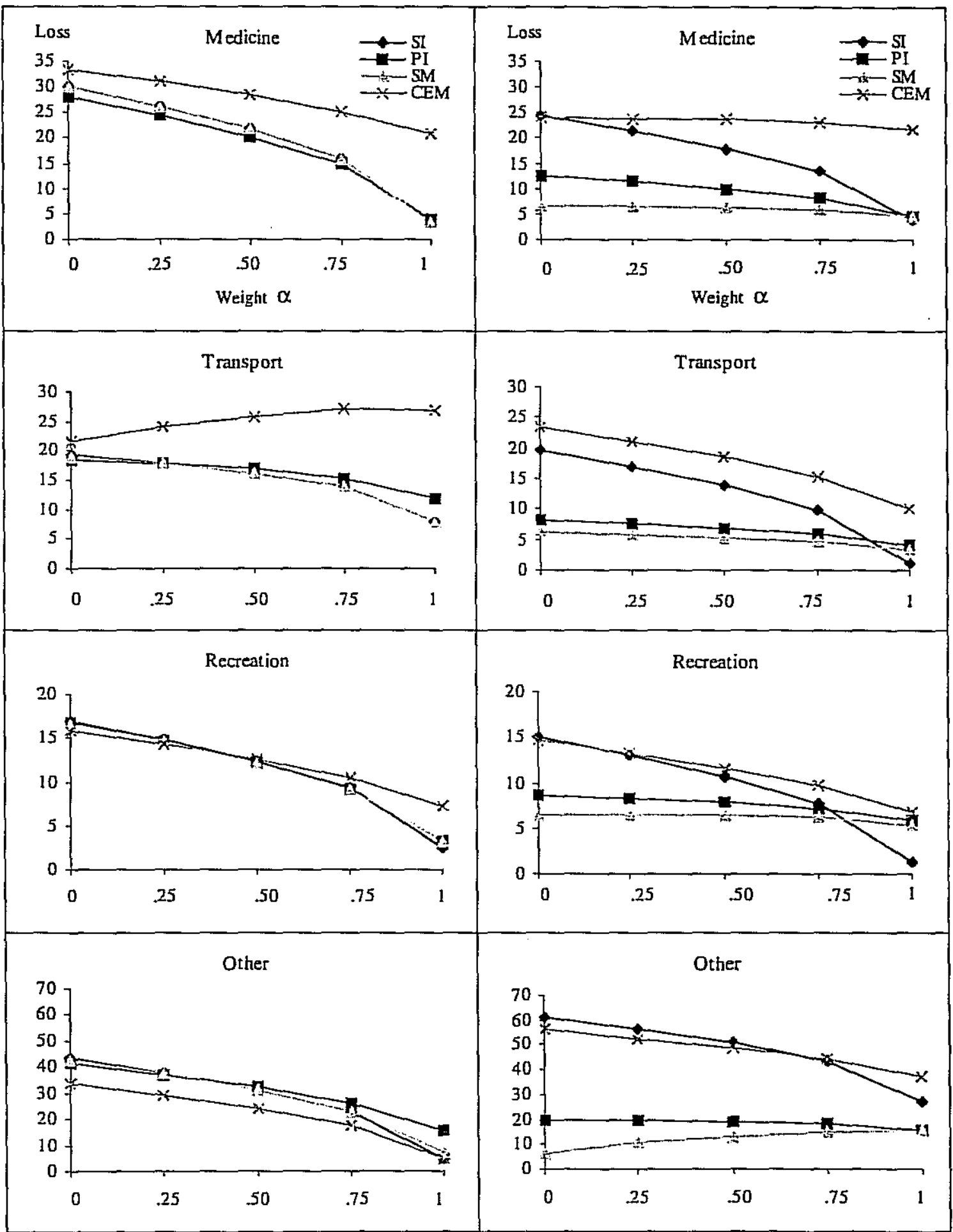

(Continued on next page) 
FIGURE 1 (continued)

LOSSES FOR INCOME AND OWN-PRICE ELASTICITIES

Income elasticity

Own-price elasticity

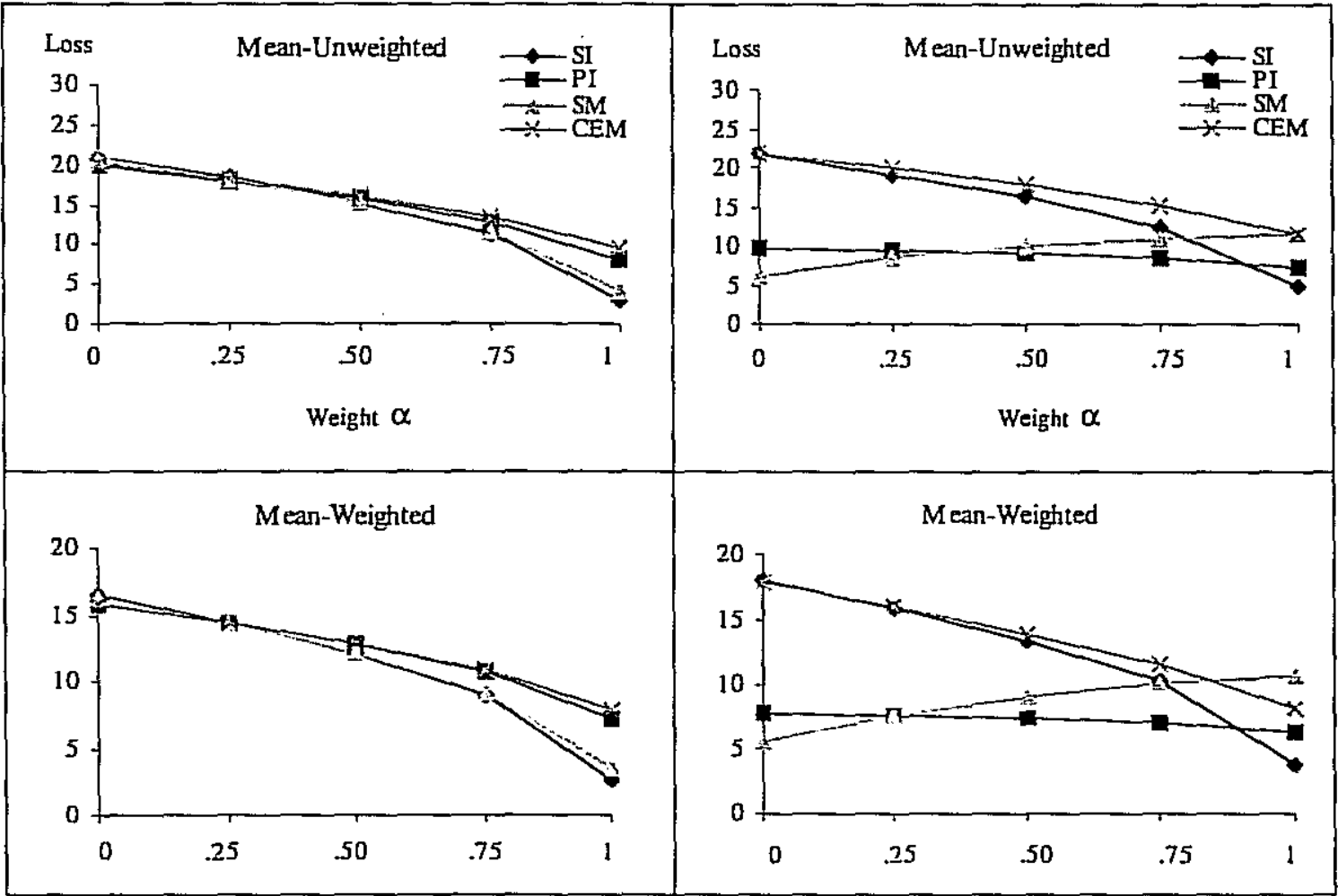

Note: All losses are to be divided by 100 .

from zero to one, the losses of the models generally decrease, indicating that on average the standard error is generally larger than the corresponding bias. Furthermore, for a given value of $\alpha$ and a given commodity group, the differences among the four models tend to be smaller for the income elasticity than the price elasticity. This agrees with common understanding that estimates of income elasticities tend to be more robust than those of price elasticities. To elaborate this further, write the loss as a function of the weight $\alpha$ and the estimated model (M), $\mathrm{g}(\alpha, \mathrm{M}), \quad$ where $\quad \alpha \in A, \quad A=[0, .25, .5, .75,1]$, and $\mathrm{M} \in M$, $M=$ [SI, PI, SM, CEM] . Then for a given value of $\alpha$, one measure of dispersion across models is the standard deviation 


$$
\sigma_{M}(\alpha)=\sqrt{\frac{1}{4} \sum_{M \in M}[\mathrm{~g}(\alpha, M)-\mathrm{g}(\alpha, \cdot)]^{2}}
$$

where $g(\alpha, \cdot)=(1 / 4) \sum_{M \in M} g(\alpha, M)$ is the mean over the four models of the loss for a given value of $\alpha$. Columns 2 and 3 of Table 15 present (8.2), averaged over the five values of $\alpha$ for each of the ten groups. As can be seen, the dispersion among the losses for the own-price elasticities is much greater than that for the income elasticities; on a weighted basis, the dispersion of the price elasticities is almost five times that of the income elasticities. This agrees with common understanding that estimates of income elasticities tend to be more robust than those of price elasticities. The analogous standard deviation across the five values of $\alpha$ for model $M$ is:

$$
\sigma_{\alpha}(M)=\sqrt{\frac{1}{5} \sum_{\alpha \in A}[g(\alpha, M)-g(\cdot, M)]^{2}},
$$

where $g(\cdot, M)=(1 / 5) \sum_{\alpha \in A} g(\alpha, M)$ is the mean over the five values of $\alpha$ for model $M$. Columns 4 and 5 of Table 15 present (8.3) averaged over the four models for each of the ten groups. In contrast to before, there is now a tendency for the ownprice elasticities to have a lower dispersion than the income elasticities.

As Figure 1 reveals that, in general, the losses for the income elasticities are fairly similar for all models, ${ }^{12}$ there is little to choose between the four models on this basis. This could be a reflection of Working's model underlying the income response in three of the four cases. The situation regarding the losses with respect to the price elasticities is more revealing, however. Note that for the price elasticities of Housing and Durables, Selvanathan's model does not perform well with respect to the bias (i.e., for high values of the weight $\alpha$ ). However, this is more or less offset by the superior performance of this model with respect to (i) the other commodities and (ii) the variability of the elasticity estimates (low values of $\alpha$ ). As a result, on average Selvanathan's model performs best with respect to the price elasticities.

\footnotetext{
${ }^{12}$ Housing, Transport and, possibly, Medicine are the exceptions to this general rule.
} 
TABLE 15

STANDARD DEVIATIONS OF THE LOSSES

\begin{tabular}{lcccc}
\hline & \multicolumn{2}{c}{ Over estimated models } & \multicolumn{2}{c}{ Over the weight $\alpha$} \\
\cline { 2 - 5 } Commodity & $\begin{array}{c}\text { Income } \\
\text { elasticity }\end{array}$ & $\begin{array}{c}\text { Own-price } \\
\text { elasticity }\end{array}$ & $\begin{array}{c}\text { Income } \\
\text { elasticity }\end{array}$ & $\begin{array}{c}\text { Own-price } \\
\text { elasticity } \\
\text { (1) }\end{array}$ \\
\hline Food & $(2)$ & $(3)$ & $(4)$ & $(5)$ \\
Clothing & .53 & 1.80 & 1.69 & 1.59 \\
Housing & .35 & 1.97 & 5.59 & 2.66 \\
Durables & 2.14 & 4.59 & 2.96 & 3.56 \\
Medicine & 1.44 & 6.15 & 4.27 & 5.34 \\
Transport & 3.82 & 7.15 & 7.91 & 2.88 \\
Recreation & 4.25 & 5.29 & 3.07 & 3.38 \\
Other & .63 & 2.44 & 4.38 & 2.25 \\
Mean-Unweighted & 3.73 & 16.63 & 11.32 & 5.78 \\
Mean-Weighted & .90 & 4.34 & 5.00 & 3.12 \\
\hline
\end{tabular}

Note: All entries are to be divided by 100 .

The performance of the models for alternative values of $\alpha$ is summarised in Table 16, which is an extension of Table 12. For a given value of $\alpha$, this table gives the frequency that a model is ranked the best with respect to the value of the loss function. As can be seen from column 2 in the top panel referring the income elasticities, when $\alpha=1$ SI has the lowest loss 88 percent of the time. As this is the highest value in the column, we can say that this model performs best for $\alpha=1$, whereby the loss coincides with the bias. Similarly, from column 6, PI is the best for $\alpha=0$ (the standard error). It is to be noted that SI also dominates in columns 3 and 4 ( $\alpha=.75, .5$ ). Averaging over the five values of $\alpha$ yields the means given in column 7, which shows that, on average, SI performs the best 38 percent of the time. Then follows PI (30 percent), CEM (22 percent), while SM is the worst performer (10 percent). The middle panel of the table refers to the own-price elasticities. It shows that SI dominates only when $\alpha=1$, while SM wins for the other four values of $\alpha$, as 
TABLE 16

SECOND SUMMARY OF PERFORMANCE OF MODELS

(Percentage of cases when model is ranked first)

\begin{tabular}{|c|c|c|c|c|c|c|}
\hline \multirow{3}{*}{ Model } & \multicolumn{5}{|c|}{ Value of loss function parameter } & \multirow{3}{*}{ Mean } \\
\hline & $\alpha=1$ & $\alpha=.75$ & $\alpha=.5$ & $\alpha=.25$ & $\alpha=0$ & \\
\hline & BIAS & & $(1 / \sqrt{2}) \mathrm{RMS}$ & & $\mathrm{SE}$ & \\
\hline \multirow[t]{2}{*}{ (1) } & (2) & (3) & (4) & $(5)$ & (6) & (7) \\
\hline & \multicolumn{6}{|c|}{ Income elasticities } \\
\hline 1. SI & 895 & 政000 & 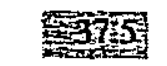 & 12.5 & 0.0 & 蕰 \\
\hline 2. PI & 0.0 & 37.5 & 25.0 & 25.0 & 625 & 30.0 \\
\hline 3. SM & 0.0 & 0.0 & 25.0 & 25.0 & 0.0 & 10.0 \\
\hline 4. CEM & 12.5 & 12.5 & 12.5 & 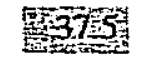 & 37.5 & 22.5 \\
\hline \multirow[t]{2}{*}{ 5.Total } & 100.0 & 100.0 & 100.0 & 100.0 & 100.0 & 100.0 \\
\hline & \multicolumn{6}{|c|}{ Own-price elasticities } \\
\hline 6. SI & 750 & 25.0 & 12.5 & 0.0 & 0.0 & 22.5 \\
\hline 7. PI & 0.0 & 25.0 & 37.5 & 37.5 & 12.5 & 22.5 \\
\hline 8. SM & 12.5 & 9500 & 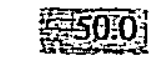 & 625 & 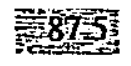 & 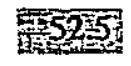 \\
\hline 9. CEM & 12.5 & 0.0 & 0.0 & 0.0 & 0.0 & 2.5 \\
\hline \multirow[t]{2}{*}{ 10. Total } & 100.0 & 100.0 & 100.0 & 100.0 & 100.0 & 100.0 \\
\hline & \multicolumn{6}{|c|}{ Income and own-price elasticities } \\
\hline 11. SI & 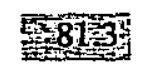 & 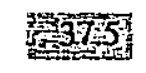 & 25.0 & 6.3 & 0.0 & 30.0 \\
\hline 12. PI & 0.0 & 31.3 & 31.3 & 31.2 & 37.5 & 26.3 \\
\hline 13. SM & 6.2 & 25.0 & 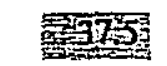 & 438 & is & 20 \\
\hline 14. CEM & 12.5 & 6.2 & 6.2 & 18.7 & 18.7 & 12.5 \\
\hline 15. Total & 100.0 & 100.0 & 100.0 & 100.0 & 100.0 & 100.0 \\
\hline
\end{tabular}

See notes to Table 12.

well for the mean. The performance of the models on the basis of the income and price elasticities combined is given in the bottom panel of Table 16. As can be seen, SI dominates for $\alpha=1$ and .75 , while SM wins for $\alpha=.5, .25$ and 0 . On 
average for both types of elasticities, SM appears the best. Figure 2 plots the information in the bottom panel of this table.

FIGURE 2

MODEL PERFORMANCE

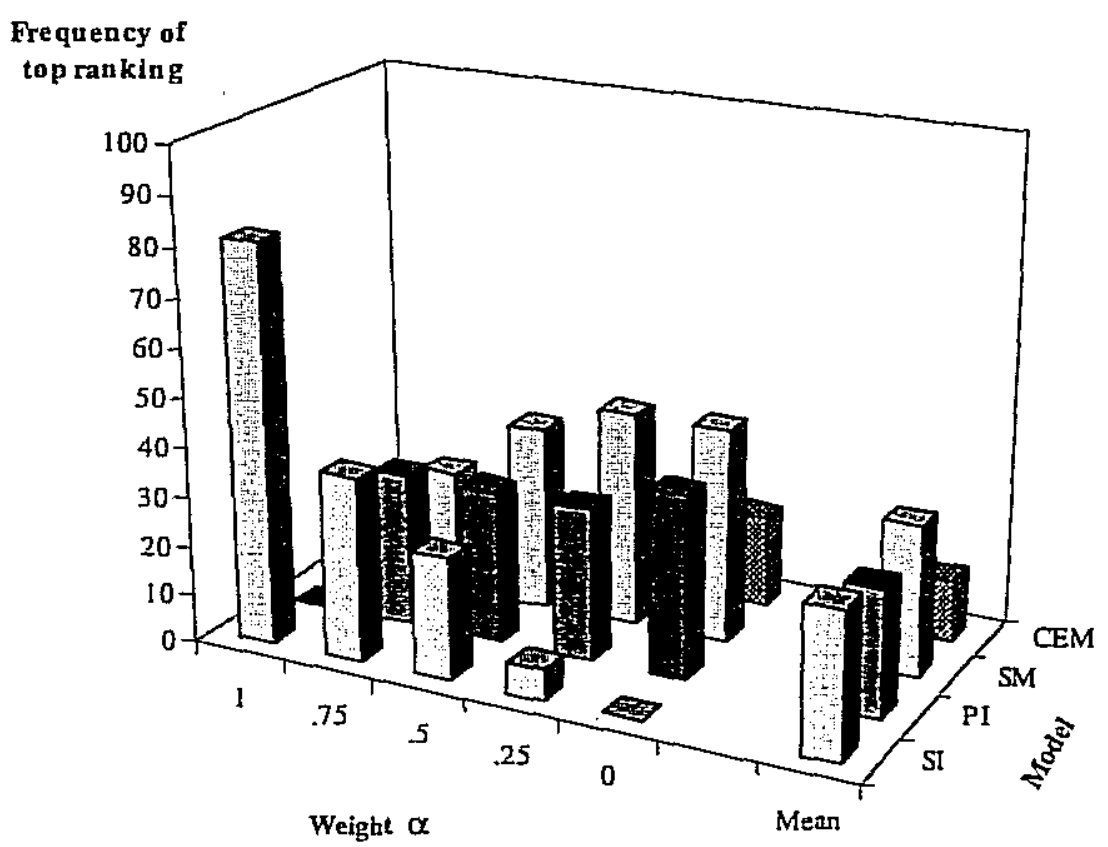

While Table 16 represents a summary picture of the results for all commodity groups, Table 17 presents some more detail by giving the rankings of the models, according to the values of the loss function (8.1), for food, which, as it has the largest budget share in most countries, it is the most prominent single commodity. As can be seen from rows 1-4 of the table, SI is the top-ranked model with respect to the food income elasticity for four out of the five values of the loss function parameter $\alpha$. The picture is a little different, however, regarding the price elasticity as shown in rows 58. As the parameter $\alpha$ declines from 1 to .75 , the top-ranked model moves from CEM to PI model and stays there for the other three values of $\alpha$. On the basis of the mode (column 7), Working's model under preference independence dominates for the 
price elasticity of food. The bottom panel of Table 17 is constructed by averaging the losses for the income and the own-price elasticities of food and then ranking the models accordingly. Now SI dominates for $\alpha=1$, where the loss is the bias, while SM wins for $\alpha=0$, where the loss is the standard error. In other words, SI is the best choice for food when the bias of the elasticities is of major concern, while SM is preferred when the standard error is of great importance. On the basis of the mode (column 7), on average PI dominates here.

TABLE 17

RANKINGS OF MODELS FOR FOOD

\begin{tabular}{|c|c|c|c|c|c|c|}
\hline \multirow{3}{*}{ Model } & \multicolumn{5}{|c|}{ Value of loss function parameter } & \multirow{3}{*}{ Mode } \\
\hline & $\alpha=1$ & $\alpha=.75$ & $\alpha=.5$ & $\alpha=.25$ & $\alpha=0$ & \\
\hline & BIAS & \multicolumn{3}{|c|}{$(1 / \sqrt{2})$ RMSE } & SE & \\
\hline (1) & (2) & (3) & (4) & (5) & (6) & (7) \\
\hline \multicolumn{7}{|c|}{ Income elasticity } \\
\hline 1. SI & 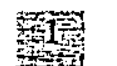 & 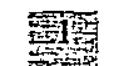 & 望语 & 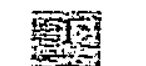 & 3 & 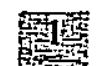 \\
\hline 2. PI & 3 & 3 & 3 & 3 & 4 & 3 \\
\hline 3. SM & 2 & 2 & 2 & 2 & 2 & 2 \\
\hline 4. CEM & 4 & 4 & 4 & 4 & 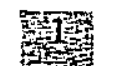 & 4 \\
\hline \multicolumn{7}{|c|}{ Own-price elasticity } \\
\hline 5. SI & 2 & 4 & 4 & 4 & 4 & 4 \\
\hline 6. PI & 3 & 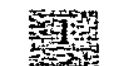 & 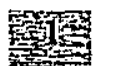 & 留㧼 & 㼭垔 & 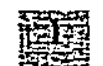 \\
\hline 7. SM & 4 & 3 & 2 & 2 & 2 & 2 \\
\hline 8. CEM & 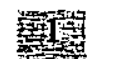 & 2 & 3 & 3 & 3 & 3 \\
\hline \multicolumn{7}{|c|}{ Income and own-price elasticities } \\
\hline 9. SI & 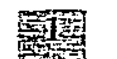 & 3 & 4 & 4 & 4 & 4 \\
\hline 10. PI & 2 & 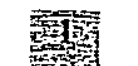 & 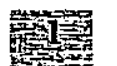 & 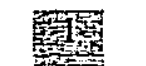 & 2 & 17 \\
\hline 11. SM & 3 & 2 & 2 & 2 & 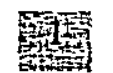 & 2 \\
\hline 12. CEM & 4 & 4 & 3 & 3 & 3 & 3 \\
\hline
\end{tabular}

Notes: 1 . SI $=$ Working's model under substitution independence, PI $=$ Working's model under preference independence, $S M=$ Selvanathan's model, and CEM = Constant elasticity model.

2. Shaded cells indicate the top-ranked models. 


\section{CONCLUSIONS}

An important practical problem in economics is how to estimate demand elasticities. Given the availability of a large number of functional forms of demand equations, each of which would most likely yield different numerical values of elasticities, the question of which model is "best" would seem to be difficult to resolve. This difficulty is compounded by the claims and counterclaims made by advocates of particular models.

The concept of "flexibility" of demand models has received some prominence in the literature (Diewert, 1971). A demand model is said to be flexible if it has a sufficient number of parameters to represent a second-order approximation to the underlying generating function (the utility function, direct or indirect, expenditure function, etc.). But as flexible functional forms necessarily contain a large number of unknown parameters, in practice they are not as useful as would seem to be the case, especially when the number of goods (n) is moderate to large as the number of parameters is of the order of $\mathrm{n}^{2}$. Another approach is to choose between functional forms on the basis of some goodness-of-fit criterion such as the information inaccuracy or the value of the likelihood function. While this is a popular approach, it still does not seem to have revealed any strong general findings that, for example, a particular model tends to dominate time and time again -- the results seem to be specific to the application in question involving specific data and particular estimation procedures.

In this paper, we have proposed a new approach to the problem. A matrix approach to simulating (MAS) demand equations is developed to analyse the performance of demand models under idealised circumstances. Artificial data on the dependent variable are generated by one model, which is referred to as the "true" model. These data are then used for the estimation of another model, referred to as the "estimated" model. If $\mathrm{i}$ denotes the estimated model and $\mathrm{j}$ the true model, then MAS considers all pairs $(i, j)$ for $i, j=1, \ldots, m$, where $m$ is the number of models 
investigated. This yields an $\mathrm{m} \times \mathrm{m}$ comparison matrix, which gives all pair-wise comparisons of the alternative models. As the primary focus of demand analysis is usually on the values of income and price elasticities, we use the quality of the estimated elasticities as the criterion for evaluating the performance of the various models. We employ a loss function the square of which is a weighted average (with weight parameter $\alpha$ ) of the squared bias and the variance of each estimated elasticity. The attraction of this loss function is that it coincides with the bias and the standard error of the elasticity when $\alpha=1$ and 0 , respectively, and is proportional to the RMSE when $\alpha=1 / 2$. The $m$ estimated models are then compared on the basis of this loss function, averaged across the $\mathrm{m}$ alternative true models. Since values of the loss function are dependent on the weight parameter $\alpha$, the rankings of models may vary with different values of $\alpha$. Accordingly, the overall result is obtained by averaging the loss function over $\alpha$.

We use cross-country data involving 42 countries and 8 commodities to compare four models, namely Working's model under substitution independence (SD), Working's model under preference independence (PI), Selvanathan's model (SM) and the constant elasticity model (CEM). Whilst these are all simple models, they are commonly used in practice, especially when the quality and/or the variability of the data are modest. Employing the MAS methodology yields a $4 \times 4$ matrix of pairwise comparisons of the models, for each of the eight commodities and two elasticities, viz., the income and own-price elasticities. In general, the four models perform similarly with respect to the income elasticity estimates, while there is considerably more difference regarding the price elasticities. The results, on the basis of the eight commodities combined, are summarised in the top panel of Table 18 . This shows that:

- On average (over the various values of the weight parameter $\alpha$ in the loss function), the SI model performs best regarding the income elasticities, while SM wins for the price elasticities. On the basis of the income and price elasticities combined, SM again ranks number one. 
- When the bias of the elasticities is of major concern, whereby the weight parameter $\alpha=1$, SI dominates for the income and price elasticities, both by themselves and combined.

- When the RMSEs are given prominence $(\alpha=1 / 2)$, SI dominates only for the income elasticities, while SM wins for the price elasticities and the income and price elasticities combined.

- When the standard errors are of great importance $(\alpha=0)$, PI dominates for the income elasticities, while SM performs best for both the price elasticities and the income and price elasticities combined.

TABLE 18

SUMMARY OF RESULTS

\begin{tabular}{|c|c|c|c|c|}
\hline \multirow{2}{*}{$\begin{array}{l}\text { Value of } \\
\text { weight } \alpha\end{array}$} & \multirow{2}{*}{$\begin{array}{l}\text { Interpretation of } \\
\text { loss function }\end{array}$} & \multicolumn{3}{|c|}{ Preferred model on the basis of } \\
\hline & & $\begin{array}{l}\text { Income } \\
\text { elasticities }\end{array}$ & $\begin{array}{l}\text { Own-price } \\
\text { elasticities }\end{array}$ & $\begin{array}{l}\text { Income and own- } \\
\text { price elasticities }\end{array}$ \\
\hline & \multicolumn{4}{|c|}{ Eight commodities combined } \\
\hline Average & Central tendency & SI & SM & SM \\
\hline 1 & Bias & SI & SI & SI \\
\hline $1 / 2$ & RMSE & SI & SM & SM \\
\hline \multirow[t]{2}{*}{0} & Standard error & PI & SM & $S M$ \\
\hline & \multicolumn{4}{|c|}{ Food } \\
\hline Average & Central tendency & SI & PI & PI \\
\hline 1 & Bias & SI & CEM & SI \\
\hline $1 / 2$ & RMSE & SI & PI & PI \\
\hline 0 & Standard error & CEM & PI & SM \\
\hline
\end{tabular}

Note: $\mathrm{SI}=$ Working's model under substitution independence, $\mathrm{PI}=$ Working's model under preference independence, $\mathrm{SM}=$ Selvanathan's model, and CEM = Constant elasticity model. 
As food is the most prominent single commodity, the values of its demand elasticities are of particular interest. The results for food are summarised in the lower panel of Table 18. Overall, PI is the preferred model if the analyst is particularly interested in food demand elasticities. 


\section{APPENDLX}

This appendix gives some further values of the loss function (8.1). These are presented in Tables A1 and A2 for when the weight $\alpha$ is set at .25 and .75. 
TABLE A1

LOSSES FOR INCOME AND OWN-PRICE ELASTICITIES WHEN $\alpha=.25$

\begin{tabular}{|c|c|c|c|c|c|c|c|c|c|c|c|c|c|c|c|c|c|c|c|c|}
\hline \multirow{2}{*}{$\begin{array}{l}\text { True } \\
\text { model } \\
\text { Estimnted } \\
\text { model }\end{array}$} & \multicolumn{5}{|c|}{ Income elasticity } & \multicolumn{5}{|c|}{ Own-price elasticity } & \multicolumn{5}{|c|}{ Income elasticity } & \multicolumn{5}{|c|}{ Own-price elasticity } \\
\hline & $\begin{array}{l}\text { SI } \\
\text { (1) }\end{array}$ & $\begin{array}{l}\text { PI } \\
\text { (2) }\end{array}$ & $\begin{array}{l}S M \\
\text { (3) }\end{array}$ & $\begin{array}{c}\text { CEM } \\
\text { (4) }\end{array}$ & $\begin{array}{c}\text { Mean } \\
\text { (5) }\end{array}$ & $\begin{array}{l}\text { SI } \\
(6)\end{array}$ & $\begin{array}{l}\text { PI } \\
(7)\end{array}$ & $\begin{array}{l}S M \\
(8)\end{array}$ & $\begin{array}{c}\text { CEM } \\
(9)\end{array}$ & $\begin{array}{c}\text { Mean } \\
(10)\end{array}$ & $\begin{array}{c}\text { SI } \\
(11)\end{array}$ & $\begin{array}{l}\text { PI } \\
\text { (12) }\end{array}$ & $\begin{array}{l}S M \\
(13)\end{array}$ & $\begin{array}{l}\text { CEM } \\
(14)\end{array}$ & $\begin{array}{c}\text { Menn } \\
(15)\end{array}$ & $\begin{array}{c}\text { SI } \\
(16)\end{array}$ & $\begin{array}{c}\text { PI } \\
(17)\end{array}$ & $\begin{array}{l}S M \\
(18)\end{array}$ & $\begin{array}{l}\text { CEM } \\
\text { (19) }\end{array}$ & $\begin{array}{l}\text { Mean } \\
(20)\end{array}$ \\
\hline & \multicolumn{10}{|c|}{ Food } & \multicolumn{10}{|c|}{ Clothing } \\
\hline 1. SI & 6.89 & 6.72 & 6.50 & 6.92 & 数6 & 8.59 & 9.68 & 9.40 & 11.17 & 9.71 & 14.16 & 14.33 & 14.53 & 17.41 & 15.11 & 10.53 & 10.90 & 11.03 & 10.90 & 10.84 \\
\hline 2. PI & 8.30 & 6.58 & 6.55 & 6.94 & 7.09 & $4.2 \mathrm{~B}$ & 3.42 & 4.64 & 4.39 & Pigi & 13.95 & 13.69 & 14.26 & 15.86 & 14 & 7.45 & 6.91 & 6.27 & 7.02 & 6.91 \\
\hline 3. SM & 6.90 & 6.66 & 6.55 & 6.95 & 6.76 & 4.55 & 4.69 & 3.57 & 7.13 & 4.99 & 14.15 & 14.16 & 14.27 & 17.44 & 15.01 & 8.20 & 6.07 & 5.07 & 6.05 & 地 \\
\hline 4. CEM & 7.74 & 7.59 & 7.48 & 5.73 & 7.14 & 9.35 & 8.42 & 7.93 & 6.29 & 8.00 & 14.78 & 14.81 & 14.92 & 15.21 & 14.93 & 12.54 & 11.96 & 11.53 & 10.18 & 11.55 \\
\hline \multirow[t]{2}{*}{ 5. Mean } & 7.46 & 6.89 & 6.77 & 6.63 & 6.94 & 6.69 & 6.55 & 6.38 & 7.24 & 6.72 & 14.26 & 14.25 & 14.50 & 16.48 & 14.87 & 9.68 & 8.96 & 8.47 & 8.54 & 8.91 \\
\hline & \multicolumn{10}{|c|}{ Housing } & \multicolumn{10}{|c|}{ Durables } \\
\hline 6. SI & 10.98 & 12.09 & 12.96 & 14.37 & 12.60 & 8.48 & 9.55 & 10.08 & 9.84 & 9.49 & 14.98 & 15.28 & 16.38 & 16.93 & 15.89 & 16.00 & 17.32 & 19.17 & 16.47 & 17.24 \\
\hline 7. PI & 20.36 & 10.55 & 12.43 & 14.00 & 14.33 & 16.14 & 4.74 & 7.00 & 6.76 & 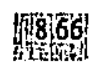 & 18.65 & 13.67 & 15.85 & 15.62 & 15.95 & 13.70 & 8.63 & 10.68 & 12.30 & 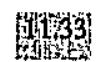 \\
\hline 8. SM & 10.98 & 12.01 & 12.90 & 14.59 & 12.62 & 26.95 & 10.82 & 4.76 & 11,73 & 13.56 & 15.07 & 15.08 & 16.06 & 17.23 & M 186 & 27.09 & 14.54 & 5.16 & 19.91 & 16.68 \\
\hline 9. CEM & 12.12 & 12.29 & 12.69 & 11.48 & 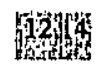 & 12.59 & 13.36 & 14.24 & 5.29 & 11.37 & 16.56 & 17.08 & 18.19 & 14.78 & $\frac{16.65}{16.65}$ & 21.87 & 20.94 & 21.55 & 12.90 & 19.31 \\
\hline \multirow[t]{2}{*}{ 10. Mean } & 13.61 & 11.74 & 12.74 & 13.61 & 12.92 & 16.04 & 9.62 & 9.02 & 8.40 & 10.77 & 16.31 & 15.28 & 16.62 & 16.14 & 16.09 & 19.67 & 15.36 & 14.14 & 15.40 & 16.14 \\
\hline & \multicolumn{10}{|c|}{ Medicine } & \multicolumn{10}{|c|}{ Transport } \\
\hline $11, \mathrm{SI}$ & 26.60 & 26.69 & 26.18 & 25.42 & 26.22 & 20.82 & 21.82 & 22.50 & 20.33 & 21.37 & 15.56 & 15,47 & 15.68 & 24.75 & 17.87 & 15.23 & 16.44 & 17.89 & 18.08 & 16.91 \\
\hline 12. $\mathrm{PI}$ & 24.97 & 24.27 & 25.06 & 23.40 & 20142 & 12.49 & 11.09 & 9.96 & 12.10 & 11.41 & 15.69 & 14.57 & 15.21 & 26.34 & 17.95 & 9.07 & 6.84 & 6.33 & 7.92 & 7.54 \\
\hline 13. SM & 26.67 & 26.64 & 26.47 & 25.11 & 26.22 & 6.26 & 5.66 & 5.33 & 9.21 & 676,62 & 15.51 & 15.36 & 15.52 & 24.66 & Hitio & 5.82 & 6.19 & 4.97 & 6.15 & 5508 ? \\
\hline 14. CEM & 35.51 & 35.55 & 35.06 & 17.53 & 30.91 & 28.34 & 28.38 & 27.71 & 11.30 & 23.93 & 25.74 & 26.03 & 25.54 & 18.63 & 23.99 & 22.30 & 21.40 & 21.02 & 19.78 & 21.12 \\
\hline 15. Mean & 28.44 & 28.29 & 28.19 & 22.87 & 26.95 & 16.98 & 16.74 & 16.37 & 13.23 & 15.83 & 18.12 & 17.86 & 17.99 & 23.60 & 19.39 & 13.11 & 12.72 & 12.55 & 12.98 & 12.84 \\
\hline
\end{tabular}

(Continued on next page) 
TABLE A1 (continued)

LOSSES FOR INCOME AND OWN-PRICE ELASTICITIES WHEN $\alpha=.25$

\begin{tabular}{|c|c|c|c|c|c|c|c|c|c|c|c|c|c|c|c|c|c|c|c|c|}
\hline \multirow[b]{2}{*}{$\begin{array}{l}\text { Estimate } \\
\text { model }\end{array}$} & \multicolumn{5}{|c|}{ Income elasticity } & \multicolumn{5}{|c|}{ Own-price elasticity } & \multicolumn{5}{|c|}{ Income elasticity } & \multicolumn{5}{|c|}{ Own-price elasticity } \\
\hline & $\begin{array}{l}\text { SI } \\
(1)\end{array}$ & $\begin{array}{l}\text { PI } \\
\text { (2) }\end{array}$ & $\begin{array}{l}\text { SM } \\
\text { (3) }\end{array}$ & $\begin{array}{l}\text { CEM } \\
(4)\end{array}$ & $\begin{array}{l}\text { Mean } \\
\text { (5) }\end{array}$ & $\begin{array}{l}\text { SI } \\
(6)\end{array}$ & $\begin{array}{l}\text { PI } \\
\text { (7) }\end{array}$ & $\begin{array}{l}S M \\
(8)\end{array}$ & $\begin{array}{c}\text { CEM } \\
(9)\end{array}$ & $\begin{array}{c}\text { Mesn } \\
(10)\end{array}$ & $\begin{array}{c}\text { SI } \\
\text { (11) }\end{array}$ & $\begin{array}{c}\text { PI } \\
(12)\end{array}$ & $\begin{array}{l}S M \\
(13)\end{array}$ & $\begin{array}{l}\text { CEM } \\
\text { (14) }\end{array}$ & $\begin{array}{c}\text { Mean } \\
(15)\end{array}$ & $\begin{array}{c}\text { SI } \\
(16)\end{array}$ & $\begin{array}{c}\text { PI } \\
(17)\end{array}$ & $\begin{array}{l}S M \\
(18)\end{array}$ & $\begin{array}{l}\text { CEM } \\
\text { (19) }\end{array}$ & $\begin{array}{c}\text { Mean } \\
(20)\end{array}$ \\
\hline & \multicolumn{10}{|c|}{ Recreation } & \multicolumn{10}{|c|}{ Other } \\
\hline 16. SI & 14.25 & 14.58 & 14.83 & 15.51 & 14.79 & 12.98 & 13.14 & 13.14 & 12.89 & 13.04 & 27.12 & 27.51 & 27.54 & 69.43 & 37.90 & 36.11 & 43.14 & 43.25 & 102.88 & 56.35 \\
\hline 17. PI & 15.11 & 13.93 & 14.63 & 14.98 & 14.67 & 8.12 & 7.48 & 7.82 & 9.90 & 8.33 & 27.18 & 24.94 & 25.86 & 70.79 & 37.20 & 22.33 & 11.72 & 10.54 & 33.74 & 19.58 \\
\hline 18. SM & 14.29 & 14.37 & 14.66 & 15.10 & 14.61 & 7.71 & 7.31 & 5.20 & 5.76 & 46 & 26.76 & 27.02 & 27.24 & 69.40 & 37.60 & 25.68 & 6.61 & 5.12 & 6.10 & 10,88 \\
\hline 19. CEM & 14.73 & 14.78 & 15.09 & 12.42 & 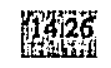 & 13.96 & 14.47 & 14.46 & 9.99 & 13.22 & 33.36 & 33.50 & 33.65 & 16.33 & 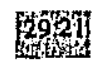 & 65.78 & 62.57 & 62.19 & 20,00 & 52.64 \\
\hline \multirow[t]{2}{*}{ 20. Mean } & 14.60 & 14.42 & 14.80 & 14.50 & 14.58 & 10.69 & 10.60 & 10.15 & 9.64 & 10.27 & 28.61 & 28.25 & 28.57 & 56.49 & 35.48 & 37.48 & 31.01 & 30.27 & 40.68 & 34.86 \\
\hline & \multicolumn{10}{|c|}{ Mean-Unweighted } & \multicolumn{10}{|c|}{ Mean-Weighted } \\
\hline 21. SI & 16.32 & 16.58 & 16.83 & 23.44 & 18.29 & 16.09 & 17,75 & 18.30 & 24.78 & 19.23 & 12.97 & 13.17 & 13.35 & 18.24 & 14.43 & 13.31 & 14.79 & 15.13 & 20.07 & 15.83 \\
\hline 22. PI & 17.10 & 15.28 & 16.14 & 23.06 & 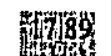 & 10.85 & 7.60 & 7.52 & 11.64 & 9.40 & 14.35 & 12.22 & 12.88 & 18.12 & 14.39 & 8.93 & 6.10 & 6.42 & 9.17 & 7.65 \\
\hline 23. SM & 16.24 & 16.40 & 16.71 & 23.48 & 18.21 & 12.76 & 7.35 & 4.89 & 8.35 & $48 \times 4$ & 12.91 & 13.03 & 13.26 & 18.32 & 1 & 11.08 & 6.79 & 4.50 & 8.05 & (760] \\
\hline 24. CEM & 19.54 & 19.64 & 19.77 & 14.01 & 18.24 & 23.05 & 22.51 & 22.37 & 11.96 & 19.97 & 15.33 & 15.36 & 15.40 & 11.58 & 14.42 & 18.37 & 17,77 & 17.57 & 10.20 & 15.97 \\
\hline 25. Menn & 17.30 & 16.98 & 17.36 & 21.00 & 18.16 & 15.69 & 13.80 & 13.27 & 14.18 & 14.24 & 13.89 & 13.44 & 13.72 & 16.57 & 14.41 & 12.92 & 11.36 & 10.90 & 11.87 & 11.76 \\
\hline
\end{tabular}

See notes to Table 11. 
TABLE A2

LOSSES FOR INCOME AND OWN-PRICE ELASTICITIES WHEN $\alpha=.75$

\begin{tabular}{|c|c|c|c|c|c|c|c|c|c|c|c|c|c|c|c|c|c|c|c|c|}
\hline \multirow[b]{2}{*}{$\begin{array}{l}\text { Estimated } \\
\text { model }\end{array}$} & \multicolumn{5}{|c|}{ Income elasticity } & \multicolumn{5}{|c|}{ Own-price elasticity } & \multicolumn{5}{|c|}{ Income elasticity } & \multicolumn{5}{|c|}{ Own-price elasticity } \\
\hline & $\begin{array}{l}\text { SI } \\
\text { (I) }\end{array}$ & $\begin{array}{l}\text { PI } \\
(2)\end{array}$ & $\begin{array}{l}S M \\
(3)\end{array}$ & $\begin{array}{l}\text { CEM } \\
\text { (4) }\end{array}$ & $\begin{array}{c}\text { Mean } \\
(5)\end{array}$ & $\begin{array}{l}\text { SI } \\
(6)\end{array}$ & $\begin{array}{l}\text { PI } \\
\text { (7) }\end{array}$ & $\begin{array}{l}S M \\
(8)\end{array}$ & $\begin{array}{c}\text { CEM } \\
\text { (9) }\end{array}$ & $\begin{array}{l}\text { Mean } \\
(10)\end{array}$ & $\begin{array}{c}\text { SI } \\
(11)\end{array}$ & $\begin{array}{c}\text { PI } \\
\text { (12) }\end{array}$ & $\begin{array}{l}S M \\
(13)\end{array}$ & $\begin{array}{l}\text { CEM } \\
(14)\end{array}$ & $\begin{array}{c}\text { Mean } \\
(15)\end{array}$ & $\begin{array}{c}\text { SI } \\
(16)\end{array}$ & $\begin{array}{c}\text { PI } \\
\text { (17) }\end{array}$ & $\begin{array}{l}S M \\
(18)\end{array}$ & $\begin{array}{l}\text { CEM } \\
(19)\end{array}$ & $\begin{array}{l}\text { Mean } \\
(20)\end{array}$ \\
\hline & \multicolumn{10}{|c|}{ Food } & \multicolumn{10}{|c|}{ Clothing } \\
\hline 1. SI & 3.98 & 3.88 & 3.76 & 6.34 & 444 & 5.00 & 5.64 & 5.48 & 8.85 & 6.24 & 8.18 & 8.28 & 8.40 & 10.05 & 8.73 & 6.08 & 6.29 & 6.37 & 6.67 & 6.35 \\
\hline 2. PI & 6.75 & 3.80 & 3.85 & 5.90 & 5.08 & 2.68 & 1.98 & 5.97 & 4.11 & 38 & 8.06 & 7.91 & 8.68 & 9.43 & $\sqrt{15}$ & 4.43 & 3.99 & 3.63 & 4.52 & 94 \\
\hline 3. $S M$ & 3.99 & 3.86 & 3.78 & 6.48 & 4.53 & 3.99 & 5.39 & 2.06 & 10.58 & $\frac{1}{5.50}$ & 8.83 & 8.35 & 8.24 & 10.31 & 8.93 & 10.41 & 6.03 & 2.93 & 5.25 & 6.16 \\
\hline 4. CEM & 6.86 & 7.02 & 6.99 & 3.31 & 6.04 & 6.41 & 5.09 & 4.68 & 3.64 & 4.96 & 8.54 & 8.56 & 8.66 & 8.79 & 8.63 & 7.75 & 7.43 & 6.82 & 5.88 & 6.97 \\
\hline \multirow[t]{2}{*}{ 5. Menn } & 5.39 & 4.64 & 4.60 & 5.51 & 5.03 & 4.52 & 4.52 & 4.55 & 6.79 & 5.10 & 8.40 & 8.27 & 8.49 & 9.64 & 8.70 & 7.17 & 5.94 & 4.94 & 5.58 & 5.91 \\
\hline & \multicolumn{10}{|c|}{ Housing } & \multicolumn{10}{|c|}{ Durables } \\
\hline 6. SI & 6.34 & 6.98 & 7.48 & 9.97 & 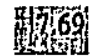 & 4.91 & 5.52 & 5.84 & 5.68 & 45 & 8.65 & 8.82 & 9.46 & 10.62 & 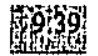 & 9.24 & 10.00 & 11.07 & 9.64 & 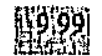 \\
\hline 7. PI & 29.88 & 6.09 & 9.52 & 13.44 & 14.73 & 26.90 & 2.74 & 8.83 & 6.34 & 11.20 & 22.96 & 7.90 & 11.59 & 12.97 & 13.85 & 16.55 & 4.98 & 12.96 & 14.72 & 12.30 \\
\hline 8. $5 M$ & 6,61 & 6.98 & 7.45 & 10.65 & 7.92 & 45.79 & 16.91 & 2.75 & 18.44 & 20.97 & 10.92 & 9.28 & 9.27 & 12.37 & 10.46 & 45.88 & 23.60 & 2.98 & 33.23 & 26.42 \\
\hline 9. CEM & 11.72 & 9.21 & 7.60 & 6.63 & 8.79 & 7.38 & 10.98 & 12.92 & 3.06 & 8.58 & 11.17 & 11.50 & 11.59 & 8.54 & 10.70 & 14.06 & 13.26 & 13.43 & 7.46 & 12.05 \\
\hline \multirow[t]{2}{*}{ 10. Mean } & 13.64 & 7.32 & 8.01 & 10.17 & 9.78 & 21.24 & 9.03 & 7.58 & 8.38 & 11.56 & 13.42 & 9.38 & 10.48 & 11.12 & 11.10 & 21.43 & 12.96 & 10.11 & 16.26 & 15.19 \\
\hline & \multicolumn{10}{|c|}{ Medicine } & \multicolumn{10}{|c|}{ Transport } \\
\hline 11.51 & 15.36 & 15.41 & 15.12 & 17.63 & 15.88 & 12.05 & 12.63 & 13.02 & 15.87 & 13.39 & 9.00 & 8.95 & 9.06 & 28.29 & 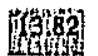 & 8.80 & 9.49 & 10.33 & 10.80 & 9.86 \\
\hline 12. $\mathrm{Pl}$ & 14.68 & 14.01 & 14.53 & 15.79 & tiphs & 9.19 & 6.41 & 5.76 & 11.10 & 8.11 & 9.97 & 8.44 & 9.62 & 33.63 & 15.41 & 9.62 & 3.96 & 3.69 & 6.67 & 5.99 \\
\hline 13. SM & 15.44 & 15.39 & 15.29 & 17.15 & 15.82 & 3.82 & 3.74 & 3.08 & 12.79 & (4) & 8.97 & 8.87 & 8.99 & 28.48 & 13.83 & 3.44 & 6.58 & 2.87 & 5.86 & 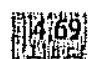 \\
\hline 14. CEM & 30.56 & 30.62 & 29.51 & 10.14 & 25.21 & 29.40 & 29.10 & 27.77 & 6.54 & 23.20 & 32.37 & 33.30 & 31.84 & 10.78 & 27.07 & 18.04 & 16.06 & 15.16 & 11.44 & 15.17 \\
\hline 15. Mean & 19.01 & 18.86 & 18.61 & 15.18 & 17.91 & 13.61 & 12.97 & 12.41 & 1158 & 12.64 & 15.08 & 14.89 & 14.88 & 25.29 & 17.53 & 9.98 & 9.02 & 8.01 & 8.69 & 8.93 \\
\hline
\end{tabular}

(Continued on next page) 
TABLE A2 (continued)

LOSSES FOR INCOME AND OWN-PRICE ELASTICITIES WHEN $\alpha=.75$

\begin{tabular}{|c|c|c|c|c|c|c|c|c|c|c|c|c|c|c|c|c|c|c|c|c|}
\hline \multirow{2}{*}{$\begin{array}{l}\text { True } \\
\text { model }\end{array}$} & \multicolumn{5}{|c|}{ Income elasticity } & \multicolumn{5}{|c|}{ Own-price elasticity } & \multicolumn{5}{|c|}{ Income elasticity } & \multicolumn{5}{|c|}{ Own-price elasticity } \\
\hline & $\begin{array}{l}\text { SI } \\
(1)\end{array}$ & $\begin{array}{l}\text { PI } \\
\text { (2) }\end{array}$ & $\begin{array}{l}S M \\
(3)\end{array}$ & $\begin{array}{l}\text { CEM } \\
\text { (4) }\end{array}$ & $\begin{array}{c}\text { Mean } \\
\text { (5) }\end{array}$ & $\begin{array}{l}\text { SI } \\
(6)\end{array}$ & $\begin{array}{l}\text { PI } \\
\text { (7) }\end{array}$ & $\begin{array}{l}S M \\
(8)\end{array}$ & $\begin{array}{c}\text { CEM } \\
(9)\end{array}$ & $\begin{array}{c}\text { Mean } \\
(10)\end{array}$ & $\begin{array}{c}\text { SI } \\
\text { (11) }\end{array}$ & $\begin{array}{l}\text { PI } \\
\text { (12) }\end{array}$ & $\begin{array}{l}\text { SM } \\
\text { (13) }\end{array}$ & $\begin{array}{l}\text { CEM } \\
(14)\end{array}$ & $\begin{array}{c}\text { Mean } \\
(15)\end{array}$ & $\begin{array}{c}\text { SI } \\
(16)\end{array}$ & $\begin{array}{c}\text { PI } \\
(17)\end{array}$ & $\begin{array}{l}S M \\
(18)\end{array}$ & $\begin{array}{l}\text { CEM } \\
(19)\end{array}$ & $\begin{array}{c}\text { Menn } \\
(20)\end{array}$ \\
\hline & \multicolumn{10}{|c|}{ Recreation } & \multicolumn{10}{|c|}{ Other } \\
\hline 16. SI & 8.23 & 8.42 & 8.56 & 11.89 & 9.28 & 7.51 & 7.61 & 7.62 & 7.75 & 7.62 & 15.66 & 15.89 & 15.91 & 42.56 & 22.50 & 20.85 & 24.91 & 25.03 & 104.34 & 43.78 \\
\hline 17. PI & 10.13 & 8.05 & 8.49 & 9.64 & mépi & 6.10 & 4.32 & 6.56 & 11.68 & 7.16 & 20.83 & 14.41 & 14.96 & 54.59 & 26.19 & 31.35 & 6.77 & 6.43 & 28.30 & 18.21 \\
\hline 18. SM & B. 38 & 8.43 & 8.47 & 11.33 & 9.15 & 8.96 & 9.06 & 3.00 & 3.61 & 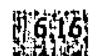 & 16.03 & 15,62 & 15.73 & 44.34 & 22.93 & 43.40 & 7.45 & 2.96 & 5.26 & 5147 \\
\hline 19. CEM & 11.53 & 11.40 & 11.54 & 7.20 & 10.42 & 10.08 & 11.50 & 11.64 & 5.77 & 9.75 & 20.19 & 19.95 & 19.80 & 9.44 & 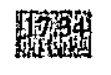 & 62.06 & 51.24 & 51.44 & 11.56 & 44.07 \\
\hline \multirow[t]{2}{*}{ 20. Menn } & 9.57 & 9.07 & 9.26 & 10.02 & 9.48 & 8.16 & 8.12 & 7.21 & 7.20 & 7.67 & 18.18 & 16.47 & 16.60 & 37.73 & 22.24 & 39.42 & 22.59 & 21.46 & 37.36 & 30.21 \\
\hline & \multicolumn{10}{|c|}{ Mean-Unweighted } & \multicolumn{10}{|c|}{ Mean-Weighted } \\
\hline 21. SI & 9.42 & 9.58 & 9.72 & 16.18 & 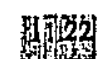 & 9.30 & 10.26 & 10.59 & 19.96 & 12.52 & 7.49 & 7.61 & 7.71 & 13.17 & 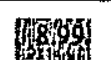 & 7.70 & 8.55 & 8.76 & 15.84 & 10.21 \\
\hline 22. PI & 13.91 & 8.82 & 9.78 & 18.72 & 12.81 & 12.67 & 4.39 & 5.95 & 10.76 & Herst & 12.65 & 7.06 & 7.89 & 15.35 & 10.74 & 10.60 & 3.52 & 5.91 & 8.41 & 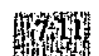 \\
\hline 23. SM & 9.69 & 9.54 & 9.65 & 16.93 & 11.45 & 20.03 & 9.62 & 2.83 & 11.51 & 11.00 & 7.66 & 7.57 & 7.66 & 13.83 & 9.18 & 17.16 & 8.94 & 2.60 & 11.39 & 10.02 \\
\hline 24. CEM & 15.56 & 15.32 & 14.72 & 8.10 & 13.43 & 18.77 & 17.70 & 17.48 & 6.92 & 15.21 & 12.70 & 12.42 & 11.82 & 6.69 & 10.91 & 14.15 & 13.23 & 13.03 & 5.90 & 11.58 \\
\hline 25. Mean & 12.15 & 10.81 & 10.97 & 14.98 & 12.23 & 15.19 & 10.49 & 9.21 & 12.29 & 11.80 & 10.12 & 8.66 & 8.77 & 12.26 & 9.95 & 12.40 & 8.56 & 7.58 & 10.38 & 9.73 \\
\hline
\end{tabular}

See notes to Table 11 . 


\section{REFERENCES}

Ahmad, E. and N. Stern (1984). "The Theory of Reform and Indian Indirect Taxes." Journal of Public Economics 25: 259-98.

and N. Stern (1987). "Alternative Sources of Government Revenue." In D. Newbery and N. Stern (eds) The Theory of Taxation for Developing Countries. Oxford: Oxford University Press. Pp. 281-332.

Barnett, W. A. (1979). "Theoretical Foundations for the Rotterdam Model." Review of Economic Studies 46: 109-30.

(1981). Consumer Demand and Labour Supply. Amsterdam: North-Holland.

Barten, A. P. (1964). "Consumer Demand Functions Under Conditions of Almost Additive Preferences." Econometrica 32: 1-38.

(1993). "Consumer Allocation Models: Choice of Functional Form." Empirical Economics 18: 129-58.

and H. Theil (1964). "Simultaneous Estimation of a Complete System of Demand Equations." Report 6405. Netherlands School of Economics, Econometric Institute.

Byron, R. P. (1984). "On the Flexibility of the Rotterdam Model." European Economic Review 24: 273-83.

Chen, D. L. (1993). World Consumption Economics. Unpublished PhD Dissertation. Economic Research Centre, Department of Economics, University of Western Australia.

(1995). "Undersized Samples and Demand Analysis." Chapter 5 in E. A. Selvanathan and K. W. Clements (eds) Recent Development in Applied Demand Analysis: Alcohol, Advertising and Global Consumption. Berlin/Heidelberg: Springer-Verlag. Pp. 191-220.

Christensen, L. R., D. W. Jorgenson and L. L. Lau (1975). "Transcendental Logarithmic Utility Functions." American Economic Review 65: 367-83.

Clements, K. W. and S. Selvanathan (1994). "Understanding Consumption Patterns." Empirical Economics 19: 69-110.

Deaton, A. (1975). Models and Projections of Demand in Post-War Britain. London: Chapman and Hall.

and J. Muellbauer (1980). "An Almost Ideal Demand System." American Economic Review 70: 312-26. 
de Boer, P. M. C. and R. Harkema (1986). "Maximum Likelihood Estimation of Sum-Constrained Linear Models with Insufficient Observations." Economics Letters 20: 325-29.

Decoster, A. and E. Schokkaert (1989). "Equity and Efficiency of a Reform of Belgian Indirect Taxes." Recherches Economiques de Louvain 55: 155-76.

and E. Schokkaert (1990). "Tax Reform Results with Different Demand Systems." Journal of Public Economics 41: 277-96.

Diewert, W. E. (1971). "An Application of the Shephard Duality Theorem: A Generalised Leontief Production Function." Journal of Political Economics 79: 481-507.

Efron, B. (1979). "Bootstrap Methods: Another Look at the Jackknife." Annals of Statistics 7: 1-26.

Howe, H., R. A. Pollak and T. L. Wales (1979). "Theory and Time-Series Estimation of the Quadratic Expenditure System." Econometrica 47: 1231-47.

Keller, W. J. (1984). "Some Simple but Flexible Differential Consumer Demand Systems." Economics Letters 16: 77-82.

and J. van Driel (1985). "Differential Consumer Demand Systems." European Economic Review 27: 375-90.

Kiefer, N. M. and J. G. MacKinnon (1976). "Small-Sample Properties of Demand System Estimates." In S. M. Goldfeld and R. E. Quandt (eds) Studies in Nonlinear Estimation. Cambridge, Mass: Ballinger. Pp. 181-210.

Madden, D. (1996). "Marginal Tax Reform and the Specification of Consumer Demand Systems." Oxford Economic Papers 48: 556-67.

Neves, P. D. (1994). "A Class of Differential Demand Systems." Economics Letters 44: 83-86.

Scales, B., B. Croser and J. Freebairn (1995). Wine-Grape and Wine Industry in Australia. Canberra: AGPS.

Selvanathan, E. A. (1985). "An Even Simpler Differential Demand System." Economics Letters 19: 343-47.

(1991). 'Further Results on the Aggregation of Differential Demand Equations." Review of Financial Studies 58: 799-805. 
Selvanathan, E. A. (1995). "Aggregation and Consumer Demand." Chapter 10 in E. A. Selvanathan and K. W. Clements (eds) Recent Developments in Applied Demand Analysis: Alcohol, Advertising and Global Consumption. Berlin/Heidelberg: Springer-Verlag. Pp. 359-90.

Selvanathan, S. (1987). "A Monte Carlo Test of Preference Independence." Economics Letters 25: 259-61.

(1991). A System-Wide Analysis of International Consumption Patterns.

Dordrecht, Boston, London: Kluwer Academic Publishers.

(1993). "The Reliability of ML Estimators of Systems of Demand Equations: Evidence from 18 Countries." Review of Economics and Statistics 73: 338-46.

Stone, R. (1954). "Linear Expenditure Systems and Demand Analysis: An Application to the Pattern of British Demand." Economic Joumal 64: 511-27.

Taylor, T. G., J. S. Shonkwiler and H. Theil (1986). "Monte Carlo and Bootstrap Testing of Demand Homogeneity." Economics Letters 20: 55-57.

Theil, H. (1965). "The Information Approach to Demand Analysis." Econometrica 33: $67-87$.

(1971). Principles of Econometrics. New York: John Wiley and Sons.

(1975/76). Theory and Measurement of Consumer Demand. Two Volumes. Amsterdam: North-Holland.

(1987). "The Econometrics of Demand Systems." Chapter 3 in H. Theil and K. W. Clements Applied Demand Analysis: Results from System-Wide Approaches. Cambridge, Mass.: Ballinger. Pp. 101-62.

and K. W. Clements (1987). Applied Demand Analysis: Results from System-Wide Approaches. Cambridge, Mass.: Ballinger.

, J. S. Shonkwiler and T. G. Taylor (1985). "A Monte Carlo Test of Slutsky Symmetry." Economics Letters 19: 331-32.

Wales, T. J. (1971). "A Generalised Linear Expenditure Model of the Demand for Nondurable Goods in Canada." Canadian Journal of Economics 4: 1471-84.

(1977). "On the Flexibility of Flexible Functional Forms." Journal of Econometrics 5: 183-93.

Working, H. (1943). "Statistical Laws of Family Expenditure." Journal of the American Statistical Association 38: 43-56. 